\title{
Gaussian approximation of maxima of Wiener functionals and its application to high-frequency data
}

\author{
Yuta Koike ${ }^{*+\$ s}$
}

February 7, 2019

\begin{abstract}
This paper establishes an upper bound for the Kolmogorov distance between the maximum of a highdimensional vector of smooth Wiener functionals and the maximum of a Gaussian random vector. As a special case, we show that the maximum of multiple Wiener-Itô integrals with common orders is well-approximated by its Gaussian analog in terms of the Kolmogorov distance if their covariance matrices are close to each other and the maximum of the fourth cumulants of the multiple Wiener-Itô integrals is close to zero. This may be viewed as a new kind of fourth moment phenomenon, which has attracted considerable attention in the recent studies of probability. This type of Gaussian approximation result has many potential applications to statistics. To illustrate this point, we present two statistical applications in high-frequency financial econometrics: One is the hypothesis testing problem for the absence of lead-lag effects and the other is the construction of uniform confidence bands for spot volatility.
\end{abstract}

Keywords: Bootstrap; Fourth moment phenomenon; Malliavin calculus; Maximum; Stein's method; Uniform confidence bands.

\section{Introduction}

This study is originally motivated by the problem of testing whether there exists a (possibly) time-lagged correlation between two Brownian motions based on their high-frequency observation data. Roughly speaking, the setting considered here is described as follows. We discretely observe the following two continuous-time processes on the interval $[0, T]$ :

$$
X_{t}^{1}=x_{0}^{1}+\sigma_{1} B_{t}^{1}, \quad X_{t}^{2}=x_{0}^{2}+\sigma_{2} B_{t-\vartheta}^{2}, \quad t \in[0, T],
$$

where $x_{0}^{1}, x_{0}^{2} \in \mathbb{R}, \sigma_{1}, \sigma_{2}>0, B_{t}=\left(B_{t}^{1}, B_{t}^{2}\right)(t \in \mathbb{R})$ is a bivariate two-sided Brownian motion with correlation $\rho \in(-1,1)$ and $\vartheta \in \mathbb{R}$. For each $\nu=1,2$, the process $X^{\nu}$ is observed at the time points $0 \leq t_{0}^{\nu}<t_{1}^{\nu}<\cdots<$ $t_{n_{\nu}}^{\nu} \leq T$, hence the observation times are possibly non-synchronous. Based on the observation data $\left(X_{t_{i}^{1}}^{1}\right)_{i=0}^{n_{1}}$ and $\left(X_{t_{j}^{2}}^{2}\right)_{j=0}^{n_{2}}$, we aim at solving the following statistical hypothesis testing problem:

$$
H_{0}: \rho=0 \quad \text { vs } \quad H_{1}: \rho \neq 0 .
$$

${ }^{*}$ Mathematics and Informatics Center and Graduate School of Mathematical Sciences, The University of Tokyo, 3-8-1 Komaba, Meguroku, Tokyo 153-8914 Japan

${ }^{\dagger}$ Department of Business Administration, Graduate School of Social Sciences, Tokyo Metropolitan University, Marunouchi Eiraku Bldg. 18F, 1-4-1 Marunouchi, Chiyoda-ku, Tokyo 100-0005 Japan

${ }^{\ddagger}$ The Institute of Statistical Mathematics, 10-3 Midori-cho, Tachikawa, Tokyo 190-8562, Japan

${ }^{\S}$ CREST, Japan Science and Technology Agency 
Model (1.1) was introduced in Hoffmann et al. [30] (as a more general one) to model lead-lag effects in highfrequency financial data (see also [56]). In [30] the problem of estimating the time-lag parameter $\vartheta$ is considered. To estimate $\vartheta$, Hoffmann et al. [30] have introduced the following contrast function:

$$
U_{n}(\theta)=\sum_{i, j}\left(X_{t_{i}^{1}}^{1}-X_{t_{i-1}^{1}}^{1}\right)\left(X_{t_{j}^{2}}^{2}-X_{t_{j-1}^{2}}^{2}\right) 1_{\left\{\left(t_{i-1}^{1}, t_{i}^{1}\right] \cap\left(t_{j-1}^{2}-\theta, t_{j}^{2}-\theta\right] \neq \emptyset\right\}} .
$$

$U_{n}(\theta)$ could be considered as the (sample) cross-covariance function between the returns of $X^{1}$ and $X^{2}$ at the lag $\theta$ computed by Hayashi and Yoshida [27]'s method. Hoffmann et al. [30] have shown that

$$
\widehat{\vartheta}_{n}=\arg \max _{\theta \in \mathcal{G}_{n}}\left|U_{n}(\theta)\right|
$$

is a consistent estimator for $\vartheta$ under some regularity conditions while one appropriately takes the finite set $\mathcal{G}_{n}$ as long as $\rho \neq 0$. The condition $\rho \neq 0$ is necessary because it is clearly impossible to identify the parameter $\vartheta$ if $\rho=0$. Therefore, unless we can believe $\rho \neq 0$ due to some external information, we need to reject the null hypothesis in the above testing problem before we carry out estimation of $\vartheta$. A natural approach to solve testing problem (1.2) is to reject the null hypothesis if the value of $\max _{\theta \in \mathcal{G}_{n}}\left|U_{n}(\theta)\right|$ is too large. To implement this idea precisely, we need to derive or approximate the distribution of $\max _{\theta \in \mathcal{G}_{n}}\left|U_{n}(\theta)\right|$ under the null hypothesis $H_{0}$. One main purpose of this paper is to give an answer to this problem. More generally, we consider the problem of approximating the distributions of maximum-type statistics appearing in high-frequency financial econometrics. Indeed, we encounter such statistics in many problems of high-frequency financial econometrics, e.g. construction of uniform confidence bands for spot volatility and other time-varying characteristics, family-wise error rate control for testing at many time points (cf. [3, 18]), change point analysis of volatility (cf. [6]), testing the absence of jumps (cf. [41, 54]) and so on.

From a mathematical point of view, this paper is built on two recent studies developed in different areas. The first one is the seminal work of Chernozhukov, Chetverikov and Kato [11, 13, 15, 16] which we call the Chernozhukov-Chetverikov-Kato theory, or the CCK theory for short. One main conclusion from the CCK theory is a bound for the Kolmogorov distance between the distributions of the maximum of a (high-dimensional) random vector and that of a Gaussian vector, which has an apparent connection to our purpose. However, their result is not directly applicable to our problem because their target random vector is a sum of independent random vectors $[11,13,16]$ or Gaussian $[15,16]$. In fact, one of our main target random vectors, $\left(U_{n}(\theta)\right)_{\theta \in \mathcal{G}_{n}}$, is a sum of dependent random vectors even under the null hypothesis where the dependence is caused by the non-synchronicity of the observation times. Although there are several extensions of the CCK theory to a sum of dependent random vectors (see e.g. $[9,10,14,62,63]$ ), it still seems difficult to apply such a result to our problem because the nonsynchronicity causes a quite complex, "non-stationary", dependence structure. In this aspect this paper aims at extending the CCK theory suitably to our purpose, and our results indeed generalize several results of [15]. In particular, our results do not require that the target random vector should be written as a sum of random vectors and give a simpler bound than those of the preceding studies listed above.

It turns out that in the CCK theory the independence/Gaussianity assumption on the target vector is crucial for the application of Stein's method. ${ }^{1}$ In other words, we can naturally extend the CCK theory to a case without independence as long as Stein's method is effectively applicable. This viewpoint leads us to using another important

\footnotetext{
${ }^{1}$ The independence assumption also plays a role in deriving maximal moment inequalities, but this issue may be considered separately.
} 
theory for this work, Malliavin calculus, in our problem. In fact, starting from the seminal work of Nourdin and Peccati [46], the recent studies show that "Stein's method and Malliavin calculus fit together admirably well" (page 3 of Nourdin [45]). This paper shows that this statement continues to hold true in the application to the CCK theory. Our application of Malliavin calculus is based on a multivariate extension of the ideas from [46], which is established in [51] (see also [47]). We refer to the monograph [48] for more information about this subject.

After developing the main Gaussian approximation results, we turn to the original problem of statistical applications in high-frequency data. In this paper we demonstrate two applications: One is testing the absence of lead-lag effects and the other is constructing uniform confidence bands for spot volatility. We have already explained the background of the former problem in the above, so we briefly discuss the latter one. Estimation of spot volatility is one of major topics in high-frequency financial econometrics (see Chapter 8 of [1] and references therein). There are quite a few articles concerning construction of pointwise confidence bands for spot volatility; see e.g. [2, 38, 42, 44]. In contrast, only a few results are available on the behavior of uniform errors in spot volatility estimation: Kristensen [38] and Kanaya and Kristensen [34] give uniform convergence rates for kerneltype spot volatility estimators, while Fan and Wang [24] consider a Gumbel approximation for the distribution of uniform errors of kernel-type spot volatility estimators. Besides, Sabel [57] implements multiscale inference for spot volatility via KMT construction. This paper contributes this relatively undeveloped areas by providing a new approach to construct uniform confidence bands for spot volatility in the spirit of the CCK theory: Construction of uniform confidence bands is a typical application of the CCK theory, cf. [12, 35, 36].

In the first application, the Gaussian approximation itself is still statistically infeasible because the covariance structure of the objective statistics is unknown. For this reason we also develop a wild (or multiplier) bootstrap procedure to approximate the quantiles of the error distribution of the test statistic, which is the approach taken in the CCK theory. The Gaussian approximation result serves as validating such a bootstrap procedure.

The remainder of this paper is organized as follows. Section 2 presents the main Gaussian approximation results obtained in this study. In Section 3 we derive Gaussian approximation results for maxima of random symmetric quadratic forms as an application of the main results. We present two statistical applications of our results in high-frequency financial econometrics in Section 4. We especially propose a testing procedure for (1.2). The finite sample performance of this testing procedure is illustrated in Section 5. We put most technical parts of the paper on the Appendix: Appendix A collects the preliminary definitions and results used in Appendix B, which contains proofs of all the results presented in the main text of the paper.

\section{Notation}

Throughout the paper, $\mathfrak{C}=(\mathfrak{C}(i, j))_{1 \leq i, j \leq d}$ denotes a $d \times d$ nonnegative definite symmetric matrix, and $Z=$ $\left(Z_{1}, \ldots, Z_{d}\right)^{\top}$ denotes a $d$-dimensional centered Gaussian random vector with covariance matrix $\mathfrak{C}$. For a vector $x=\left(x_{1}, \ldots, x_{d}\right)^{\top} \in \mathbb{R}^{d}$, we write $x_{\vee}=\max _{1 \leq j \leq d} x_{j}$. For any $\varepsilon>0$ and any subset $A$ of $\mathbb{R}$, we write $A^{\varepsilon}=\{x \in \mathbb{R}:|x-y| \leq \varepsilon$ for some $y \in A\}$. For a real-valued function $f$ defined on an interval $I \subset \mathbb{R}$ and $\eta>0$, we write $\|f\|_{\infty}=\sup \{|f(x)|: x \in I\}$ and $w(f ; \eta)=\sup \{|f(s)-f(t)|: s, t \in I,|s-t| \leq \eta\}$. For a random variable $\xi$ and $p \geq 1$, we write $\|\xi\|_{p}=\left\{E\left[|\xi|^{p}\right]\right\}^{1 / p}$. For a matrix $A$, we denote by $\|A\|_{\text {sp }}$ and $\|A\|_{F}$ its spectral norm and Frobenius norm, respectively.

Finally, we enumerate the notation from Malliavin calculus which are necessary to state our main results. We refer to $[32,48,52]$ for a detailed description of Malliavin calculus. Also, see Section A.1 of Appendix A for a 
concise overview of the notions from Malliavin calculus used in this paper.

- Throughout the paper, $H$ denotes a real separable Hilbert space. The inner product and the norm of $H$ are denoted by $\langle\cdot, \cdot\rangle_{H}$ and $\|\cdot\|_{H}$, respectively.

- We assume that an isonormal Gaussian process $W=(W(h))_{h \in H}$ over $H$ defined on a probability space $(\Omega, \mathcal{F}, P)$ is given. We denote by $L^{2}(W)$ the space $L^{2}(\Omega, \sigma(W), P)$ for short.

- For a non-negative integer $q, H^{\otimes q}$ and $H^{\odot q}$ denote the $q$ th tensor power and $q$ th symmetric tensor power, respectively.

- For an element $f \in H^{\odot q}$ we denote by $I_{q}(f)$ the $q$ th multiple Wiener Itô integral of $f$.

- For any real number $p \geq 1$ and any integer $k \geq 1, \mathbb{D}_{k, p}$ denotes the stochastic Sobolev space of random variables which are $k$ times differentiable in the Malliavin sense and the derivatives up to order $k$ have finite moments of order $p$. If $F \in \mathbb{D}_{k, p}$, we denote by $D^{k} F$ the $k$ th Malliavin derivative of $F$. We write $D F$ instead of $D^{1} F$ for short.

- $L$ denotes the Ornstein-Uhlenbeck operator. Also, the pseudo inverse of $L$ is denoted by $L^{-1}$.

\section{Main results}

Throughout this section, $F=\left(F_{1}, \ldots, F_{d}\right)^{\top}$ denotes a $d$-dimensional random vector such that $F_{j} \in \mathbb{D}_{1,2}$ and $E\left[F_{j}\right]=0$ for all $j=1, \ldots, d$. For each $\beta>0$, we define the function $\Phi_{\beta}: \mathbb{R}^{d} \rightarrow \mathbb{R}$ by

$$
\Phi_{\beta}(x)=\beta^{-1} \log \left(\sum_{j=1}^{d} e^{\beta x_{j}}\right) \quad\left(x=\left(x_{1}, \ldots, x_{d}\right)^{\top} \in \mathbb{R}^{d}\right) .
$$

Eq.(1) from [15] states that

$$
0 \leq \Phi_{\beta}(x)-x_{\vee} \leq \beta^{-1} \log d
$$

for any $x \in \mathbb{R}^{d}$.

We first give a generalization of Theorem 1 from [15] as follows:

Proposition 2.1. Let $g: \mathbb{R} \rightarrow \mathbb{R}$ be a $C^{2}$ function with bounded first and second derivatives. Then, for any $\beta>0$ we have

$$
\left|E\left[g\left(\Phi_{\beta}(F)\right)\right]-E\left[g\left(\Phi_{\beta}(Z)\right)\right]\right| \leq\left(\left\|g^{\prime \prime}\right\|_{\infty} / 2+\beta\left\|g^{\prime}\right\|_{\infty}\right) \Delta
$$

where

$$
\Delta=E\left[\max _{1 \leq i, j \leq d}\left|\mathfrak{C}(i, j)-\left\langle D F_{i},-D L^{-1} F_{j}\right\rangle_{H}\right|\right]
$$

In particular, it holds that

$$
\left|E\left[g\left(F_{\vee}\right)\right]-E\left[g\left(Z_{\vee}\right)\right]\right| \leq\left(\left\|g^{\prime \prime}\right\|_{\infty} / 2+\beta\left\|g^{\prime}\right\|_{\infty}\right) \Delta+2 \beta^{-1}\left\|g^{\prime}\right\|_{\infty} \log d
$$

Remark 2.1. We can indeed derive Theorem 1 of [15] from Proposition 2.1 in the following way. Suppose that the law of $F$ is the $d$-dimensional normal distribution with mean 0 and covariance matrix $\Sigma=(\Sigma(i, j))_{1 \leq i, j \leq d}$. Without loss of generality, we may assume that $F$ is expressed as $F=\Sigma^{1 / 2} G$ with $G$ being a $d$-dimensional standard Gaussian vector. Then we can define the isonormal Gaussian process $W$ over $H:=\mathbb{R}^{d}$ by $W(h)=h^{\top} G$, $h \in H$ (cf. Example 2.1.3 of [48]), and we have $F_{i}=\sum_{j=1}^{d} \gamma_{i j} W\left(e_{j}\right)$ for every $i=1, \ldots, d$, where $\gamma_{i j}$ denotes 
the $(i, j)$-th component of the matrix $\Sigma^{1 / 2}$ and $\left(e_{1}, \ldots, e_{d}\right)$ denotes the canonical basis of $\mathbb{R}^{d}$. In this case it holds that

$$
\left\langle D F_{i},-D L^{-1} F_{j}\right\rangle_{H}=\sum_{k, l=1}^{d} \gamma_{i k} \gamma_{j l}\left\langle e_{k}, e_{l}\right\rangle_{H}=\sum_{k=1}^{d} \gamma_{i k} \gamma_{j k}=\Sigma(i, j),
$$

hence we obtain the conclusion of Theorem 1 from [15].

Proposition 2.1 and some elementary approximation arguments lead the following useful lemma:

Lemma 2.1. There is a universal constant $C>0$ such that

$$
P\left(F_{\vee} \in A\right) \leq P\left(Z_{\vee} \in A^{5 \varepsilon}\right)+C \varepsilon^{-2}(\log d) \Delta
$$

for any Borel set $A$ of $\mathbb{R}$ and any $\varepsilon>0$.

Remark 2.2. Lemma 2.1 is useful when we derive a Gaussian approximation for the supremum of statistics indexed by an infinite set (see Proposition 4.3 and its proof). In fact, Lemma 2.1 can be considered as a counterpart of Theorem 3.1 from [16], which is used to derive their Gaussian approximation results for suprema of empirical processes. An advantage of Lemma 2.1 over Theorem 3.1 from [16] is that the second term of the estimate is proportional to $\varepsilon^{-2}$ in Lemma 2.1, while it is proportional to $\varepsilon^{-3}$ in Theorem 3.1 from [16]. This difference generally leads a weaker condition and a better convergence rate in Gaussian approximation; see Remark 4.8 for details.

Combining Lemma 2.1 with several technical tools developed in the CCK theory, we obtain the following main result of this paper, which can be considered as a generalization of Theorem 2 from [15]:

Theorem 2.1. (a) Suppose that $d \geq 2$ and there are constants $\underline{\sigma}, \bar{\sigma}>0$ such that $\underline{\sigma}^{2} \leq \mathfrak{C}(j, j) \leq \bar{\sigma}^{2}$ for all $j=1, \ldots, d$. Set $a_{d}=E\left[\max _{1 \leq j \leq d}\left(Z_{j} / \sqrt{\mathfrak{C}(j, j)}\right)\right]$. Then

$$
\sup _{x \in \mathbb{R}}\left|P\left(F_{\vee} \leq x\right)-P\left(Z_{\vee} \leq x\right)\right| \leq C \Delta^{1 / 3}\left\{1 \vee a_{d}^{2} \vee \log (1 / \Delta)\right\}^{1 / 3}(\log d)^{1 / 3},
$$

where $C>0$ depends only on $\underline{\sigma}$ and $\bar{\sigma}$ (the right side is understood to be 0 if $\Delta=0$ ).

(b) Suppose that $d \geq 2$ and there is a constant $b>0$ such that $\mathfrak{C}(j, j) \geq b$ for all $j=1, \ldots, d$. Then

$$
\sup _{x \in \mathbb{R}}\left|P\left(F_{\vee} \leq x\right)-P\left(Z_{\vee} \leq x\right)\right| \leq C^{\prime} \Delta^{1 / 3}(\log d)^{2 / 3},
$$

where $C^{\prime}>0$ depends only on $b$.

Since we have $\max _{1 \leq j \leq d}\left|x_{j}\right|=\max \left\{\max _{1 \leq j \leq d} x_{j}, \max _{1 \leq j \leq d}\left(-x_{j}\right)\right\}$ for any real numbers $x_{1}, \ldots, x_{d}$, we obtain the following result as a direct consequence of Theorem 2.1:

Corollary 2.1. Under the assumptions of Theorem 2.1(b), we have

$$
\sup _{x \in \mathbb{R}}\left|P\left(\max _{1 \leq j \leq d}\left|F_{j}\right| \leq x\right)-P\left(\max _{1 \leq j \leq d}\left|Z_{j}\right| \leq x\right)\right| \leq C^{\prime} \Delta^{1 / 3}(\log d)^{2 / 3}
$$

where $C^{\prime}>0$ depends only on $b$.

In order to make Theorem 2.1 and Corollary 2.1 useful, we need a reasonable bound for the quantity $\Delta$. When the random vector $F$ consists of multiple Wiener-Itô integrals with common order, we have the following useful bound for $\Delta$ : 
Lemma 2.2. Let $q \geq 2$ be an integer and suppose that $F_{j}=I_{q}\left(f_{j}\right)$ for some $f_{j} \in H^{\odot q}$ for $j=1, \ldots, d$. Then we have

$$
\Delta \leq \max _{1 \leq i, j \leq d}\left|\mathfrak{C}(i, j)-E\left[F_{i} F_{j}\right]\right|+C_{q} \log ^{q-1}\left(2 d^{2}-1+e^{q-2}\right) \max _{1 \leq k \leq d} \sqrt{E\left[F_{k}^{4}\right]-3 E\left[F_{k}^{2}\right]^{2}},
$$

where $C_{q}>0$ depends only on $q$.

Remark 2.3. Lemma 2.2 implies that, in order to bound the Kolmogorov distance between $F_{\vee}$ and $Z_{\vee}$, we only need to control the convergence rate of the covariance matrix of $F$ to that of $Z$ and the fourth cumulants of the components of $F$, as long as $F$ consists of multiple Wiener-Itô integrals with common order. This can be considered as a type of fourth moment phenomenon, which was first discovered by [53] while they derived central limit theorems for sequences of multiple Wiener-Itô integrals. For more information about the fourth moment phenomenon, we refer to [48] and references therein.

It is often involved to compute the variables $L^{-1} F_{j}$ in the case that $F_{j}$ 's are general Wiener functionals. It would be worth mentioning that we can avoid this issue if the variables $F_{j}$ are twice differentiable in the Malliavin sense and satisfy a suitable moment condition. To state such a result precisely, we make some definitions: For an $H \otimes H$ valued random variable $G$, we denote by $\|G\|_{\text {op }}$ the operator norm of the (random) operator $H \ni h \mapsto\langle h, G\rangle_{H} \in$ $H$. Also, we say that a random variable $Y$ is sub-Gaussian relative to the scale a $>0$ if $E\left[e^{\lambda Y}\right] \leq \exp \left(\lambda^{2} a^{2} / 2\right)$ for all $\lambda \in \mathbb{R}$.

Lemma 2.3. If $F_{1}, \ldots, F_{d} \in \mathbb{D}_{2,4 p}$ for a positive integer $p$, we have

$$
\Delta \leq \max _{1 \leq i, j \leq d}\left|\mathfrak{C}(i, j)-E\left[F_{i} F_{j}\right]\right|+d^{1 / p} \sqrt{2 p-1} \cdot \frac{3}{2}\left(\max _{1 \leq i \leq d}\|\| D^{2} F_{i}\left\|_{o p}\right\|_{4 p}\right)\left(\max _{1 \leq j \leq d}\|\| D F_{j}\left\|_{H}\right\|_{4 p}\right) .
$$

Moreover, if there is a constant $a>0$ such that both the variables $\left\|D^{2} F_{i}\right\|_{o p}$ and $\left\|D F_{i}\right\|_{H}$ are sub-Gaussian relative to the scale a for all $i=1, \ldots, d$, we have

$$
\Delta \leq \max _{1 \leq i, j \leq d}\left|\mathfrak{C}(i, j)-E\left[F_{i} F_{j}\right]\right|+C a^{2} \log ^{3 / 2}\left(2 d^{2}-1+\sqrt{e}\right),
$$

where $C>0$ is a universal constant.

Remark 2.4. The above result (combined with Theorem 2.1) can be viewed as an analogy of the so-called secondorder Poincaré inequalities proved in Nourdin et al. [49]. Indeed, its proof is based on the lemmas proved there.

\section{Gaussian approximation of maxima of random symmetric quadratic forms}

In this section we focus on the problem of approximating the distribution of maxima of symmetric quadratic forms. The next result can be easily derived from the results in the previous section:

Theorem 3.1. For each $n \in \mathbb{N}$, let $\boldsymbol{\xi}_{n}$ be an $N_{n}$-dimensional centered Gaussian vector with covariance matrix $\Sigma_{n}=\left(\Sigma_{n}(k, l)\right)_{1 \leq k, l \leq N_{n}}$ and $d_{n} \geq 2$ be an integer. Also, for each $k=1, \ldots, d_{n}$, let $A_{n, k}$ be an $N_{n} \times N_{n}$ symmetric matrix and $Z_{n}=\left(Z_{n, 1}, \ldots, Z_{n, d_{n}}\right)^{\top}$ be an $d_{n}$-dimensional centered Gaussian vector with covariance matrix $\mathfrak{C}_{n}=\left(\mathfrak{C}_{n}(k, l)\right)_{1 \leq k, l \leq d_{n}}$. Set $F_{n, k}:=\boldsymbol{\xi}_{n}^{\top} A_{n, k} \boldsymbol{\xi}_{n}-E\left[\boldsymbol{\xi}_{n}^{\top} A_{n, k} \boldsymbol{\xi}_{n}\right]$ and suppose that the following conditions are satisfied:

(i) There is a constant $b>0$ such that $\mathfrak{C}_{n}(k, k) \geq b$ for every $n$ and every $k=1, \ldots, d_{n}$.

(ii) $\max _{1 \leq k \leq d_{n}}\left(E\left[F_{n, k}^{4}\right]-3 E\left[F_{n, k}^{2}\right]^{2}\right) \log ^{6} d_{n} \rightarrow 0$ as $n \rightarrow \infty$. 
(iii) $\max _{1 \leq k, l \leq d_{n}}\left|\mathfrak{C}_{n}(k, l)-E\left[F_{n, k} F_{n, l}\right]\right| \log ^{2} d_{n} \rightarrow 0$ as $n \rightarrow \infty$.

Then we have

$$
\sup _{x \in \mathbb{R}}\left|P\left(\max _{1 \leq k \leq d_{n}} F_{n, k} \leq x\right)-P\left(\max _{1 \leq k \leq d_{n}} Z_{n, k} \leq x\right)\right| \rightarrow 0
$$

and

$$
\sup _{x \in \mathbb{R}}\left|P\left(\max _{1 \leq k \leq d_{n}}\left|F_{n, k}\right| \leq x\right)-P\left(\max _{1 \leq k \leq d_{n}}\left|Z_{n, k}\right| \leq x\right)\right| \rightarrow 0
$$

as $n \rightarrow \infty$.

Remark 3.1. Since any symmetric Gaussian quadratic form can be written as a linear combination of independent $\chi^{2}$ random variables via eigenvalue decomposition (see e.g. Section 3.2.1 of [19]), the readers may be wondering about whether it is possible to apply the original CCK theory to derive a similar result to Theorem 3.1 using eigenvalue decomposition. This is however impossible in general because the matrices $\Sigma_{n}^{1 / 2} A_{n, 1} \Sigma_{n}^{1 / 2}, \ldots, \Sigma_{n}^{1 / 2} A_{n, d_{n}} \Sigma_{n}^{1 / 2}$ are not necessarily simultaneously diagonalizable by an orthogonal matrix, which may induce an additional crosssectional dependence after orthogonal transformation. To see this, suppose that $\Sigma_{n}$ is identity for simplicity. Then, the afore-mentioned eigenvalue decomposition argument reads as follows: For each $k=1, \ldots, d_{n}$, we take an $N_{n} \times N_{n}$ real orthogonal matrix $U_{n, k}$ such that $U_{n, k} A_{n, k} U_{n, k}^{\top}$ is diagonal, and set $\varepsilon_{n, k}=U_{n, k} \boldsymbol{\xi}_{n}$. Then the components of $\varepsilon_{n, k}$ are independent and $F_{n, k}$ can be written as a linear combination of the squared components of $\varepsilon_{n, k}$. However, for $k \neq l$, the covariance matrix of $\varepsilon_{n, k}$ and $\varepsilon_{n, l}$ is given by $U_{n, k} U_{n, l}^{\top}$, which is generally not diagonal; e.g. we have

$$
\frac{1}{\sqrt{2}}\left(\begin{array}{cc}
1 & 1 \\
1 & -1
\end{array}\right)\left(\frac{1}{\sqrt{5}}\left(\begin{array}{cc}
2 & 1 \\
1 & -2
\end{array}\right)\right)^{\top}=\frac{1}{\sqrt{10}}\left(\begin{array}{cc}
3 & -1 \\
1 & 3
\end{array}\right) .
$$

Remark 3.2. Even if the matrices $\Sigma_{n}^{1 / 2} A_{n, 1} \Sigma_{n}^{1 / 2}, \ldots, \Sigma_{n}^{1 / 2} A_{n, d_{n}} \Sigma_{n}^{1 / 2}$ are simultaneously diagonalizable, there is gain to use Theorem 3.1 instead of the original CCK theory. To see this, suppose that each $F_{n, k}$ can be written as

$$
F_{n, k}=\sum_{i=1}^{N_{n}} \lambda_{n, k}(i)\left(\eta_{i}^{2}-1\right)
$$

where $\lambda_{n, k}(1), \ldots, \lambda_{n, k}\left(N_{n}\right) \in \mathbb{R}$ and $\left(\eta_{i}\right)_{i=1}^{\infty}$ is a sequence of i.i.d. standard normal variables. In this case, if we assume that there are constants $\bar{b}, \underline{b}>0$ such that

$$
\underline{b} \leq \sum_{i=1}^{N_{n}} \lambda_{n, k}(i)^{2} \leq \bar{b}
$$

for all $n \in \mathbb{N}$ and $k=1, \ldots, d_{n}$ and that the matrix $\mathfrak{C}_{n}$ is equal to the covariance matrix of the variables $F_{n, 1}, \ldots, F_{n, d_{n}}$, Proposition 2.1 of [17] yields the convergence (3.1), provided that $B_{n}^{2} \log ^{7}\left(d_{n} N_{n}\right)=o\left(N_{n}\right)$ as $n \rightarrow \infty$, where

$$
B_{n}=\sqrt{N_{n}} \max _{1 \leq k \leq d_{n}} \max _{1 \leq i \leq N_{n}}\left|\lambda_{n, k}(i)\right| .
$$

Since we have

$$
\max _{1 \leq k \leq d_{n}}\left(E\left[F_{n, k}^{4}\right]-3 E\left[F_{n, k}^{2}\right]^{2}\right) \leq \frac{B_{n}^{2}}{N_{n}} \bar{b}
$$


the convergence (3.1) is indeed implied by $B_{n}^{2} \log ^{6} d_{n}=o\left(N_{n}\right)$ according to Theorem 3.1. In addition, the inequality (3.2) can be not tight. A cheap example is the case that

$$
\lambda_{n, k}(i)= \begin{cases}1 / N_{n}^{1 / 4} & \text { if } i=k \\ 1 / \sqrt{N_{n}} & \text { otherwise }\end{cases}
$$

In this case we have $B_{n}^{2} / N_{n}=1 / \sqrt{N_{n}}$, while it holds that

$$
\max _{1 \leq k \leq d_{n}}\left(E\left[F_{n, k}^{4}\right]-3 E\left[F_{n, k}^{2}\right]^{2}\right)=O\left(N_{n}^{-1}\right) .
$$

See also Remark 4.8 for another advantage of using our results instead of the original CCK theory.

Remark 3.3 (Discussion on the fourth moment condition (ii)).

(i) In Theorem 3.1, the number $N_{n}$ does not necessarily diverge to get the convergence $E\left[F_{n, k}^{4}\right]-3 E\left[F_{n, k}^{2}\right]^{2} \rightarrow$ 0 . This is because the variance of $\boldsymbol{\xi}_{n}$ is allowed to diverge in the setting of the theorem. To see this, suppose that $N_{n}=1, \boldsymbol{\xi}_{n}$ is a centered Gaussian variable with variance $n$, and $A_{n, k}=1 / \sqrt{2 n}$. In this case we have $F_{n, k}=\left(\boldsymbol{\xi}_{n}^{2}-n\right) / \sqrt{2 n}$ and thus $E\left[F_{n, k}^{2}\right]=1$ and $E\left[F_{n, k}^{4}\right]-3 E\left[F_{n, k}^{2}\right]^{2}=12 / n \rightarrow 0$.

(ii) If $\sup _{n \in \mathbb{N}} \max _{1 \leq k \leq d_{n}} E\left[F_{n, k}^{2}\right]<\infty$, a sufficient condition to prove the condition (ii) of Theorem 3.1 is $\max _{1 \leq k \leq d_{n}}\left\|\Sigma_{n}^{1 / 2} A_{n, k} \Sigma_{n}^{1 / 2}\right\|_{\text {sp }} \log ^{3} d_{n} \rightarrow 0$ as $n \rightarrow \infty$. This follows from the following inequality (see Eq.(11) of [19]):

$$
E\left[F_{n, k}^{4}\right]-3 E\left[F_{n, k}^{2}\right]^{2}=48 \operatorname{tr}\left[\left(\Sigma_{n}^{1 / 2} A_{n, k} \Sigma_{n}^{1 / 2}\right)^{4}\right] \leq 24\left\|\Sigma_{n}^{1 / 2} A_{n, k} \Sigma_{n}^{1 / 2}\right\|_{\mathrm{sp}}^{2} E\left[F_{n, k}^{2}\right]
$$

(note that we always have $E\left[F_{n, k}^{4}\right]-3 E\left[F_{n, k}^{2}\right]^{2} \geq 0$; see Remark 5.2.5 of [48]). In practice, it is often easier to check the condition on $\left\|\Sigma_{n}^{1 / 2} A_{n, k} \Sigma_{n}^{1 / 2}\right\|_{\text {sp }}$ than to directly check the condition on $E\left[F_{n, k}^{4}\right]-3 E\left[F_{n, k}^{2}\right]^{2}$.

(iii) The condition $E\left[F_{n, k}^{4}\right]-3 E\left[F_{n, k}^{2}\right]^{2} \rightarrow 0$ is necessary to approximate the distribution of the random variable $F_{n, k}$ by a Gaussian distribution if $\sup _{n \in \mathbb{N}} E\left[F_{n, k}^{2}\right]<\infty$ because there is a universal constant $c>0$ such that

$$
\sup _{n \in \mathbb{N}} E\left[F_{n, k}^{8}\right] \leq c \sup _{n \in \mathbb{N}} E\left[F_{n, k}^{2}\right]^{4}<\infty
$$

(see e.g. Theorem 5.10 of [32]), which implies the uniform integrability of the variables $F_{n, k}^{2}$ and $F_{n, k}^{4}$, $n=1,2, \ldots$ Actually, adopting an analogous discussion to the one from Chernozhukov et al. [17], we can easily generalize the conclusion of Theorem 3.1 to the convergence of the Kolmogorov distance between $F_{n}$ and $Z_{n}$ as follows:

$$
\sup _{x_{1}, \ldots, x_{d_{n}} \in \mathbb{R}}\left|P\left(\bigcap_{k=1}^{d_{n}}\left\{F_{n, k} \leq x_{k}\right\}\right)-P\left(\bigcap_{k=1}^{d_{n}}\left\{Z_{n, k} \leq x_{k}\right\}\right)\right| \rightarrow 0 .
$$

Therefore, if $\sup _{n \in \mathbb{N}} \max _{1 \leq k \leq d_{n}} E\left[F_{n, k}^{2}\right]<\infty$, the condition $\max _{1 \leq k \leq d_{n}}\left(E\left[F_{n, k}^{4}\right]-3 E\left[F_{n, k}^{2}\right]^{2}\right) \log ^{6} d_{n} \rightarrow$ 0 as $n \rightarrow \infty$ is indeed a necessary condition when $d_{n}$ is fixed (it is still unclear that this condition is necessary when $d_{n} \rightarrow \infty$ as $n \rightarrow \infty$, though).

In the next section we will apply Theorem 3.1 to derive a Gaussian approximation of the null distribution of the test statistic for the absence of lead-lag effects. In order to implement the test in practice, we need to compute 
quantiles of the null distribution, but it is not easy to directly compute those of the derived Gaussian analog of the test statistic because its covariance structure contains unknown quantities for statisticians. For this reason we will apply a wild bootstrap procedure to approximately compute quantiles of the null distribution. Theorem 3.1 is still applicable for ensuring the validity of such a procedure as long as Gaussian wild bootstrapping is considered, but it turns out that a wild bootstrap procedure based on another distribution performs much better in finite samples. For this reason we partially generalize Theorem 3.1 to a non-Gaussian case.

For every $n \in \mathbb{N}$, let $N_{n} \geq 1$ and $d_{n} \geq 2$ be integers and let $\Gamma_{n, k}=\left(\gamma_{n, k}(i, j)\right)_{1 \leq i, j \leq N_{n}}$ be an $N_{n} \times N_{n}$ symmetric matrix for each $k=1, \ldots, d_{n}$. We assume that $\gamma_{n, k}(i, i)=0$ for all $i=1, \ldots, N_{n}, k=1, \ldots, d_{n}$ and $n \in \mathbb{N}$. Given a sequence $\xi=\left(\xi_{i}\right)_{i=1}^{\infty}$ of random variables, we set

$$
Q_{n, k}(\xi):=\sum_{i, j=1}^{N_{n}} \gamma_{n, k}(i, j) \xi_{i} \xi_{j}, \quad k=1, \ldots, d_{n}
$$

for every $n \in \mathbb{N}$.

Let $Y=\left(Y_{i}\right)_{i=1}^{\infty}$ be a sequence of independent variables such that $E\left[Y_{i}\right]=0$ and $E\left[Y_{i}^{2}\right]=1$ for every $i$. Also, let $G=\left(G_{i}\right)_{i=1}^{\infty}$ be a sequence of independent standard Gaussian variables. For every $i \in \mathbb{N}$, we define the random variables $\left(W_{j}^{(i)}\right)_{j=1}^{\infty}$ by

$$
W_{j}^{(i)}= \begin{cases}Y_{j} & \text { if } j \leq i, \\ G_{j} & \text { if } j>i .\end{cases}
$$

Theorem 3.2. For each $n \in \mathbb{N}$, let $Z_{n}=\left(Z_{n, 1}, \ldots, Z_{n, d_{n}}\right)^{\top}$ be a $d_{n}$-dimensional centered Gaussian vector with covariance matrix $\mathfrak{C}_{n}=\left(\mathfrak{C}_{n}(k, l)\right)_{1 \leq k, l \leq d_{n}}$, and set

$$
\begin{aligned}
R_{n, 1} & =\sum_{i=1}^{N_{n}} E\left[\max _{1 \leq k \leq d_{n}}\left|\sum_{j=1}^{N_{n}} \gamma_{n, k}(i, j) W_{j}^{(i)}\right|^{3}\right]\left(E\left[\left|Y_{i}\right|^{3}\right]+E\left[\left|G_{i}\right|^{3}\right]\right), \\
R_{n, 2} & =\max _{1 \leq k, l \leq d_{n}}\left|\mathfrak{C}_{n}(k, l)-E\left[Q_{n, k}(G) Q_{n, l}(G)\right]\right|, \\
R_{n, 3} & =\max _{1 \leq k \leq d_{n}} \sqrt{E\left[Q_{n, k}(G)^{4}\right]-3 E\left[Q_{n, k}(G)^{2}\right]^{2}} .
\end{aligned}
$$

Suppose that there is a constant $b>0$ such that $\mathfrak{C}_{n}(k, k) \geq b$ for every $n$ and every $k=1, \ldots, d_{n}$. Then we have

$$
\sup _{x \in \mathbb{R}}\left|P\left(\max _{1 \leq k \leq d_{n}}\left|Q_{n, k}(Y)\right| \leq x\right)-P\left(\max _{1 \leq k \leq d_{n}}\left|Z_{n, k}\right| \leq x\right)\right| \rightarrow 0
$$

as $n \rightarrow \infty$, provided that $R_{n, 1} \log ^{\frac{7}{2}} d_{n} \vee R_{n, 2} \log ^{2} d_{n} \vee R_{n, 3} \log ^{3} d_{n} \rightarrow 0$.

Remark 3.4. The variables $W_{j}^{(i)}$ are related to the so-called Lindeberg method. In fact, our proof of Theorem 3.2 is based on the generalized Lindeberg method developed in [43, 50] (see also Chapter 11 of [48]).

Remark 3.5. There is probably room for improvement in Theorem 3.2. In particular, the truncation arguments used in the CCK theory (based on Lemma A.6 of [11]) are apparently applicable to our case, which would significantly weaken the assumptions of Theorem 3.2. On the other hand, it is less obvious whether the other techniques used in the CCK theory (especially in Chernozhukov et al. [17]) are applicable to our case or not. Their excellent argument leads a very sharp bound, but it seems crucial in their argument that the statistics considered there is a linear function of independent random variables. More precisely, to apply their argument to our case, the independence 
between the variables $U_{i}$ and $V_{i}$ appearing in the proof of Theorem 3.2 seems necessary, but this is not the case (such a structure is necessary to get an analogous estimate to Eq.(30) of [17], for example). This issue is left to future research.

Remark 3.6. Analogous quantities to $R_{n, 2}$ and $R_{n, 3}$ from Theorem 3.2 have already appeared in Theorem 3.1 and it is usually not difficult to bound them. On the other hand, as long as the third moments of $Y_{i}$ 's are uniformly bounded, the quantity $R_{n, 1}$ is bounded by the third moment of the maximum of a sum of (high-dimensional) independent random vectors, so we have many inequalities which can be used to bound it (see e.g. Chapter 14 of [7]). Here we give two examples of such inequalities. The first one only requires the uniform boundedness of the $p$-th moments of $Y_{i}$ 's for some $p \geq 3$, while the latter one is applicable when the variables $Y_{i}$ are sub-Gaussian.

\section{Lemma 3.1.}

(a) Suppose that $\sup _{i \in \mathbb{N}}\left\|Y_{i}\right\|_{p}<\infty$ for some $p \geq 3$. Then

$$
\sum_{i=1}^{N_{n}} E\left[\max _{1 \leq k \leq d_{n}}\left|\sum_{j=1}^{N_{n}} \gamma_{n, k}(i, j) W_{j}^{(i)}\right|^{3}\right] \leq 2 d_{n}^{3 / p}(p-1)^{3 / 2} \sup _{i \in \mathbb{N}}\left\|Y_{i}\right\|_{p}^{3} \sum_{i=1}^{N_{n}} \max _{1 \leq k \leq d_{n}}\left(\sum_{j=1}^{N_{n}} \gamma_{n, k}(i, j)^{2}\right)^{3 / 2}
$$

for every $n$.

(b) Suppose that there is a constant a $>0$ such that $Y_{i}$ is sub-Gaussian relative to the scale a for all $i=1,2, \ldots$ Then

$$
\sum_{i=1}^{N_{n}} E\left[\max _{1 \leq k \leq d_{n}}\left|\sum_{j=1}^{N_{n}} \gamma_{n, k}(i, j) W_{j}^{(i)}\right|^{3}\right] \leq 5^{3 / 2} a^{3} \log ^{3 / 2}\left(2 d_{n}-1+\sqrt{e}\right) \sum_{i=1}^{N_{n}} \max _{1 \leq k \leq d_{n}}\left(\sum_{j=1}^{N_{n}} \gamma_{n, k}(i, j)^{2}\right)^{3 / 2}
$$

for every $n$.

Using the above lemma, we obtain a useful criterion to check the conditions appearing in Theorem 3.2 in terms of the so-called influence indices: Given a symmetric matrix $\Gamma=(\gamma(i, j))_{1 \leq i, j \leq N}$, the influence of the variable $i$ of $\Gamma$ is defined by

$$
\operatorname{Inf}_{i}(\Gamma)=\sum_{j=1}^{N} \gamma(i, j)^{2}
$$

for $i=1, \ldots, N$. The influence indices play an important role in studies of the central limit theorem for random quadratic forms (and homogeneous sums more generally); see [25, 43, 50] for example.

Corollary 3.1. Suppose that there is a constant $a>0$ such that $Y_{i}$ is sub-Gaussian relative to the scale a for all $i=1,2, \ldots$ Then the convergences $R_{n, 1} \log ^{\frac{7}{2}} d_{n} \rightarrow 0$ and $R_{n, 3} \log ^{3} d_{n} \rightarrow 0$ are implied by the following condition:

$$
\left(\log d_{n}\right)^{6} \max _{1 \leq k \leq d_{n}} \operatorname{tr}\left(\Gamma_{n, k}^{4}\right)+\left(\log d_{n}\right)^{5}\left(\max _{1 \leq i \leq N_{n}} \sqrt{\Lambda_{n, i}}\right) \sum_{i=1}^{N_{n}} \Lambda_{n, i} \rightarrow 0 \quad \text { as } n \rightarrow \infty
$$

where

$$
\Lambda_{n, i}=\max _{1 \leq k \leq d_{n}} \operatorname{Inf}_{i}\left(\Gamma_{n, k}\right), \quad i=1, \ldots, N_{n}
$$


Remark 3.7 (Implication of the condition (3.3)). Let us consider the case that there is a symmetric matrix $\bar{\Gamma}_{n}=$ $\left(\bar{\gamma}_{n}(i, j)\right)_{1 \leq i, j \leq N_{n}}$ such that $\operatorname{Inf}_{i}\left(\bar{\Gamma}_{n}\right)=\Lambda_{n, i}$ for all $i=1, \ldots, N_{n}$. Namely, the influence indices of the matrices $\Gamma_{n, 1}, \ldots, \Gamma_{n, d_{n}}$ are dominated by that of the matrix $\bar{\Gamma}_{n}$. In this case the condition (3.3) reads as

$$
\left(\log d_{n}\right)^{6} \max _{1 \leq k \leq d_{n}} \operatorname{tr}\left(\Gamma_{n, k}^{4}\right)+\left(\log d_{n}\right)^{10}\left\|\bar{\Gamma}_{n}\right\|_{F}^{4} \max _{1 \leq i \leq N_{n}} \operatorname{Inf}_{i}\left(\bar{\Gamma}_{n}\right) \rightarrow 0 \quad \text { as } n \rightarrow \infty .
$$

The quantity $\left\|\bar{\Gamma}_{n}\right\|_{F}^{2}$ is the variance of the quadratic form

$$
\bar{Q}_{n}(Y)=\sum_{i, j=1}^{N_{n}} \bar{\gamma}_{n}(i, j) Y_{i} Y_{j} .
$$

Therefore, it would be natural to assume $\sup _{n \in \mathbb{N}}\left\|\bar{\Gamma}_{n}\right\|_{F}^{2}<\infty$. Moreover, in many cases it is reasonable to expect $\max _{1 \leq i \leq d_{n}} \operatorname{Inf}_{i}\left(\bar{\Gamma}_{n}\right)=O\left(N_{n}^{-1}\right)$ because we have by definition

$$
\max _{1 \leq i \leq d_{n}} \operatorname{Inf}_{i}\left(\bar{\Gamma}_{n}\right) \leq\left\|\bar{\Gamma}_{n}\right\|_{\mathrm{sp}}^{2}
$$

According to [25], $\left\|\bar{\Gamma}_{n}\right\|_{\text {sp }}$ gives an optimal convergence rate for the Kolmogorov distance between

$$
\bar{Q}_{n}(Y) / \sqrt{\operatorname{Var}\left[\bar{Q}_{n}(Y)\right]}
$$

and a standard Gaussian variable, hence it seems reasonable to expect $\left\|\bar{\Gamma}_{n}\right\|_{\mathrm{sp}}=O\left(N_{n}^{-1 / 2}\right)$ in view of the standard Berry-Esseen inequality. Moreover, since $\operatorname{tr}\left(\Gamma_{n, k}^{4}\right) \leq\left\|\Gamma_{n, k}\right\|_{\mathrm{sp}}^{2}\left\|\Gamma_{n, k}\right\|_{F}^{2}$, we might expect $\max _{1 \leq k \leq d_{n}} \operatorname{tr}\left(\Gamma_{n, k}^{4}\right)=$ $O\left(N_{n}^{-1}\right)$ due to a similar reason. Consequently, the condition (3.3) is typically satisfied when $\log ^{10} d_{n}=o\left(N_{n}\right)$ as $n \rightarrow \infty$.

A typical example satisfying the above condition is the situations where $\Gamma_{n, k}$ 's correspond to sample autocovariances:

$$
\gamma_{n, k}(i, j)= \begin{cases}1 / \sqrt{N_{n}} & \text { if }|j-i|=k \\ 0 & \text { otherwise }\end{cases}
$$

In this case the quantities $\sum_{j=1}^{N_{n}} \gamma_{n, k}(i, j)^{2}$ does not depend on $k$, so we can take $\bar{\Gamma}_{n}=\Gamma_{n, 1}$ for example.

\section{Application to high-frequency data}

\subsection{Testing the absence of lead-lag effects}

We turn to the problem of testing the absence of lead-lag effects which is mentioned at the beginning of the Introduction. Here we consider a more general setting than the one described in the Introduction by allowing (deterministic) time-varying volatilities as well as the presence of multiple lead-lag times under the alternative.

Let $\rho_{1}, \ldots, \rho_{M}$ be real numbers satisfying the condition $\sum_{m=1}^{M}\left|\rho_{m}\right|<1$. Also, let $\theta_{1}, \ldots, \theta_{M}$ be mutually different numbers. Then, by Proposition 2 from [26] there is a bivariate Gaussian process $B_{t}=\left(B_{t}^{1}, B_{t}^{2}\right)(t \in \mathbb{R})$ with stationary increments such that both $B^{1}$ and $B^{2}$ are standard Brownian motions as well as $B^{1}$ and $B^{2}$ have the cross spectral density given by

$$
\mathfrak{s}(\lambda)=\sum_{m=1}^{M} \rho_{m} e^{-\sqrt{-1} \theta_{m} \lambda}, \quad \lambda \in \mathbb{R} .
$$


This means that we have

$$
E\left[\left(\int_{-\infty}^{\infty} f(t) d B_{t}^{1}\right)\left(\int_{-\infty}^{\infty} g(t) d B_{t}^{2}\right)\right]=\sum_{m=1}^{M} \rho_{m} \int_{-\infty}^{\infty} f(t) g\left(t+\theta_{m}\right) d t
$$

for any $f, g \in L^{2}(\mathbb{R})$.

For each $\nu=1,2$, we consider the process $X^{\nu}=\left(X_{t}^{\nu}\right)_{t \geq 0}$ given by

$$
X_{t}^{\nu}=X_{0}^{\nu}+\int_{0}^{t} \sigma_{\nu}(s) d B_{s}^{\nu}, \quad t \geq 0
$$

where $\sigma_{\nu} \in L^{2}(0, \infty)$ is nonnegative-valued and deterministic. We observe the process $X^{\nu}$ on the interval $[0, T]$ at the deterministic sampling times $0 \leq t_{0}^{\nu}<t_{1}^{\nu}<\cdots<t_{n_{\nu}}^{\nu} \leq T$, which implicitly depend on the parameter $n \in \mathbb{N}$ such that

$$
r_{n}:=\max _{\nu=1,2} \max _{i=0,1, \ldots, n_{\nu}+1}\left(t_{i}^{\nu}-t_{i-1}^{\nu}\right) \rightarrow 0
$$

as $n \rightarrow \infty$, where we set $t_{-1}^{\nu}:=0$ and $t_{n_{\nu}+1}^{\nu}:=T$ for each $\nu=1,2$.

Remark 4.1. It is not difficult to extend the following discussion to the case that the volatilities $\sigma_{1}, \sigma_{2}$ and the sampling times $\left(t_{i}^{1}\right)_{i=0}^{n_{1}},\left(t_{j}^{2}\right)_{j=0}^{n_{2}}$ are random but independent of the process $B$, but we focus on the deterministic case for the simplicity of notation. Extension to a situation where the volatilities depend on $B$ is non-trivial because of the non-ergodic nature of the problem (i.e. the asymptotic covariance matrix of the statistics $\left(U_{n}(\theta)\right)_{\theta \in \mathcal{G}_{n}}$ defined below generally depends on $B$ ) and we leave it to future research.

Our aim is to construct a testing procedure for the following statistical hypothesis testing problem based on discrete observation data $\left(X_{t_{i}^{1}}^{1}\right)_{i=0}^{n_{1}}$ and $\left(X_{t_{i}^{2}}^{2}\right)_{i=0}^{n_{2}}$ :

$$
H_{0}: \rho_{m}=0 \text { for all } m=1, \ldots, M \quad \text { vs } \quad H_{1}: \rho_{m} \neq 0 \text { for some } m=1, \ldots, M \text {. }
$$

We introduce some notation. For each $\nu=1,2$, we associate the observation times $\left(t_{i}^{\nu}\right)_{i=0}^{n_{\nu}}$ with the collection of intervals $\Pi_{n}^{\nu}=\left\{\left(t_{i-1}^{\nu}, t_{i}^{\nu}\right]: i=1, \ldots, n_{\nu}\right\}$. We will systematically employ the notation $I$ (resp. $J$ ) for an element of $\Pi_{n}^{1}\left(\right.$ resp. $\left.\Pi_{n}^{2}\right)$. For an interval $S \subset[0, \infty)$, we set $\bar{S}=\sup S, \underline{S}=\inf S$ and $|S|=\bar{S}-\underline{S}$. In addition, we set $V(S)=V_{\bar{S}}-V_{\underline{S}}$ for a a stochastic process $\left(V_{t}\right)_{t \geq 0}$, and $S_{\theta}=S+\theta$ for a real number $\theta$. We define the Hoffmann-Rosenbaum-Yoshida cross-covariance estimator by

$$
U_{n}(\theta)=\sum_{I \in \Pi_{n}^{1}, J \in \Pi_{n}^{2}} X^{1}(I) X^{2}(J) K\left(I, J_{-\theta}\right)
$$

where we set $K(I, J)=1_{\{I \cap J \neq \emptyset\}}$ for two intervals $I$ and $J$. Now our test statistic is given by

$$
T_{n}=\sqrt{n} \max _{\theta \in \mathcal{G}_{n}}\left|U_{n}(\theta)\right|,
$$

where $\mathcal{G}_{n}$ is a finite subset of $\mathbb{R}$.

To establish the asymptotic property of our test statistic $T_{n}$, we first investigate the asymptotic property of the following quantity:

$$
F_{n}(\theta)=\sqrt{n}\left(U_{n}(\theta)-E\left[U_{n}(\theta)\right]\right) .
$$

We impose the following conditions: 
[A1] $\sup _{t \in[0, T]}\left(\sigma_{1}(t)+\sigma_{2}(t)\right)<\infty$.

[A2] There are positive constants $\underline{v}, \bar{v}$ such that $\underline{v} \leq V_{n}(\theta) \leq \bar{v}$ for all $n \in \mathbb{N}$ and $\theta \in \mathcal{G}_{n}$, where

$$
V_{n}(\theta)=n \sum_{I \in \Pi_{n}^{1}, J \in \Pi_{n}^{2}}\left(\int_{I} \sigma_{1}(t)^{2} d t\right)\left(\int_{J} \sigma_{2}(t)^{2} d t\right) K\left(I, J_{-\theta}\right) .
$$

[A3] $\Sigma\left(\theta_{m}\right)>0$ for all $m=1, \ldots, M$, where

$$
\Sigma(\theta)= \begin{cases}\int_{0}^{T-\theta} \sigma_{1}(t) \sigma_{2}(t+\theta) d t & \text { if } \theta \geq 0 \\ \int_{0}^{T+\theta} \sigma_{1}(t-\theta) \sigma_{2}(t) d t & \text { if } \theta<0 .\end{cases}
$$

[A4] The grid $\mathcal{G}_{n}$ satisfies the following conditions:

(i) There is a constant $\gamma>0$ such that $\# \mathcal{G}_{n}=O\left(n^{\gamma}\right)$ as $n \rightarrow \infty$.

(ii) There is a sequence $\left(v_{n}\right)_{n \in \mathbb{N}}$ of positive numbers such that

$$
\left\{\theta_{1}, \ldots, \theta_{M}\right\} \subset \bigcup_{\theta \in \mathcal{G}_{n}}\left[\theta-v_{n}, \theta+v_{n}\right]
$$

and $\lim _{n \rightarrow \infty} v_{n} \min \left\{n_{1}, n_{2}\right\}=0$.

Remark 4.2. Assumption [A1] is standard in the literature and satisfied when $\sigma_{1}$ and $\sigma_{2}$ are càdlàg, for example. [A2] roughly says that the scaling factor $\sqrt{n}$ is appropriate (the quantity $V_{n}(\theta)$ is related to the variance of $U_{n}(\theta)$ ). [A2] holds true e.g. when $0<\inf _{t \in[0, T]} \sigma_{\nu}(t) \leq \sup _{t \in[0, T]} \sigma_{\nu}(t)<\infty$ for every $\nu=1,2, n \sum_{I \in \Pi_{n}^{1}}|I|^{2}+$ $n \sum_{J \in \Pi_{n}^{2}}|J|^{2}=O(1)$ as $n \rightarrow \infty$ and there is a constant $c>0$ such that $n(|I| \wedge|J|) \geq c$ for every $n$ and all $I \in \Pi_{n}^{1}, J \in \Pi_{n}^{2}$. [A3] ensures that $\max _{1 \leq m \leq M}\left|E\left[U_{n}\left(\theta_{m}\right)\right]\right|$ does not vanish under $H_{1}$. [A4] ensures that $\mathcal{G}_{n}$ is sufficiently fine to capture the cross-covariance at the lag $\theta_{m}$ for every $m$. Note that [A4] is also assumed in [30] (see Assumption B3 of [30]).

Proposition 4.1. For each $n \in \mathbb{N}$, let $\left(Z_{n}(\theta)\right)_{\theta \in \mathcal{G}_{n}}$ be a family of centered Gaussian variables such that $E\left[Z_{n}(\theta) Z_{n}\left(\theta^{\prime}\right)\right]=$ $E\left[F_{n}(\theta) F_{n}\left(\theta^{\prime}\right)\right]$ for all $\theta, \theta^{\prime} \in \mathcal{G}_{n}$. Under assumptions [A1]-[A2], we have

$$
\sup _{x \in \mathbb{R}}\left|P\left(\max _{\theta \in \mathcal{G}_{n}}\left|F_{n}(\theta)\right| \leq x\right)-P\left(\max _{\theta \in \mathcal{G}_{n}}\left|Z_{n}(\theta)\right| \leq x\right)\right| \rightarrow 0
$$

as $n \rightarrow \infty$, provided that $n r_{n}^{2} \log ^{6}\left(\# \mathcal{G}_{n}\right) \rightarrow 0$.

Remark 4.3. It is impossible to apply the original CCK theory (at least naively) to prove Proposition 4.1 because we need to apply Theorem 3.1 to a situation where the matrices $\Sigma_{n}^{1 / 2} A_{n, 1} \Sigma_{n}^{1 / 2}, \ldots, \Sigma_{n}^{1 / 2} A_{n, d_{n}} \Sigma_{n}^{1 / 2}$ are not simultaneously diagonalizable. In fact, if we consider the synchronous and equidistant sampling with the step size $1 / n$, the matrices corresponding to $U_{n}( \pm 1 / n)$ are of the form

$$
\left(\begin{array}{cc}
O & A \\
A^{\top} & O
\end{array}\right)
$$

where we take the matrix $A=\left(a_{i j}\right)$ as

$$
a_{i j}= \begin{cases}1 & \text { if } j-i= \pm 1 \\ 0 & \text { otherwise }\end{cases}
$$

We can easily check that those matrices are not commutative unless the size of $A$ is less than or equal to 2. 
The above proposition suggests that the null distribution of our test statistic $T_{n}$ could be approximated by that of $\max _{\theta \in \mathcal{G}_{n}}\left|Z_{n}(\theta)\right|$ for sufficiently large $n$. However, it is not easy to evaluate the distribution of $\max _{\theta \in \mathcal{G}_{n}}\left|Z_{n}(\theta)\right|$ directly, so we rely on a (wild) bootstrap procedure to construct critical regions for our test. The above Gaussian approximation result plays a role in validating the bootstrap procedure.

Let $\left(w_{I}^{1}\right)_{I \in \Pi_{n}^{1}}$ and $\left(w_{J}^{2}\right)_{J \in \Pi_{n}^{2}}$ be mutually independent sequence of i.i.d. random variables which are independent of the processes $X^{1}$ and $X^{2}$. Then we set

$$
U_{n}^{*}(\theta)=\sum_{I \in \Pi_{n}^{1}, J \in \Pi_{n}^{2}}\left(w_{I}^{1} X^{1}(I)\right)\left(w_{J}^{2} X^{2}(J)\right) K\left(I, J_{-\theta}\right) .
$$

Given a significance level $\alpha$, we denote by $q_{n}^{*}(1-\alpha)$ the $100(1-\alpha) \%$ quantile of the bootstrapped test statistic $T_{n}^{*}=\sqrt{n} \max _{\theta \in \mathcal{G}_{n}}\left|U_{n}^{*}(\theta)\right|$, conditionally on $X^{1}$ and $X^{2}$ :

$$
q_{n}^{*}(1-\alpha)=\inf \left\{z \in \mathbb{R}: P\left(T_{n}^{*} \leq z \mid \mathcal{F}^{X}\right) \geq 1-\alpha\right\},
$$

where $\mathcal{F}^{X}$ is the $\sigma$-field generated by the processes $X^{1}$ and $X^{2}$.

Remark 4.4. We generate the bootstrap observations under the null hypothesis $H_{0}$. This is a typical approach in the bootstrap test literature (see e.g. [5]). Moreover, as discussed in Section 4 of [21] as well as Section 2 of [20], this approach often serves as refining the performance of the test.

Proposition 4.2. Suppose that $[\mathrm{A} 1]-[\mathrm{A} 4]$ are satisfied. Suppose also that $E\left[w_{I}^{1}\right]=E\left[w_{J}^{2}\right]=0, E\left[\left(w_{I}^{1}\right)^{2}\right]=$ $E\left[\left(w_{J}^{2}\right)^{2}\right]=1$ for all $I, J$ and there is a constant $a>0$ such that both $w_{I}^{1}$ and $w_{J}^{2}$ are sub-Gaussian relative to the scale a for all I, J. Suppose further that $r_{n}=O\left(n^{-3 / 4-\eta}\right)$ as $n \rightarrow \infty$ for some $\eta>0$. Then the following statements hold true for all $\alpha \in(0,1)$ :

(a) Under $H_{0}$, we have $P\left(T_{n} \geq q_{n}^{*}(1-\alpha)\right) \rightarrow \alpha$ as $n \rightarrow \infty$.

(b) Under $H_{1}$, we have $P\left(T_{n} \geq q_{n}^{*}(1-\alpha)\right) \rightarrow 1$ as $n \rightarrow \infty$.

By Proposition 4.2, given a significance level $\alpha \in(0,1)$, we obtain a consistent and asymptotically level $\alpha$ test for (4.2) by rejecting the null hypothesis if $T_{n} \geq q_{n}^{*}(1-\alpha)$. Of course, in the practical implementation we replace $q_{n}^{*}(1-\alpha)$ by a simulated one. For example, given observation data, we generate i.i.d. copies $T_{n}^{*}(1), \ldots, T_{n}^{*}(R)$ of $T_{n}^{*}$ (conditionally on the observation data) with some sufficiently large integer $R$. Then we replace the function $P\left(T_{n}^{*} \leq z \mid \mathcal{F}^{X}\right)$ of $z$ by its empirical counterpart $\frac{1}{R} \sum_{r=1}^{R} 1_{\left\{T_{n}^{*}(r) \leq z\right\}}$ and compute $q_{n}^{*}(1-\alpha)$ accordingly. Note that this is equivalent to computing the bootstrap p-value $\hat{p}^{*}=\frac{1}{R} \sum_{r=1}^{R} 1_{\left\{T_{n}^{*}(r)>T_{n}\right\}}$ and rejecting the null hypothesis if $\hat{p}^{*} \leq \alpha$.

Remark 4.5. The proposed test is evidently invariant under multiplying a constant. In particular, the factor $\sqrt{n}$ can be dropped when we implement the test in practice.

Remark 4.6 (Choice of the multiplier variables). Choice of the distribution of the multiplier variables $\left(w_{I}^{1}\right)_{I \in \Pi_{n}^{1}}$ and $\left(w_{J}^{2}\right)_{J \in \Pi_{n}^{2}}$ are important for the finite sample property of the test. In our situation it turns out that choosing Rademacher variables induces a quite good finite sample performance of our testing procedure. Namely, the proposed test performs very well in finite samples when the distributions of $w_{I}^{1}$ and $w_{J}^{2}$ are chosen according to

$$
P\left(w_{I}^{1}=1\right)=P\left(w_{I}^{1}=-1\right)=P\left(w_{J}^{2}=1\right)=P\left(w_{J}^{2}=-1\right)=\frac{1}{2} .
$$


This is presumably because the above choice makes the unconditional distribution of the bootstrapped test statistics of $T_{n}^{*}$ coincide with the distribution of $T_{n}$. This can be shown in the same way as in the proof of Theorem 1 from [20]. For this reason we recommend that we should use Rademacher variables as the multiplier variables for the above testing procedure (and we do so in the simulation study of Section 5).

\subsection{Uniform confidence bands for spot volatility}

To illustrate another potential application of our main results, we present an application of our result to constructing uniform confidence bands for spot volatility. This section is only for an illustration purpose, so we do neither pursue the generality of the theory nor discuss practical problems on implementation such as the choice of a bandwidth and a kernel function. We refer to Section 6 of [38] for a discussion on the latter issue.

Let us consider the stochastic process $X=\left(X_{t}\right)_{t \in[0, T]}$ which is defined on a stochastic basis $\left(\Omega, \mathcal{F},\left(\mathcal{F}_{t}\right)_{t \in[0, T]}, P\right)$ and of the form

$$
X_{t}=X_{0}+\int_{0}^{t} \sigma(s) d B_{s}, \quad t \in[0, T]
$$

where $B=\left(B_{t}\right)_{t \in[0, T]}$ is a standard $\left(\mathcal{F}_{t}\right)$-Brownian motion and $\sigma=(\sigma(t))_{t \in[0, T]}$ is a continuous $\left(\mathcal{F}_{t}\right)$-adapted process. The aim of this section is to construct uniform confidence bands for the spot volatility $\sigma^{2}$ based on the high-frequency observation data $\left\{X_{t_{i}}\right\}_{i=0}^{n}$, where $t_{i}=T i / n, i=0,1, \ldots, n$.

Specifically, we consider the following kernel-type estimator for $\sigma^{2}$ (cf. [24, 38]):

$$
\widehat{\sigma}_{n}^{2}(t):=\sum_{i=1}^{n} K_{h}\left(t_{i-1}-t\right)\left(X_{t_{i}}-X_{t_{i-1}}\right)^{2}, \quad t \in[0, T],
$$

where $h:=h_{n}>0$ is a bandwidth parameter, $K_{h}(x)=K(x / h) / h$ for $x \in \mathbb{R}$ and $K: \mathbb{R} \rightarrow \mathbb{R}$ is a kernel function. We derive a Gaussian approximation result for the supremum of the Studentization of $\widehat{\sigma}_{n}^{2}(t)$. Let us set

$$
\mathfrak{s}_{n}(t)=\sqrt{\frac{2}{n^{2}} \sum_{i=1}^{n} K_{h}\left(t_{i-1}-t\right)^{2}}
$$

for $t \in[0, T]$. In view of Theorem 3 from [38], $\sigma^{2}(t) \mathfrak{s}_{n}(t)$ can be seen as an approximation of the asymptotic standard error of $\widehat{\sigma}_{n}^{2}(t)$. We define the Gaussian analog of the Studentization of $\widehat{\sigma}_{n}^{2}(t)$ as follows. For each $n \in \mathbb{N}$, let $\left(z_{i}^{n}\right)_{i=1}^{n}$ be a sequence of i.i.d. centered Gaussian variables with variance $2 / n^{2}$, and we set

$$
Z_{n}(t)=\frac{1}{\mathfrak{s}_{n}(t)} \sum_{i=1}^{n} K_{h}\left(t_{i-1}-t\right) z_{i}^{n}, \quad t \in[0, T] .
$$

We impose the following conditions:

[B1] $w(\sigma ; \eta)=O_{p}\left(\eta^{\gamma}\right)$ as $\eta \rightarrow 0$ for some $\gamma \in(0,1]$. Moreover, $\sigma^{2}(t)>0$ for all $t \in[0, T]$ almost surely.

[B2] The kernel function $K: \mathbb{R} \rightarrow \mathbb{R}$ is Lipschitz continuous and compactly supported as well as satisfies $\int_{-\infty}^{\infty} K(t) d t=1$.

We also impose the following strengthened version of assumption [B1] when deriving the convergence rate of Gaussian approximation:

[SB1] There is a constant $\Lambda>0$ such that $\Lambda^{-1} \leq|\sigma(t)| \leq \Lambda$ and $w(\sigma ; \eta) \leq \Lambda \eta^{\gamma}$ for all $t \in[0, T]$ and $\eta \in(0,1)$. 
Proposition 4.3. Suppose that [B1]-[B2] are satisfied. Suppose also that the bandwidth parameter $h$ satisfies $n h^{1+2 \gamma} \log n \rightarrow 0$ and $\log ^{6} n / n h \rightarrow 0$ as $n \rightarrow \infty$. Let $a_{n}$ be a sequence of positive numbers such that $a_{n} \rightarrow 0$ and $a_{n} / h \rightarrow \infty$ as $n \rightarrow \infty$. Then we have

$$
\sup _{x \in \mathbb{R}}\left|P\left(\sup _{t \in\left[a_{n}, T-a_{n}\right]}\left|\frac{\widehat{\sigma}_{n}^{2}(t)-\sigma^{2}(t)}{\sigma^{2}(t) \mathfrak{s}_{n}(t)}\right| \leq x\right)-P\left(\sup _{t \in\left[a_{n}, T-a_{n}\right]}\left|Z_{n}(t)\right| \leq x\right)\right| \rightarrow 0
$$

as $n \rightarrow \infty$. Moreover, if we additionally assume [SB1], we have

$$
\begin{array}{r}
\sup _{x \in \mathbb{R}}\left|P\left(\sup _{t \in\left[a_{n}, T-a_{n}\right]}\left|\frac{\widehat{\sigma}_{n}^{2}(t)-\sigma^{2}(t)}{\sigma^{2}(t) \mathfrak{s}_{n}(t)}\right| \leq x\right)-P\left(\sup _{t \in\left[a_{n}, T-a_{n}\right]}\left|Z_{n}(t)\right| \leq x\right)\right| \\
=O\left(\sqrt{n h^{1+2 \gamma} \log n}\right)+O\left(\frac{\log n}{(n h)^{\frac{1}{6}}}\right)
\end{array}
$$

as $n \rightarrow \infty$.

Remark 4.7. We introduce the parameters $a_{n}$ in Proposition 4.3 to avoid boundary effects. See Section 4 of [38] for more details about this topic.

Remark 4.8. Although we use Lemma 2.1 to prove Proposition 4.3 (see the proof of Lemma B.17), we can indeed use Theorem 3.1 of [16] instead to derive a similar result. However, the result requires a (slightly) stronger condition on the bandwidth $h$ and leads to a worse convergence rate. In fact, an inspection of the proof of Proposition 2.1 from [17] implies that we need to replace $\varepsilon^{-2}$ by $\varepsilon^{-3}$ in the inequality (B.9) if we use Theorem 3.1 of [16] instead of Lemma 2.1 to prove Lemma B.17. Then, the optimal choice of $\varepsilon$ in the proof of Proposition 4.3 becomes $\varepsilon=(n h)^{-\frac{1}{8}} \log ^{3 / 8} n$, which changes the order of the second term on the right side of (4.4) to $O\left((n h)^{-\frac{1}{8}} \log ^{7 / 8} n\right)$. Hence we need the condition $\log ^{7} n / n h \rightarrow 0$ as $n \rightarrow \infty$ to get the convergence (4.3).

In contrast to the previous subsection, the process $Z_{n}(t)$ does not contain any unknown parameter, so Proposition 4.3 is readily applicable to construction of uniform confidence bands for $\sigma^{2}$ : Given a significance level $\alpha \in(0,1)$, let $q_{n}(1-\alpha)$ be the $100(1-\alpha) \%$ quantile of the variable $\sup _{t \in\left[a_{n}, T-a_{n}\right]}\left|Z_{n}(t)\right|$ (which can be computed e.g. by simulation). Then,

$$
\left[\frac{\widehat{\sigma}_{n}^{2}(t)}{1+\mathfrak{s}_{n}(t) \cdot q_{n}(1-\alpha)}, \frac{\widehat{\sigma}_{n}^{2}(t)}{1-\mathfrak{s}_{n}(t) \cdot q_{n}(1-\alpha)}\right], \quad t \in\left[a_{n}, T-a_{n}\right]
$$

give asymptotically uniformly valid $100(1-\alpha) \%$ confidence bands for $\sigma^{2}(t), t \in\left[a_{n}, T-a_{n}\right]$.

Remark 4.9. The applications considered in this section concerns asymptotic settings where the terminal value $T$ of the sampling interval is fixed. Here, we briefly discuss applicability of our theory to asymptotic settings where the terminal value $T$ of the sampling interval tends to infinity. In such a setting, a typical problem which our theory seems to fit would be constructing uniform confidence bands for the coefficient functions of an ergodic diffusion process. Non-parametric estimation of the coefficient functions of a diffusion process from high-frequency data is extensively studied in the literature, but most studies focus only on point-wise inference (except for Kanaya [33], where uniform convergence rates of kernel-based estimators have been derived; see also Söhl and Trabs [58] where the authors construct uniform confidence bands for the drift coefficient of a diffusion process based on lowfrequency observation data), so it would be important to consider this problem. In such a problem, estimators 
typically have deterministic asymptotic covariance matrices, hence the issue indicated in Remark 4.1 does not arise. Unfortunately, however, we encounter another issue that it seems difficult (at least not straightforward) to get a reasonable estimate for the quantity $\Delta$ in this problem. This is perhaps because we do not take account of special properties of the underlying diffusion process (such as the Markov and mixing properties) when deriving our estimate. Therefore, this issue might be resolved by adopting the approach from Kusuoka and Yoshida [39] where Malliavin calculus is locally applied to the underlying process with taking account of the Markov and mixing properties. However, a rigorous treatment of this idea is rather demanding, so we leave it to future work.

\section{Numerical illustration}

In this section we illustrate the finite sample performance of the testing procedure for the absence of lead-lag effects, which is proposed in Section 4.1.2 The setting of our numerical experiments is basically adopted from Section 5 of [30]. Specifically, we simulate model (1.1) with $T=1, \vartheta=0.1, x_{0}^{1}=x_{0}^{2}=0, \sigma_{1}=\sigma_{2}=1$. We vary the correlation parameter as $\rho \in\{0,0.25,0.5,0.75\}$ to examine the size and the power of the testing procedure. We consider both synchronous and non-synchronous sampling scenarios. For the synchronous sampling scenario $t_{i}^{1}=$ $t_{i}^{2}=i h_{n}, i=0,1, \ldots,\left\lfloor T h_{n}^{-1}\right\rfloor$, we examine three kinds of sampling frequencies: $h_{n} \in\left\{10^{-3}, 3 \cdot 10^{-3}, 6 \cdot 10^{-3}\right\}$. Also, in these scenarios we set $\mathcal{G}_{n}=\left\{k h_{n}: k \in \mathbb{Z},\left|k h_{n}\right| \leq 0.3\right\}$ as the search grid. On the other hand, for the nonsynchronous sampling scenario, we first simulate the processes on the equidistant times $i \cdot 10^{-3}, i=0,1, \ldots, 1,000$, then we randomly pick 300 sampling times for $X^{1}$. We do so for $X^{2}$ independently of the sampling for $X^{1}$. In this scenario we set $\mathcal{G}_{n}=\left\{k \cdot 10^{-3}: k \in \mathbb{Z},|k| \leq 300\right\}$ as the search grid. For the testing procedure, we use Rademacher variables as the multiplier variables and 999 bootstrap replications to construct the critical regions. We run 10,000 Monte Carlo iterations in each experiment.

Table 1 reports the rejection rates of the proposed test in each experiment. For the case $\rho=0$, the numbers should be close to the corresponding significance levels, and this is true for all the experiments. Turning to the power performance, we find that in the low correlation case $\rho=0.25$ the power of the test is rather weak except for the most frequent sampling scenario. This is reasonable in view of the simulation results reported in [30], which indicate that the contrast function $U_{n}(\theta)$ becomes flat in that case. For the moderate and the high correlation cases $\rho=0.5$ and $\rho=0.75$, the power of the test is satisfactory.

\footnotetext{
${ }^{2}$ The proposed testing procedure is implemented in the $\mathrm{R}$ package yuima as the function llag. test since version 1.7.2.
} 
Table 1: Rejection rates of the proposed test

\begin{tabular}{|c|c|c|c|c|}
\hline & $\rho=0$ & $\rho=0.25$ & $\rho=0.50$ & $\rho=0.75$ \\
\hline \multicolumn{5}{|c|}{ Synchronous sampling scenario } \\
\hline \multicolumn{5}{|l|}{$h_{n}=10^{-3}$} \\
\hline$\alpha=0.01$ & 0.011 & 1.000 & 1.000 & 1.000 \\
\hline$\alpha=0.05$ & 0.050 & 1.000 & 1.000 & 1.000 \\
\hline$\alpha=0.10$ & 0.100 & 1.000 & 1.000 & 1.000 \\
\hline \multicolumn{5}{|c|}{$h_{n}=3 \cdot 10^{-3}$} \\
\hline$\alpha=0.01$ & 0.010 & 0.139 & 0.977 & 1.000 \\
\hline$\alpha=0.05$ & 0.051 & 0.281 & 0.993 & 1.000 \\
\hline$\alpha=0.10$ & 0.101 & 0.382 & 0.997 & 1.000 \\
\hline \multicolumn{5}{|c|}{$h_{n}=6 \cdot 10^{-3}$} \\
\hline$\alpha=0.01$ & 0.011 & 0.041 & 0.634 & 0.997 \\
\hline$\alpha=0.05$ & 0.050 & 0.131 & 0.802 & 1.000 \\
\hline$\alpha=0.10$ & 0.099 & 0.214 & 0.867 & 1.000 \\
\hline \multicolumn{5}{|c|}{ Non-synchronous sampling scenario } \\
\hline$\alpha=0.01$ & 0.010 & 0.056 & 0.753 & 1.000 \\
\hline$\alpha=0.05$ & 0.051 & 0.152 & 0.879 & 1.000 \\
\hline$\alpha=0.10$ & 0.099 & 0.235 & 0.919 & 1.000 \\
\hline
\end{tabular}

Note. $\alpha$ denotes the significance level.

\section{Appendix}

\section{A Preliminaries}

\section{A.1 Basic elements of Gaussian analysis and Malliavin calculus}

In this section we briefly overview the basic elements of Gaussian analysis and Malliavin calculus for Gaussian processes. See [32, 48, 52] for more details about these topics.

Throughout the paper, $H$ denotes a real separable Hilbert space. The inner product and the norm of $H$ are denoted by $\langle\cdot, \cdot\rangle_{H}$ and $\|\cdot\|_{H}$, respectively. We assume that an isonormal Gaussian process $W=(W(h))_{h \in H}$ over $H$ defined on a probability space $(\Omega, \mathcal{F}, P)$ is given. Namely, $W$ is a centered Gaussian family of random variables such that $E[W(h) W(g)]=\langle h, g\rangle_{H}$ for any $h, g \in H$.

We denote by $L^{2}(W)$ the space $L^{2}(\Omega, \sigma(W), P)$ for short. For every non-negative integer $q$, we denote by $\mathcal{H}_{q}$ the closed subspace of $L^{2}(W)$ spanned by the set $\left\{\operatorname{He}_{q}(W(h)): h \in H,\|h\|_{H}=1\right\}$, where $\operatorname{He}_{q}(x)=$ $(-1)^{q} e^{x^{2} / 2} \frac{d^{q}}{d x^{q}}\left(e^{-x^{2} / 2}\right)$ is the $q$ th Hermite polynomial. The space $\mathcal{H}_{q}$ is called the $q$ th Wiener chaos of $W$. It is well-known that the spaces $\mathcal{H}_{q}$ and $\mathcal{H}_{r}$ are orthogonal whenever $q \neq r$ (cf. Lemma 1.1.1 of [52]). Moreover, the space $L^{2}(W)$ possesses the following orthogonal decomposition: $L^{2}(W)=\oplus_{q=0}^{\infty} \mathcal{H}_{q}$ (cf. Theorem 1.1.1 of [52]). This decomposition is called the Wiener-Itô chaos decomposition of $L^{2}(W)$. We denote by $J_{q}$ the orthogonal projection of $L^{2}(W)$ onto $\mathcal{H}_{q}$ for each $q$. Therefore, every $F \in L^{2}(W)$ has the decomposition $F=\sum_{q=0}^{\infty} J_{q} F$, which is called the Wiener-Itô chaos decomposition of $F$. 
A closed subspace $\mathbb{H}$ of $L^{2}(\Omega, \mathcal{F}, P)$ is called a Gaussian Hilbert space if all the elements of $\mathbb{H}$ are centered Gaussian variables. We can easily check that $\{W(h): h \in H\}$ is a Gaussian Hilbert space. Given a Gaussian Hilbert space $\mathbb{H}$ and an integer $q \geq 0$, we set

$$
\mathcal{P}_{q}(\mathbb{H})=\left\{\varphi\left(\xi_{1}, \ldots, \xi_{m}\right): \varphi \text { is a polynomial of degree } \leq q ; \xi_{1}, \ldots, \xi_{m} \in \mathbb{H} ; m \in \mathbb{N}\right\}
$$

and denote by $\overline{\mathcal{P}}_{q}(\mathbb{H})$ the closure of $\mathcal{P}_{q}(\mathbb{H})$ in $L^{2}(\Omega, \mathcal{F}, P)$. If $\mathbb{H}=\{W(h): h \in H\}, \overline{\mathcal{P}}_{q}(\mathbb{H})$ coincides with the space $\oplus_{p=0}^{q} \mathcal{H}_{p}$ (cf. page 6 of [52]). The importance of the Gaussian Hilbert space in this paper is illuminated by Proposition A.1.

For a non-negative integer $q, H^{\otimes q}$ and $H^{\odot q}$ denote the $q$ th tensor power and $q$ th symmetric tensor power, respectively (see Appendix E of [32] for details). The qth multiple Wiener-Itô integral $I_{q}: H^{\odot q} \rightarrow \mathcal{H}_{q}$ is defined as the unique linear isometry between $H^{\odot q}$ (equipped with the scaled norm $\sqrt{q !}\|\cdot\|_{H^{\otimes q}}$ ) and $\mathcal{H}_{q}$ (equipped with the norm of $L^{2}(W)$ ) such that $I_{q}\left(h^{\otimes q}\right)=\mathrm{He}_{q}(W(h))$ for all $h \in H$ with $\|h\|_{H}=1$.

Let $p, q$ be positive integers. For $f \in H^{\odot p}, g \in H^{\odot q}$ and $r \in\{0,1, \ldots, p \wedge q\}, f \otimes_{r} g$ denotes the $r$ th contraction of $f$ and $g$. The symmetrization of $f \otimes_{r} g$ is denoted by $f \widetilde{\otimes}_{r} g$. See Appendix B of [48] for more details on these concepts.

A random variable $F$ is said to be smooth if it can be written as

$$
F=f\left(W\left(h_{1}\right), \ldots, W\left(h_{m}\right)\right)
$$

where $h_{1}, \ldots, h_{m} \in H$ and $f: \mathbb{R}^{m} \rightarrow \mathbb{R}$ is a $C^{\infty}$ function such that $f$ and all of its partial derivatives have at most polynomial growth. We denote by $\mathcal{S}$ the set of all smooth random variables. For a smooth random variable $F$ of the form (A.1) and an integer $k \geq 1$, we define the $k$ th Malliavin derivative of $F$ as the $H^{\odot k}$-valued random variable defined by

$$
D^{k} F=\sum_{i_{1}, \ldots, i_{k}=1}^{m} \frac{\partial^{k} f}{\partial x_{i_{1}} \ldots \partial x_{i_{k}}}\left(W\left(h_{1}\right), \ldots, W\left(h_{m}\right)\right) h_{i} .
$$

For a real number $p \geq 1$, let us denote by $L^{p}\left(\Omega ; H^{\odot k}\right)$ the set of all $H^{\odot k}$-valued random variables $Y$ such that $E\left[\|Y\|_{H^{\otimes k}}^{p}\right]<\infty$. We regard $L^{p}\left(\Omega ; H^{\odot k}\right)$ as the Banach space equipped with the norm defined by $\|Y\|_{L^{p}\left(\Omega ; H^{\odot k}\right)}=$ $\left(E\left[\|Y\|_{H \otimes k}^{p}\right]\right)^{1 / p}$. Then, it is well-known that the $k$ th Malliavin derivative operator $D^{k}$ on $\mathcal{S} \subset L^{p}(\Omega, \mathcal{F}, P)$ into $L^{p}\left(\Omega ; H^{\odot k}\right)$ is closable (cf. Proposition 2.3.4 of [48]). Therefore, there is a unique closed operator on $\mathbb{D}_{k, p} \subset L^{p}(\Omega, \mathcal{F}, P)$ into $L^{p}\left(\Omega ; H^{\odot k}\right)$, which is also denoted by $D^{k}$, such that its graph is equal to the closure of $\mathcal{S}$ with respect to the norm

$$
\|F\|_{k, p}=\left(E\left[|F|^{p}\right]+\sum_{j=1}^{k} E\left[\left\|D^{j} F\right\|_{H^{\otimes j}}^{p}\right]\right)^{1 / p} .
$$

We write $D$ instead of $D^{1}$ for short. Malliavin derivatives enjoy the following chain rule (cf. Proposition 1.2.3 of [52]): Let $F_{1}, \ldots, F_{m} \in \mathbb{D}_{1, p}$ and let $\varphi: \mathbb{R}^{m} \rightarrow \mathbb{R}$ be a $C^{1}$ function with bounded partial derivatives. Then $\varphi\left(F_{1}, \ldots, F_{m}\right) \in \mathbb{D}_{1, p}$ and we have

$$
D \varphi\left(F_{1}, \ldots, F_{m}\right)=\sum_{i=1}^{m} \frac{\partial \varphi}{\partial x_{i}}\left(F_{1}, \ldots, F_{m}\right) D F_{i} .
$$


We also note that, for any integer $q \geq 1$ and $f \in H^{\odot q}, I_{q}(f) \in \mathbb{D}_{1, p}$ and $D I_{q}(f)=q I_{q-1}(f)$ (cf. Proposition 2.7.4 of [48]).

We denote by $\delta$ the divergence operator, which is the adjoint of the operator $D$ on $\mathbb{D}_{1,2} \subset L^{2}(W)$ into $L^{2}(\Omega ; H)$. The domain of $\delta$ is denoted by $\operatorname{Dom}(\delta)$. Therefore, for any $F \in \mathbb{D}_{1,2}$ and any $u \in \operatorname{Dom}(\delta)$ we have

$$
E[F \delta(u)]=E\left[\langle D F, u\rangle_{H}\right]
$$

We also set

$$
\operatorname{Dom}(L)=\left\{F \in L^{2}(W): \sum_{q=1}^{\infty} q^{2} E\left[\left\|J_{q} F\right\|^{2}\right]<\infty\right\} .
$$

Then the Ornstein-Uhlenbeck operator $L: \operatorname{Dom}(L) \rightarrow L^{2}(W)$ is defined by

$$
L F=-\sum_{q=1}^{\infty} q J_{q} F, \quad F \in \operatorname{Dom}(L),
$$

where the convergence of the series is considered in $L^{2}(W)$. It is well-known that $F \in L^{2}(W)$ belongs to $\operatorname{Dom}(L)$ if and only if $F \in \mathbb{D}_{1,2}$ and $D F \in \operatorname{Dom}(\delta)$, and this case we have $L F=-\delta D F$ (cf. Proposition 1.4.3 of [52]). Finally, the pseudo inverse of $L$, denoted by $L^{-1}$, is the operator on $L^{2}(W)$ into $\operatorname{Dom}(L)$ defined by

$$
L^{-1} F=-\sum_{q=1}^{\infty} \frac{1}{q} J_{q} F, \quad F \in L^{2}(W) .
$$

It holds that $L L^{-1} F=F-E[F]$ for all $F \in L^{2}(W)$ (cf. Proposition 2.8.11 of [48]).

\section{A.2 Technical tools from the Chernozhukov-Chetverikov-Kato theory}

This subsection collects the technical results of the Chernozhukov-Chetverikov-Kato theory, which are used in this paper. The first result is a corollary of Lemmas 3-4 of [15] and Lemma 4.3 of [13]:

Lemma A.1. Let $g: \mathbb{R} \rightarrow \mathbb{R}$ be a $C^{2}$ function. Then we have

$$
\sum_{i, j=1}^{d}\left|\frac{\partial^{2}\left(g \circ \Phi_{\beta}\right)}{\partial x_{i} \partial x_{j}}(x)\right| \leq\left\|g^{\prime \prime}\right\|_{\infty}+2 \beta\left\|g^{\prime}\right\|_{\infty}
$$

for all $x \in \mathbb{R}^{d}$. Moreover, if $g$ is a $C^{3}$ function, we also have

$$
\sum_{i, j, k=1}^{d}\left|\frac{\partial^{3}\left(g \circ \Phi_{\beta}\right)}{\partial x_{i} \partial x_{j} \partial x_{k}}(x)\right| \leq\left\|g^{\prime \prime \prime}\right\|_{\infty}+6 \beta\left\|g^{\prime \prime}\right\|_{\infty}+6 \beta^{2}\left\|g^{\prime}\right\|_{\infty}
$$

for all $x \in \mathbb{R}^{d}$.

Proof. The first inequality is a direct consequence of Lemmas 3-4 from [15]. The second inequality is Eq.(19) in Lemma 4.3 of [13].

The next result is taken from Lemma 5.1 of [16]:

Lemma A.2 ([16], Lemma 5.1). For any $\varepsilon>0$ and any Borel set $A$ of $\mathbb{R}$, there is a $C^{\infty}$ function $g: \mathbb{R} \rightarrow \mathbb{R}$ satisfying the following conditions: 
(i) There is a universal constant $K>0$ such that $\left\|g^{\prime}\right\|_{\infty} \leq \varepsilon^{-1}$, $\left\|g^{\prime \prime}\right\|_{\infty} \leq K \varepsilon^{-2}$ and $\left\|g^{\prime \prime \prime}\right\|_{\infty} \leq K \varepsilon^{-3}$.

(ii) $1_{A}(x) \leq g(x) \leq 1_{A^{3 \varepsilon}}(x)$ for all $x \in \mathbb{R}$.

The third one is a corollary of Lemma 4.1 from [13] and Lemma 2.1 from [16]:

Lemma A.3. Let $V, W$ be random variables. Suppose that there are constants $r_{1}, r_{2}>0$ such that

$$
P(V \in A) \leq P\left(W \in A^{r_{1}}\right)+r_{2}
$$

for any Borel set $A$ of $\mathbb{R}$. Then we have

$$
\sup _{x \in \mathbb{R}}|P(V \leq x)-P(W \leq x)| \leq \sup _{x \in \mathbb{R}} P\left(|W-x| \leq r_{1}\right)+r_{2} .
$$

Proof. By extending the probability space $(\Omega, \mathcal{F}, P)$ if necessary, we may assume that there is a uniform random variable on $(0,1)$ independent of $V$ without loss of generality. Then, by Lemma 4.1 from [13] there is a random variable $W^{\prime}$ whose distribution is the same as that of $W$ such that $P\left(\left|V-W^{\prime}\right|>r_{1}\right) \leq r_{2}$. Therefore, the desired result follows from Lemma 2.1 of [15].

The fourth result is a so-called anti-concentration inequality for maxima of Gaussian variables, which is taken from Theorem 3 of [15]:

Lemma A.4 ([15], Theorem 3). Let $X=\left(X_{1}, \ldots, X_{d}\right)^{\top}$ be a d-dimensional centered Gaussian random vector with $\sigma_{j}^{2}:=E\left[X_{j}^{2}\right]>0$ for $j=1, \ldots, d$. Suppose that there are two constants $\bar{\sigma}, \underline{\sigma}>0$ such that $\underline{\sigma} \leq \sigma_{j} \leq \bar{\sigma}$ for all $j=1, \ldots, d$. Then, there is a constant $C>0$ which only depends on $\bar{\sigma}$ and $\underline{\sigma}$ such that

$$
\sup _{x \in \mathbb{R}} P\left(\left|X_{\vee}-x\right| \leq \varepsilon\right) \leq C \varepsilon\left\{a_{d}+\sqrt{1 \vee \log (\underline{\sigma} / \varepsilon)}\right\}
$$

for any $\varepsilon>0$, where $a_{d}=E\left[\max _{1 \leq j \leq d}\left(X_{j} / \sigma_{j}\right)\right]$.

The fifth result is another anti-concentration inequality for maxima of Gaussian variables, which is taken from Lemma 4.3 of [16]:

Lemma A.5 ([16], Lemma 4.3). Let $X=\left(X_{1}, \ldots, X_{d}\right)^{\top}$ be a (possibly uncentered) d-dimensional Gaussian random vector such that $\operatorname{Var}\left[X_{j}\right]>0$ for all $j=1, \ldots, d$. Then for every $\varepsilon>0$,

$$
\sup _{x \in \mathbb{R}} P\left(\left|X_{\vee}-x\right| \leq \varepsilon\right) \leq \frac{2}{\underline{\sigma}} \varepsilon(\sqrt{2 \log d}+2),
$$

where $\underline{\sigma}=\min _{1 \leq j \leq d} \sqrt{\operatorname{Var}\left[X_{j}\right]}$.

The last result is an anti-concentration inequality for supremum of a Gaussian process taken from Corollary 2.1 of [12]:

Lemma A.6 ([12], Corollary 2.1). Let $X=\left(X_{t}\right)_{t \in \mathbb{T}}$ be a separable Gaussian process indexed by a semimetric space $\mathbb{T}$ such that $E\left[X_{t}\right]=0$ and $E\left[X_{t}^{2}\right]=1$ for all $t \in \mathbb{T}$. Assume that $\sup _{t \in \mathbb{T}} X_{t}<\infty$ a.s. Then $E\left[\sup _{t \in \mathbb{T}}\left|X_{t}\right|\right]<\infty$ and

$$
\sup _{x \in \mathbb{R}} P\left(\left|\sup _{t \in \mathbb{T}}\right| X_{t}|-x| \leq \varepsilon\right) \leq 4 \varepsilon\left(E\left[\sup _{t \in \mathbb{T}}\left|X_{t}\right|\right]+1\right)
$$

for all $\varepsilon \geq 0$. 


\section{A.3 Sub-Gaussian chaos property}

This subsection presents the notion of sub-Gaussian chaos property of random variables and stochastic processes, which is introduced in $[60,61]$ and serves as deriving maximal inequalities used in this paper.

Definition A.1 ([61], Definition 4.1; [60], Definition 2.1). Let $q$ be a positive integer. A (possibly uncentered) random variable $Y$ is said to have the sub-qth-Gaussian chaos property (or is a sub-qth chaos random variable, or is a sub-Gaussian chaos random variable of order $q$, etc.) relative to the scale $M \geq 0$ if

$$
E\left[\exp \left(\left(\frac{|Y|}{M}\right)^{2 / q}\right)\right] \leq 2
$$

Here, when $M=0$, for $x \geq 0, x / M$ is understood to be 0 if $x=0$ and $\infty$ otherwise. Hence, $Y$ is a sub- $q$ th chaos random variable relative to the scale 0 if and only if $Y=0$ a.s.

Note that, unlike [60, 61], we also allow sub-Gaussian chaos random variables to be uncentered. The following result is a useful criterion for this property.

Lemma A.7. For any positive integer $q$ and constant $C>0$, there is a positive number $M$ (which depends only on $q$ and $C$ ) such that any random variable $Y$, which satisfies $\|Y\|_{p} \leq C p^{q / 2} \Lambda$ for some constant $\Lambda \geq 0$ and any positive integer $p$, is a sub-qth chaos random variable relative to the scale $M \Lambda$.

Proof. For any $M>0$ we have

$$
E\left[\exp \left(\left(\frac{|Y|}{M \Lambda}\right)^{2 / q}\right)\right]=\sum_{k=0}^{\infty} \frac{1}{k !} E\left[\left(\frac{|Y|}{M \Lambda}\right)^{2 k / q}\right] \leq 1+\sum_{k=1}^{\infty} C^{2 k / q} \frac{(2 k / q)^{k}}{k ! M^{2 k / q}}
$$

Set $a_{k}(M)=C^{2 k / q}(2 k / q)^{k} / k ! M^{2 k / q}$. Then we have

$$
\frac{a_{k+1}(M)}{a_{k}(M)}=\frac{C^{2 / q}}{M^{2 / q}}\left(\frac{2 k+2}{2 k}\right)^{k} \frac{2 k+2}{q(k+1)} \rightarrow \frac{C^{2 / q}}{M^{2 / q}} \cdot e \cdot \frac{2}{q}
$$

as $k \rightarrow \infty$. Therefore, for sufficiently large $M$ we have $\lim _{k \rightarrow \infty} a_{k+1}(M) / a_{k}(M)<1$, hence we obtain $\sum_{k=1}^{\infty} a_{k}(M)<\infty$ by the d'Alembert ratio test. Since $a_{k}(M)$ is a decreasing function of $M>0$ for all $k \in \mathbb{N}$, the dominated convergence theorem yields $\sum_{k=1}^{\infty} a_{k}(M) \rightarrow 0$ as $M \rightarrow \infty$. Therefore, there is a constant $M>0$ which depends only on $C$ and $q$ such that $\sum_{k=1}^{\infty} a_{k}(M) \leq 1$. For this $M$ the claim of the lemma holds true.

The condition on $Y$ in Lemma A.7 is typically satisfied with $\Lambda=\|Y\|_{2}$. In particular, combining Lemma A.7 with Theorem 5.11 and Remark 5.11 from [32], we obtain the following result.

Proposition A.1. For any positive integer $q$, there is a constant $M>0$ which depends only on $q$ such that, for any Gaussian Hilbert space $\mathbb{H}, Y$ is a sub-qth chaos random variable relative to the scale $M\|Y\|_{2}$ for all $Y \in \overline{\mathcal{P}}_{q}(\mathbb{H})$.

The next result presents maximal moment inequalities obtained from the sub-Gaussian chaos property.

Proposition A.2. For each $k=1, \ldots, d$, let $Y_{k}$ be a sub-qth chaos random variable relative to the scale $M_{k} \geq 0$.

Then we have

$$
E\left[\max _{1 \leq k \leq d}\left|Y_{k}\right|^{p}\right] \leq\left(\max _{1 \leq k \leq d} M_{k}^{p}\right) \log ^{p q / 2}\left(2 d-1+e^{p q / 2-1}\right)
$$

for any $p>0$ such that $p q \geq 2$. 
Proof. By Lemma 14.7 from [7] we have

$$
E\left[\max _{1 \leq k \leq d}\left|Y_{k}\right|^{p}\right] \leq\left(\max _{1 \leq k \leq d} M_{k}^{p}\right) \log ^{p q / 2}\left(E\left[\exp \left(\max _{1 \leq k \leq d}\left(\frac{\left|Y_{k}\right|}{M_{k}}\right)^{2 / q}\right)\right]-1+e^{p q / 2-1}\right) .
$$

Since we have

$$
E\left[\exp \left(\max _{1 \leq k \leq d}\left(\frac{\left|Y_{k}\right|}{M_{k}}\right)^{2 / q}\right)\right]=E\left[\max _{1 \leq k \leq d} \exp \left(\left(\frac{\left|Y_{k}\right|}{M_{k}}\right)^{2 / q}\right)\right] \leq \sum_{k=1}^{d} E\left[\exp \left(\left(\frac{\left|Y_{k}\right|}{M_{k}}\right)^{2 / q}\right)\right] \leq 2 d,
$$

we obtain the desired result.

To conclude this subsection, we present an estimate for the modulus of continuity for sub-Gaussian chaos processes, which is established in [60]. In the following, $(\mathbb{T}, \mathfrak{d})$ denotes a semi-metric space.

Definition A.2 ([61], Definition 4.3; [60], Definition 2.3). Let $q$ be a positive integer. A centered real-valued process $X=\left(X_{t}\right)_{t \in \mathbb{T}}$ is said to be a sub-qth-Gaussian chaos process with respect to $\mathfrak{d}$ if the random variable $X(t)-X(s)$ has the sub- $q$ th-Gaussian chaos property relative to the scale $\mathfrak{d}(s, t)$ for any $s, t \in \mathbb{T}$.

Definition A.3. Let $r$ be a positive number. An $r$-covering number of $\mathbb{T}$ with respect to $\mathfrak{d}$, which is denoted by $N(\mathbb{T}, \mathfrak{d}, r)$, is the smallest positive integer $N$ such that there are points $t_{1}, \ldots, t_{N} \in \mathbb{T}$ satisfying for any $t \in \mathbb{T}$ there is an index $i \in\{1, \ldots, N\}$ with $\mathfrak{d}\left(t, t_{i}\right)<r$ (we set $N(\mathbb{T}, \mathfrak{d}, r)=\infty$ if there is no such an $N$ ).

The following result follows from Remark 2.2, Corollary 3.2 and the discussion at the beginning of Section 5 from [60]:

Proposition A.3. Let $q$ be a positive integer. There is a number $C_{q}>0$ which depends only on $q$ such that the variable $\sup _{s, t \in \mathbb{T}: \mathfrak{o}(s, t) \leq \eta}\left|X_{s}-X_{t}\right|$ is a sub-qth chaos random variable relative to the scale

$$
C_{q} \int_{0}^{\eta} \log ^{q / 2} N(\mathbb{T}, \mathfrak{d}, r) d r
$$

for any $\eta>0$ and any separable centered sub-qth-Gaussian chaos process $X=\left(X_{t}\right)_{t \in \mathbb{T}}$ with respect to $\mathfrak{d}$.

Remark A.1. For the case $q=1$ or $q=2$, related estimates to Proposition A.3 can be found in Section 2.2 of [59] (they are indeed sufficient for this paper). We also remark that Dirksen [22] gives a shaper estimate than Proposition A.3 for general values of $q$ (see Theorem 3.2 and Remark 3.3 of [22] for details).

\section{B Proofs}

\section{B.1 Proof of Proposition 2.1}

The proof of Proposition 2.1 relies on the following integration by parts formula established by Nourdin and Peccati:

Lemma B.1 (Nordin-Peccati's formula). Let $G \in L^{2}(W)$ and $\psi: \mathbb{R}^{d} \rightarrow \mathbb{R}$ be a $C^{1}$ function with bounded partial derivatives. Then we have

$$
\operatorname{Cov}[\psi(F), G]=\sum_{i=1}^{d} E\left[\frac{\partial \psi}{\partial x_{i}}(F)\left\langle D F_{i},-D L^{-1} G\right\rangle_{H}\right] .
$$

This lemma is a straightforward extension of Theorem 2.9.1 from [48], so we omit its proof. 
Proof of Proposition 2.1. The latter claim immediately follows from the former and (2.1). To prove the former claim, we may assume that $F$ and $Z$ are independent without loss of generality. Let us set $\varphi:=g \circ \Phi_{\beta}$ and define the function $\Psi:[0,1] \rightarrow \mathbb{R}$ by $\Psi(t)=E[\varphi(\sqrt{t} F+\sqrt{1-t} Z)], t \in[0,1]$. We can easily check that $\Psi$ is continuous on $[0,1]$ and differentiable on $(0,1)$, and we have

$$
\Psi^{\prime}(t)=\frac{1}{2} \sum_{j=1}^{d} E\left[\frac{\partial \varphi}{\partial x_{j}}(\sqrt{t} F+\sqrt{1-t} Z)\left(\frac{F_{j}}{\sqrt{t}}-\frac{Z_{j}}{\sqrt{1-t}}\right)\right]
$$

for every $t \in(0,1)$. Now, by Lemma A.1 we have

$$
\sum_{i, j=1}^{d}\left|\frac{\partial^{2} \varphi}{\partial x_{i} x_{j}}(x)\right| \leq\left\|g^{\prime \prime}\right\|_{\infty}+2 \beta\left\|g^{\prime}\right\|_{\infty}
$$

for any $x \in \mathbb{R}^{d}$. In particular, $\partial^{2} \varphi / \partial x_{i} \partial x_{j}$ is bounded for all $i, j=1, \ldots, d$. Therefore, noting that the independence between $Z$ and $F$, Stein's identity (e.g. Lemma 2 of [15]) implies that

$$
\sum_{j=1}^{d} E\left[\frac{\partial \varphi}{\partial x_{j}}(\sqrt{t} F+\sqrt{1-t} Z) \frac{Z_{j}}{\sqrt{1-t}}\right]=\sum_{i, j=1}^{d} E\left[\frac{\partial^{2} \varphi}{\partial x_{i} \partial x_{j}}(\sqrt{t} F+\sqrt{1-t} Z) \mathfrak{C}(i, j)\right] .
$$

Moreover, Nourdin-Peccati's formula (Lemma B.1) yields

$$
\sum_{j=1}^{d} E\left[\frac{\partial \varphi}{\partial x_{j}}(\sqrt{t} F+\sqrt{1-t} Z) \frac{F_{j}}{\sqrt{t}}\right]=\sum_{i, j=1}^{d} E\left[\frac{\partial^{2} \varphi}{\partial x_{i} \partial x_{j}}(\sqrt{t} F+\sqrt{1-t} Z)\left\langle D F_{i},-D L^{-1} F_{j}\right\rangle_{H}\right] .
$$

Hence we conclude that

$$
\Psi^{\prime}(t)=\frac{1}{2} \sum_{i, j=1}^{d} E\left[\frac{\partial^{2} \varphi}{\partial x_{i} \partial x_{j}}(\sqrt{t} F+\sqrt{1-t} Z)\left(\left\langle D F_{i},-D L^{-1} F_{j}\right\rangle_{H}-\mathfrak{C}(i, j)\right)\right]
$$

for every $t \in(0,1)$. Consequently, we obtain

$$
|E[\varphi(F)]-E[\varphi(Z)]| \leq \int_{0}^{1}\left|\Psi^{\prime}(t)\right| d t \leq\left(\left\|g^{\prime \prime}\right\|_{\infty} / 2+\beta\left\|g^{\prime}\right\|_{\infty}\right) \Delta,
$$

which completes the proof.

\section{B.2 Proof of Lemma 2.1}

Let us set $\beta=\varepsilon^{-1} \log d$ (hence $\beta^{-1} \log d=\varepsilon$ ). From (2.1) we have

$$
P\left(F_{\vee} \in A\right) \leq P\left(\Phi_{\beta}(F) \in A^{\varepsilon}\right)=E\left[1_{A^{\varepsilon}}\left(\Phi_{\beta}(F)\right)\right] .
$$

Next, by Lemma A.2 there is a $C^{\infty}$ function $g: \mathbb{R} \rightarrow \mathbb{R}$ and a universal constant $K>0$ such that $\left\|g^{\prime}\right\|_{\infty} \leq$ $\varepsilon^{-1},\left\|g^{\prime \prime}\right\|_{\infty} \leq K \varepsilon^{-2},\left\|g^{\prime \prime \prime}\right\|_{\infty} \leq K \varepsilon^{-3}$ and $1_{A^{\varepsilon}}(x) \leq g(x) \leq 1_{A^{4 \varepsilon}}(x)$ for any $x \in \mathbb{R}$. Then we obtain $E\left[1_{A^{\varepsilon}}\left(\Phi_{\beta}(F)\right)\right] \leq E\left[g\left(\Phi_{\beta}(F)\right)\right]$. Now, by Proposition 2.1 we have

$$
\left|E\left[g\left(\Phi_{\beta}(F)\right)\right]-E\left[g\left(\Phi_{\beta}(Z)\right)\right]\right| \leq C_{1}\left(\varepsilon^{-2}+\varepsilon^{-2} \log d\right) \Delta \leq C_{2} \varepsilon^{-2}(\log d) \Delta,
$$

where $C_{1}, C_{2}>0$ denote universal constants. Moreover, we also have

$$
E\left[g\left(\Phi_{\beta}(Z)\right)\right] \leq E\left[1_{A^{4 \varepsilon}}\left(\Phi_{\beta}(Z)\right)\right] \leq E\left[1_{A^{5 \varepsilon}}\left(Z_{\vee}\right)\right]=P\left(Z_{\vee} \in A^{5 \varepsilon}\right) .
$$

This completes the proof. 


\section{B.3 Proof of Theorem 2.1}

If $\Delta \geq 1$, (2.2)-(2.3) hold true by taking $C=C^{\prime}=2$ (note that $d \geq 2$ ). Therefore, it suffices to consider the case of $\Delta<1$.

By Lemmas 2.1 and A.3 there is a universal constant $C_{0}>0$ such that

$$
\sup _{x \in \mathbb{R}}\left|P\left(F_{\vee} \leq x\right)-P\left(Z_{\vee} \leq x\right)\right| \leq \sup _{x \in \mathbb{R}} P\left(\left|Z_{\vee}-x\right| \leq 5 \varepsilon\right)+C_{0} \varepsilon^{-2}(\log d) \Delta .
$$

Now, under the assumptions of claim (a), Lemma A.4 yields

$$
\sup _{x \in \mathbb{R}} P\left(\left|Z_{\vee}-x\right| \leq 5 \varepsilon\right) \leq C_{1} \varepsilon\left\{a_{d}+\sqrt{1 \vee \log (\underline{\sigma} / \varepsilon)}\right\} \leq C_{2} \varepsilon \sqrt{1 \vee a_{d}^{2} \vee \log (1 / \varepsilon)},
$$

where $C_{1}, C_{2}>0$ depend only on $\underline{\sigma}$ and $\bar{\sigma}$, while we have

$$
\sup _{x \in \mathbb{R}} P\left(\left|Z_{\vee}-x\right| \leq 5 \varepsilon\right) \leq C_{1} \varepsilon \sqrt{\log d}
$$

where $C_{3}>0$ depends only on $b$, under the assumptions of claim (b) due to Lemma A.5. Consequently, if $\Delta=0$, letting $\varepsilon$ tend to 0 , we obtain the desired results. Otherwise, take

$$
\varepsilon=\Delta^{1 / 3}\left(1 \vee a_{d} \vee \log ^{1 / 2}(1 / \Delta)\right)^{-1 / 3}(2 \log d)^{1 / 3}
$$

for claim (a). Then the same argument as the one in the proof of Theorem 2 from [15] yields (2.2). For claim (b), taking $\varepsilon=\Delta^{1 / 3}(\log d)^{1 / 6}$, we obtain (2.3).

\section{B.4 Proof of Lemma 2.2}

By the triangular inequality it suffices to prove

$$
E\left[\max _{1 \leq i, j \leq d}\left|\Delta_{i, j}\right|\right] \leq C_{q} \log ^{q-1}\left(2 d^{2}-1+e^{q-2}\right) \max _{1 \leq k \leq d} \sqrt{E\left[F_{k}^{4}\right]-3 E\left[F_{k}^{2}\right]^{2}}
$$

where $\Delta_{i, j}=E\left[F_{i} F_{j}\right]-\left\langle D F_{i},-D L^{-1} F_{j}\right\rangle_{H}$. From the proof of Lemma 6.2.1 from [48] we deduce that

$$
\left\langle D F_{i}, D F_{j}\right\rangle_{H}=q^{2} \sum_{r=1}^{q}(r-1) !\left(\begin{array}{c}
q-1 \\
r-1
\end{array}\right)^{2} I_{2 q-2 r}\left(f_{i} \widetilde{\otimes}_{r} f_{j}\right)
$$

Since $-L^{-1} F_{j}=q^{-1} F_{j}$, we obtain

$$
\Delta_{i, j}=-q \sum_{r=1}^{q-1}(r-1) !\left(\begin{array}{c}
q-1 \\
r-1
\end{array}\right)^{2} I_{2 q-2 r}\left(f_{i} \widetilde{\otimes}_{r} f_{j}\right) .
$$

In particular, by Proposition A.1 there is a constant $M_{q}>0$ which only depends on $q$ such that $\Delta_{i, j}$ is a sub$(2 q-2)$ th chaos random variable relative to the scale $M_{q}\left\|\Delta_{i, j}\right\|_{2}$. Therefore, by Proposition A.2 we have

$$
E\left[\max _{1 \leq i, j \leq d}\left|\Delta_{i, j}\right|\right] \leq M_{q} \log ^{q-1}\left(2 d^{2}-1+e^{q-2}\right) \max _{1 \leq k \leq d}\left\|\Delta_{i, j}\right\|_{2} .
$$

Therefore, the proof is completed once we show that

$$
\max _{1 \leq i, j \leq d} E\left[\Delta_{i, j}^{2}\right] \leq\left\{\sum_{r=1}^{q-1}\left(\begin{array}{c}
2 r \\
r
\end{array}\right)\right\} \max _{1 \leq k \leq d}\left(E\left[F_{k}^{4}\right]-3 E\left[F_{k}^{2}\right]^{2}\right),
$$

which follows from the equation $\Delta_{i, j}=E\left[F_{i} F_{j}\right]-q^{-1}\left\langle D F_{i}, D F_{j}\right\rangle_{H}$ and Eq.(6.2.6) of [48]. 


\section{B.5 Proof of Lemma 2.3}

Lemma B.2. If $F_{1}, \ldots, F_{d} \in \mathbb{D}_{2,4 p}$ for a positive integer $p$, we have

$$
\begin{aligned}
\max _{1 \leq i, j \leq d}\left\|E\left[F_{i} F_{j}\right]-\left\langle D F_{i},-D L^{-1} F_{j}\right\rangle_{H}\right\|_{2 p} & \\
& \leq \sqrt{2 p-1} \cdot \frac{3}{2}\left(\max _{1 \leq i \leq d}\|\| D^{2} F_{i}\left\|_{o p}\right\|_{4 p}\right)\left(\max _{1 \leq j \leq d}\|\| D F_{j}\left\|_{H}\right\|_{4 p}\right) .
\end{aligned}
$$

Proof. For any $i, j=1, \ldots, d$, we have $E\left[\left\langle D F_{i},-D L^{-1} F_{j}\right\rangle_{H}\right]=E\left[F_{i} F_{j}\right]$ by Nourdin-Peccati's formula. Therefore, it holds that

$$
\begin{aligned}
& \left\|E\left[F_{i} F_{j}\right]-\left\langle D F_{i},-D L^{-1} F_{j}\right\rangle_{H}\right\|_{2 p} \\
& \leq \sqrt{2 p-1}\left\|D\left\langle D F_{i},-D L^{-1} F_{j}\right\rangle_{H}\right\|_{2 p}(\because \text { Lemma } 5.3 .7 \text { of [48] }) \\
& =\sqrt{2 p-1}\left\|\left\langle D^{2} F_{i},-D L^{-1} F_{j}\right\rangle_{H}+\left\langle D F_{i},-D^{2} L^{-1} F_{j}\right\rangle_{H}\right\|_{2 p}(\because \text { Lemma } 5.3 .8 \text { of [48] }) \\
& \leq \sqrt{2 p-1}\left(\left\|\left\langle D^{2} F_{i},-D L^{-1} F_{j}\right\rangle_{H}\right\|_{2 p}+\left\|\left\langle D F_{i},-D^{2} L^{-1} F_{j}\right\rangle_{H}\right\|_{2 p}\right) \\
& \leq \sqrt{2 p-1}\left(\|\| D^{2} F_{i}\left\|_{\mathrm{op}}\right\| D L^{-1} F_{j}\left\|_{H}\right\|_{2 p}+\|\| D F_{i}\left\|_{H}\right\| D^{2} L^{-1} F_{j}\left\|_{\mathrm{op}}\right\|_{2 p}\right) \\
& \leq \sqrt{2 p-1}\left(\|\| D^{2} F_{i}\left\|_{\mathrm{op}}\right\|_{4 p}\|\| D L^{-1} F_{j}\left\|_{H}\right\|_{4 p}+\|\| D F_{i}\left\|_{H}\right\|_{4 p}\|\| D^{2} L^{-1} F_{j}\left\|_{\mathrm{op}}\right\|_{4 p}\right)(\because \text { the Schwarz inequality }) \\
& \leq \sqrt{2 p-1}\left(\|\| D^{2} F_{i}\left\|_{\mathrm{op}}\right\|\left\|_{4 p}\right\|\left\|D F_{j}\right\|_{H}\left\|_{4 p}+\frac{1}{2}\right\|\left\|D F_{i}\right\|_{H}\left\|_{4 p}\right\|\left\|D^{2} F_{j}\right\|_{\mathrm{op}} \|_{4 p}\right)(\because \text { Lemma 5.3.7 of [48]) } \\
& \leq \sqrt{2 p-1} \cdot \frac{3}{2}\left(\max _{1 \leq i \leq d}\|\| D^{2} F_{i}\left\|_{\mathrm{op}}\right\|_{4 p}\right)\left(\max _{1 \leq j \leq d}\|\| D F_{j}\left\|_{H}\right\|_{4 p}\right)
\end{aligned}
$$

This completes the proof.

Proof of Lemma 2.3. Since we have

$$
\begin{aligned}
& E\left[\max _{1 \leq i, j \leq d}\left|E\left[F_{i} F_{j}\right]-\left\langle D F_{i},-D L^{-1} F_{j}\right\rangle_{H}\right|\right] \\
& \leq\left\|\max _{1 \leq i, j \leq d}\left|E\left[F_{i} F_{j}\right]-\left\langle D F_{i},-D L^{-1} F_{j}\right\rangle_{H}\right|\right\|_{2 p} \\
& \leq d^{1 / p} \max _{1 \leq i, j \leq d}\left\|E\left[F_{i} F_{j}\right]-\left\langle D F_{i},-D L^{-1} F_{j}\right\rangle_{H} \mid\right\|_{2 p},
\end{aligned}
$$

the first inequality follows from Lemma B.2. Next, if both the variables $\left\|D^{2} F_{i}\right\|_{\text {op }}$ and $\left\|D F_{i}\right\|_{H}$ are sub-Gaussian relative to the scale $a$ for all $i=1, \ldots, d$, we have

$$
\begin{aligned}
\left\|E\left[F_{i} F_{j}\right]-\left\langle D F_{i},-D L^{-1} F_{j}\right\rangle_{H}\right\|_{p} & \leq\left\|E\left[F_{i} F_{j}\right]-\left\langle D F_{i},-D L^{-1} F_{j}\right\rangle_{H}\right\|_{2 p} \\
& \leq C_{0} p^{3 / 2} a^{2}
\end{aligned}
$$

with some universal constant $C_{0}>0$ for all $i, j=1, \ldots, d$ and any integer $p \geq 1$ by Lemma B.2 and Lemma 1 of [8]. Therefore, by Lemma A.7 there is another universal constant $C>0$ such that $E\left[F_{i} F_{j}\right]-\left\langle D F_{i},-D L^{-1} F_{j}\right\rangle_{H}$ is a sub-3rd chaos random variable relative to the scale $C a^{2}$. Now the second inequality of the lemma follows from Proposition A.2. 


\section{B.6 Proof of Theorem 3.1}

Without loss of generality we may assume that $\boldsymbol{\xi}_{n}$ can be written as $\boldsymbol{\xi}_{n}=\Sigma_{n}^{1 / 2} \boldsymbol{\eta}_{n}$, where $\boldsymbol{\eta}_{n}$ is an $N_{n^{-}}$ dimensional standard normal variable. Set $H_{n}=\mathbb{R}^{N_{n}}$ and set $W_{n}(h)=h^{\top} \boldsymbol{\eta}_{n}$ for $h \in H_{n}$. Then, $W_{n}=$ $\left(W_{n}(h)\right)_{h \in H_{n}}$ is an isonormal Gaussian process over $H_{n}$ and we have $\boldsymbol{\eta}_{n}=\left(W_{n}\left(e_{1}\right), \ldots, W_{n}\left(e_{N_{n}}\right)\right)^{\top}$, where $\left(e_{1}, \ldots, e_{N_{n}}\right)$ is the canonical basis of $H_{n}$.

Now let us denote by $\gamma_{n, k}(i, j)$ the $(i, j)$-th component of the $N_{n} \times N_{n}$ matrix $\Sigma_{n}^{1 / 2} A_{n . k} \Sigma_{n}^{1 / 2}$. Then by the product formula for multiple Wiener-Itô integrals (e.g. Theorem 2.7.10 of [48]) we can rewrite $F_{n, k}$ as

$$
F_{n, k}=\sum_{i, j=1}^{N_{n}} \gamma_{n, k}(i, j) I_{2}^{W_{n}}\left(e_{i} \otimes e_{j}\right)=I_{2}^{W_{n}}\left(f_{n, k}\right)
$$

where $I_{2}^{W_{n}}(\cdot)$ denotes the double Wiener-Itô integral with respect to $W_{n}$ and

$$
f_{n, k}=\sum_{i, j=1}^{N} \gamma_{n, k}(i, j) e_{i} \otimes e_{j}
$$

Therefore, by applying Theorem 2.1, Corollary 2.1 and Lemma 2.2, we obtain the desired result.

\section{B.7 Proof of Theorem 3.2}

We first derive some non-asymptotic results. For each $k=1, \ldots, d$, let $\Gamma_{k}=\left(\gamma_{k}(i, j)\right)_{1 \leq i, j \leq N}$ be an $N \times N$ symmetric matrix such that $\gamma_{k}(i, i)=0$ for every $i=1, \ldots, N$. Given a sequence $\xi=\left(\xi_{i}\right)_{i=1}^{\infty}$ of random variables, we set

$$
Q_{k}(\xi)=\sum_{i, j=1}^{N} \gamma_{k}(i, j) \xi_{i} \xi_{j}, \quad k=1, \ldots, d
$$

and $Q(\xi)=\left(Q_{1}(\xi), \ldots, Q_{d}(\xi)\right)^{\top}$. Also, we set

$$
\begin{aligned}
& R_{1}=\sum_{i=1}^{N} E\left[\max _{1 \leq k \leq d}\left|\sum_{j=1}^{N} \gamma_{k}(i, j) W_{j}^{(i)}\right|^{3}\right]\left(E\left[\left|Y_{i}\right|^{3}\right]+E\left[\left|G_{i}\right|^{3}\right]\right), \\
& R_{2}=\max _{1 \leq k, l \leq d}\left|\mathfrak{C}(k, l)-E\left[Q_{k}(G) Q_{l}(G)\right]\right| \\
& R_{3}=\max _{1 \leq k \leq d} \sqrt{E\left[Q_{k}(G)^{4}\right]-3 E\left[Q_{k}(G)^{2}\right]^{2}} .
\end{aligned}
$$

Lemma B.3. Let $g: \mathbb{R} \rightarrow \mathbb{R}$ be a $C^{3}$ function with bounded derivatives up to the third order. For any $\beta>0$ we have

$$
\left|E\left[g\left(\Phi_{\beta}(Q(Y))\right)\right]-E\left[g\left(\Phi_{\beta}(Q(G))\right)\right]\right| \leq \frac{4}{3}\left(\left\|g^{\prime \prime \prime}\right\|_{\infty}+6\left\|g^{\prime \prime}\right\|_{\infty} \beta+6\left\|g^{\prime}\right\|_{\infty} \beta^{2}\right) R_{1} .
$$

Proof. The proof is based on the generalized Lindeberg method developed in [43,50]. We start with introducing some notation following the proof of Proposition 11.4.2 from [48]. For $p=1, \ldots, d$ and $i=1, \ldots, N$, we define the variables $U_{p, i}$ and $V_{p, i}$ by

$$
U_{p, i}=\sum_{\substack{j, k=1 \\ j \neq i, k \neq i}}^{N} \gamma_{p}(j, k) W_{j}^{(i)} W_{k}^{(i)}, \quad V_{p, i}=2 \sum_{j=1}^{N} \gamma_{p}(i, j) W_{j}^{(i)}
$$


and set $U_{i}=\left(U_{p, i}\right)_{p=1}^{d}, V_{i}=\left(V_{p, i}\right)_{p=1}^{d}$. By construction both $Y_{i}$ and $G_{i}$ are independent of $\left(U_{i}, V_{i}\right)$ (note that $\gamma_{p}(i, i)=0$ for every $p$ ), and we have

$$
U_{p, i}+Y_{i} V_{p, i}=Q_{p}\left(W^{(i)}\right), \quad U_{p, i}+G_{i} V_{p, i}=Q_{p}\left(W^{(i-1)}\right)
$$

Set $h=g \circ \Phi_{\beta}$. Noting that $E\left[Y_{i}\right]=E\left[G_{i}\right]=0$ and $E\left[Y_{i}^{2}\right]=E\left[G_{i}^{2}\right]=1$ as well as the independence between $Y_{i}, G_{i}$ and $\left(U_{i}, V_{i}\right)$, the Taylor theorem yields

$$
\begin{aligned}
E\left[h\left(U_{i}+\xi_{i} V_{i}\right)\right]= & \frac{1}{2} \sum_{p, q=1}^{d} E\left[\frac{\partial^{2} h}{\partial x_{p} \partial x_{q}}\left(U_{i}\right) V_{p, i} V_{q_{i}}\right] \\
& +\frac{1}{2} \sum_{p, q, r=1}^{d} \int_{0}^{1}(1-t)^{2} E\left[\frac{\partial^{3} h}{\partial x_{p} \partial x_{q} \partial x_{r}}\left(U_{i}+t \xi_{i} V_{i}\right) V_{p, i} V_{q, i} V_{r, i} \xi_{i}^{3}\right] d t
\end{aligned}
$$

when $\xi_{i}=Y_{i}$ or $\xi_{i}=G_{i}$. This implies that

$$
\begin{aligned}
& \left|E\left[h\left(Q\left(W^{(i)}\right)\right)\right]-E\left[h\left(Q\left(W^{(i-1)}\right)\right)\right]\right| \\
& \leq \frac{1}{2} \int_{0}^{1}(1-t)^{2} E\left[\sum_{p, q, r=1}^{d}\left|\frac{\partial^{3} h}{\partial x_{p} \partial x_{q} \partial x_{r}}\left(U_{i}+t Y_{i} V_{i}\right)\right| \max _{1 \leq p, q, r \leq d}\left|V_{p, i} V_{q, i} V_{r, i}\right|\left|Y_{i}\right|^{3}\right] d t \\
& \quad+\frac{1}{2} \int_{0}^{1}(1-t)^{2} E\left[\sum_{p, q, r=1}^{d}\left|\frac{\partial^{3} h}{\partial x_{p} \partial x_{q} \partial x_{r}}\left(U_{i}+t G_{i} V_{i}\right)\right| \max _{1 \leq p, q, r \leq d}\left|V_{p, i} V_{q, i} V_{r, i}\right|\left|G_{i}\right|^{3}\right] d t .
\end{aligned}
$$

Therefore, by Lemma A.1 we obtain

$$
\begin{aligned}
& \left|E\left[h\left(Q\left(W^{(i)}\right)\right)\right]-E\left[h\left(Q\left(W^{(i-1)}\right)\right)\right]\right| \\
& \leq \frac{1}{6}\left(\left\|g^{\prime \prime \prime}\right\|_{\infty}+6\left\|g^{\prime \prime}\right\|_{\infty} \beta+6\left\|g^{\prime}\right\|_{\infty} \beta^{2}\right) E\left[\max _{1 \leq p, q, r \leq d}\left|V_{p, i} V_{q, i} V_{r, i}\right|\left(\left|Y_{i}\right|^{3}+\left|G_{i}\right|^{3}\right)\right] \\
& \leq \frac{1}{6}\left(\left\|g^{\prime \prime \prime}\right\|_{\infty}+6\left\|g^{\prime \prime}\right\|_{\infty} \beta+6\left\|g^{\prime}\right\|_{\infty} \beta^{2}\right) E\left[\max _{1 \leq p \leq d}\left|V_{p, i}\right|^{3}\right]\left(E\left[\left|Y_{i}\right|^{3}\right]+E\left[\left|G_{i}\right|^{3}\right]\right),
\end{aligned}
$$

where we use the independence between $Y_{i}, G_{i}$ and $V_{i}$ as well as the inequality of arithmetic and geometric means to obtain the last inequality. Now, using the identity

$$
E[h(Q(Y))]-E[h(Q(G))]=\sum_{i=1}^{N}\left(E\left[h\left(Q\left(W^{(i)}\right)\right)\right]-E\left[h\left(Q\left(W^{(i-1)}\right)\right)\right]\right),
$$

we obtain the desired result.

Lemma B.4. For any $\varepsilon>0$ and any Borel set $A$ of $\mathbb{R}$, we have

$$
P\left(Q_{\vee}(Y) \in A\right) \leq P\left(Z_{\vee} \in A^{5 \varepsilon}\right)+C\left\{\varepsilon^{-3}\left(\log ^{2} d\right) R_{1}+\varepsilon^{-2}(\log d) R_{2}+\varepsilon^{-2}\left(\log ^{2} d\right) R_{3}\right\},
$$

where $C>0$ is a universal constant.

Proof. Let us set $\beta=\varepsilon^{-1} \log d$ (hence $\beta^{-1} \log d=\varepsilon$ ). From (2.1) we have

$$
P\left(Q_{\vee}(Y) \in A\right) \leq P\left(\Phi_{\beta}(Q(Y)) \in A^{\varepsilon}\right)=E\left[1_{A^{\varepsilon}}\left(\Phi_{\beta}(Q(Y))\right)\right]
$$


Next, by Lemma A.2 there is a $C^{\infty}$ function $g: \mathbb{R} \rightarrow \mathbb{R}$ and a universal constant $K>0$ such that $\left\|g^{\prime}\right\|_{\infty} \leq$ $\varepsilon^{-1},\left\|g^{\prime \prime}\right\|_{\infty} \leq K \varepsilon^{-2},\left\|g^{\prime \prime \prime}\right\|_{\infty} \leq K \varepsilon^{-3}$ and $1_{A^{\varepsilon}}(t) \leq g(t) \leq 1_{A^{4 \varepsilon}}(t)$ for any $t \in \mathbb{R}$. Then we obtain $E\left[1_{A^{\varepsilon}}\left(\Phi_{\beta}(Q(Y))\right)\right] \leq E\left[g\left(\Phi_{\beta}(Q(Y))\right)\right]$. Now, by Lemma B.3 we have

$$
\left|E\left[g\left(\Phi_{\beta}(Q(Y))\right)\right]-E\left[g\left(\Phi_{\beta}(Q(G))\right)\right]\right| \leq C_{1}\left(\varepsilon^{-3}+\varepsilon^{-3} \log d+\varepsilon^{-3} \log ^{2} d\right) R_{1} \leq C_{2} \varepsilon^{-3}\left(\log ^{2} d\right) R_{1},
$$

where $C_{1}, C_{2}>0$ denote universal constants. Moreover, as in the proof of Theorem 3.1, we can apply Proposition 2.1 as well as Lemma 2.2 to $Q(G)$ and thus obtain

$$
\left|E\left[g\left(\Phi_{\beta}(Q(G))\right)\right]-E\left[g\left(\Phi_{\beta}(Z)\right)\right]\right| \leq C_{3} \varepsilon^{-2}(\log d)\left\{R_{2}+(\log d) R_{3}\right\} .
$$

with some universal constant $C_{3}>0$. Since we have

$$
E\left[g\left(\Phi_{\beta}(Z)\right)\right] \leq E\left[1_{A^{4 \varepsilon}}\left(\Phi_{\beta}(Z)\right)\right] \leq E\left[1_{A^{5 \varepsilon}}\left(Z_{\vee}\right)\right]=P\left(Z_{\vee} \in A^{5 \varepsilon}\right)
$$

the proof is completed.

Proof of Theorem 3.2. By Lemmas B.4, A.3 and A.5, we obtain

$$
\begin{aligned}
& \sup _{x \in \mathbb{R}}\left|P\left(Q_{\vee}(Y) \leq x\right)-P\left(Z_{\vee} \leq x\right)\right| \\
& \leq C\left\{\varepsilon \sqrt{\log d_{n}}+\varepsilon^{-3}\left(\log ^{2} d_{n}\right) R_{n, 1}+\varepsilon^{-2}\left(\log d_{n}\right) R_{n, 2}+\varepsilon^{-2}\left(\log ^{2} d_{n}\right) R_{n, 3}\right\}
\end{aligned}
$$

where $C>0$ depends only on $b$. Therefore, taking $\varepsilon=R_{n, 1}^{1 / 4}\left(\log d_{n}\right)^{3 / 8} \vee R_{n, 2}^{1 / 3}\left(\log d_{n}\right)^{1 / 6} \vee R_{n, 3}^{1 / 3} \sqrt{\log d_{n}}$, we obtain the desired result.

\section{B.8 Proof of Lemma 3.1}

We first prove the claim (a). For the proof it is convenient to introduce the notion of the hypercontractivity of a random variable. Let us recall that a random variable $Y$ is said to be $(p, q, \eta)$-hypercontractive for $1 \leq p \leq q<\infty$ and $0<\eta<1$ if

$$
\|a+\eta Y\|_{q} \leq\|a+Y\|_{p}
$$

for all $a \in \mathbb{R}$. We refer to [37], [40] and [43] for more details on this notion. We note that, if $Y$ is $(p, q, \eta)$ hypercontractive and $E[Y]=0$, then $Y$ is $\left(p, q, \eta^{\prime}\right)$-hypercontractive for all $\eta^{\prime} \in(0, \eta)$ (this follows from Lemma 1.1 of [37]). Lemma 3.1 is obtained as a special case of the following result:

Lemma B.5. Suppose that there are numbers $p \in[3, \infty)$ and $\eta \in(0,1)$ such that $Y_{i}$ is $(2, p, \eta)$-hypercontractive for all $i=1, \ldots, N_{n}$. Then we have

$$
\sum_{i=1}^{N_{n}} E\left[\max _{1 \leq k \leq d_{n}}\left|\sum_{j=1}^{N_{n}} \gamma_{n, k}(i, j) W_{j}^{(i)}\right|^{3}\right] \leq d_{n}^{3 / p} \eta^{-3} \sum_{i=1}^{N_{n}} \max _{1 \leq k \leq d_{n}}\left(\sum_{j=1}^{N_{n}} \gamma_{n, k}(i, j)^{2}\right)^{3 / 2}
$$

Proof. First we note that $\eta \leq(p-1)^{-1 / 2}$ (see page 333 of [43]). Then, since $G_{i}$ is $\left(2, p,(p-1)^{-1 / 2}\right)^{-}$ hypercontractive for all $i$ by Corollary 3.4 .1 of $[40], W_{j}^{(i)}$ is $(2, p, \eta)$-hypercontractive for all $i, j$. Therefore, the desired inequality follows from the Lyapunov inequality and Remark 3.3.1 of [40].

Proof of Lemma 3.1(a). By Proposition 3.16 from [43], $Y_{i}$ is $\left(2, p,\left(2 \sqrt{p-1}\left\|Y_{i}\right\|_{p}\right)^{-1}\right)$-hypercontractive for all $i=1, \ldots, N_{n}$. Hence the desired result immediately follows from the above lemma. 
Next we turn to the claim (b). For the proof we use some elementary properties on the sub-Gaussian property which we enumerate for convenience. In this case we always have $E[Y]=0$ and $\operatorname{Var}[Y] \leq b^{2}$ (cf. Proposition 2.1 of [55]). A standard Gaussian variable is evidently sub-Gaussian relative to the scale 1 . If $Y_{1}, \ldots, Y_{m}$ are independent random variables and $Y_{i}$ is sub-Gaussian relative to the scale $a_{i}$ for each $i=1, \ldots, m$, then $\sum_{i=1}^{m} Y_{i}$ is sub-Gaussian relative to the scale $\sqrt{\sum_{i=1}^{m} a_{i}^{2}}$ (cf. Lemma 3 of [8]). Finally, if $Y$ is sub-Gaussian relative to the scale $a$, then $Y$ is a sub-1st chaos random variable relative to the scale $\sqrt{5} a$ (see Remark 3.1 of [61]).

Proof of Lemma 3.1(b). First note that $a \geq \sqrt{\operatorname{Var}\left[Y_{1}\right]}=1$. Therefore, the variable $W_{j}^{(i)}$ is sub-Gaussian relative to the scale $a$ for all $i, j$, and thus the variable $\sum_{j=1}^{N_{n}} \gamma_{n, k}(i, j) W_{j}^{(i)}$ is sub-Gaussian relative to the scale $a \sqrt{\sum_{j=1}^{N_{n}} \gamma_{n, k}(i, j)^{2}}$ for all $i=1, \ldots, N_{n}$ and $k=1, \ldots, d_{n}$. Consequently, Proposition A.2 yields

$$
E\left[\max _{1 \leq k \leq d_{n}}\left|\sum_{j=1}^{N} \gamma_{k}(i, j) W_{j}^{(i)}\right|^{3}\right] \leq\left(5 a^{2} \log \left(2 d_{n}-1+\sqrt{e}\right) \max _{1 \leq k \leq d_{n}} \sum_{j=1}^{N_{n}} \gamma_{n, k}(i, j)^{2}\right)^{3 / 2}
$$

for all $i$. This completes the proof.

\section{B.9 Proof of Corollary 3.1}

Since we have

$$
\sum_{i=1}^{N_{n}}\left(\max _{1 \leq k \leq d_{n}} \sum_{j=1}^{N_{n}} \gamma_{n, k}(i, j)^{2}\right)^{3 / 2} \leq\left(\max _{1 \leq i \leq N_{n}} \sqrt{\Lambda_{i}}\right) \sum_{i=1}^{N_{n}} \Lambda_{i}
$$

the convergence $R_{n, 1} \log ^{\frac{7}{2}} d_{n} \rightarrow 0$ follows from Lemma 3.1 and assumptions. The convergence $R_{n, 3} \log ^{3} d_{n} \rightarrow 0$ follows from Eq.(11) of [19] and assumptions.

\section{B.10 Proof of Proposition 4.1}

In the following subsections, for (possibly random) sequences $\left(x_{n}\right)$ and $\left(y_{n}\right), x_{n} \lesssim y_{n}$ means that there exists a (non-random) constant $C \in[0, \infty)$, which depends only on the model parameters such as $\sigma_{1}, \sigma_{2}$ and the constants appearing in assumptions, such that $x_{n} \leq C y_{n}$ a.s. for large $n$.

Lemma B.6. Under the assumptions of Proposition 4.1, we have

$$
\left\{1-\left(\sum_{m=1}^{M}\left|\rho_{m}\right|\right)^{2}\right\} \underline{v} \leq E\left[F_{n}(\theta)^{2}\right] \leq\left\{1+\left(\sum_{m=1}^{M}\left|\rho_{m}\right|\right)^{2}\right\} \bar{v}
$$

for every $n \in \mathbb{N}$ and every $\theta \in \mathcal{G}_{n}$.

Proof. The Isserlis formula (cf. Theorem 1.28 from [32]) yields

$$
\begin{aligned}
E\left[F_{n}(\theta)^{2}\right]= & n \sum_{I, I^{\prime} \in \Pi_{n}^{1}, J, J^{\prime} \in \Pi_{n}^{2}} \operatorname{Cov}\left[X^{1}(I) X^{2}(J), X^{1}\left(I^{\prime}\right) X^{2}\left(J^{\prime}\right)\right] K\left(I, J_{-\theta}\right) K\left(I^{\prime}, J_{-\theta}^{\prime}\right) \\
= & n \sum_{I \in \Pi_{n}^{1}, J \in \Pi_{n}^{2}} E\left[X^{1}(I)^{2}\right] E\left[X^{2}(J)^{2}\right] K\left(I, J_{-\theta}\right) \\
& +n \sum_{I, I^{\prime} \in \Pi_{n}^{1}, J, J^{\prime} \in \Pi_{n}^{2}} E\left[X^{1}(I) X^{2}\left(J^{\prime}\right)\right] E\left[X^{1}\left(I^{\prime}\right) X^{2}(J)\right] K\left(I, J_{-\theta}\right) K\left(I^{\prime}, J_{-\theta}^{\prime}\right) .
\end{aligned}
$$


Now let us set $I(S)=\bigcup_{I \in \Pi_{n}^{1}: I \cap S \neq \emptyset} I$ for every interval $S$. Then we have

$$
\begin{aligned}
& \left|n \sum_{I, I^{\prime} \in \Pi_{n}^{1}, J, J^{\prime} \in \Pi_{n}^{2}} E\left[X^{1}(I) X^{2}\left(J^{\prime}\right)\right] E\left[X^{1}\left(I^{\prime}\right) X^{2}(J)\right] K\left(I, J_{-\theta}\right) K\left(I^{\prime}, J_{-\theta}^{\prime}\right)\right| \\
& =n\left|\sum_{m, m^{\prime}} \rho_{m} \rho_{m^{\prime}} \sum_{I, J, I^{\prime}, J^{\prime}} K\left(I, J_{-\theta}\right) K\left(I^{\prime}, J_{-\theta}^{\prime}\right) \int_{I \cap J_{-\theta_{m}}^{\prime}} \sigma_{1}(t) \sigma_{2}\left(t+\theta_{m}\right) d t \int_{I^{\prime} \cap J_{-\theta_{m^{\prime}}}} \sigma_{1}(t) \sigma_{2}\left(t+\theta_{m^{\prime}}\right) d t\right| \\
& \leq n \sum_{m, m^{\prime}}\left|\rho_{m} \rho_{m^{\prime}}\right| \sum_{I, J, I^{\prime}, J^{\prime}} K\left(I, J_{-\theta}\right) K\left(I^{\prime}, J_{-\theta}^{\prime}\right) \\
& \times \sqrt{\int_{I \cap J_{-\theta_{m}}^{\prime}} \sigma_{1}(t)^{2} d t \int_{I \cap J_{-\theta_{m}}^{\prime}} \sigma_{2}\left(t+\theta_{m}\right)^{2} d t \int_{I^{\prime} \cap J_{-\theta_{m^{\prime}}}} \sigma_{1}(t)^{2} d t \int_{I^{\prime} \cap J_{-\theta_{m^{\prime}}}} \sigma_{2}\left(t+\theta_{m^{\prime}}\right)^{2} d t} \\
& \leq n \sum_{m, m^{\prime}}\left|\rho_{m^{\prime}} \rho_{m}\right| \sum_{I, J, I^{\prime}, J^{\prime}} K\left(I, J_{-\theta}\right) K\left(I^{\prime}, J_{-\theta}^{\prime}\right) \int_{I \cap J_{-\theta_{m}}^{\prime}} \sigma_{1}(t)^{2} d t \int_{I^{\prime} \cap J_{-\theta_{m^{\prime}}}} \sigma_{2}\left(t+\theta_{m^{\prime}}\right)^{2} d t \\
& =n \sum_{m, m^{\prime}}\left|\rho_{m^{\prime}} \rho_{m}\right| \sum_{I, J, J^{\prime}} K\left(I, J_{-\theta}\right) \int_{I \cap J_{-\theta_{m}}^{\prime}} \sigma_{1}(t)^{2} d t \int_{I\left(J_{-\theta}^{\prime}\right) \cap J_{-\theta_{m^{\prime}}}} \sigma_{2}\left(t+\theta_{m^{\prime}}\right)^{2} d t \\
& \leq n \sum_{m, m^{\prime}}\left|\rho_{m^{\prime}} \rho_{m}\right| \sum_{I, J} K\left(I, J_{-\theta}\right) \int_{I} \sigma_{1}(t)^{2} d t \int_{J_{-\theta_{m^{\prime}}}} \sigma_{2}\left(t+\theta_{m^{\prime}}\right)^{2} d t \\
& =n\left(\sum_{m}\left|\rho_{m}\right|\right)^{2} \sum_{I, J} K\left(I, J_{-\theta}\right) \int_{I} \sigma_{1}(t)^{2} d t \int_{J} \sigma_{2}(t)^{2} d t=\left(\sum_{m}\left|\rho_{m}\right|\right)^{2} V_{n}(\theta),
\end{aligned}
$$

hence we obtain the desired result.

Lemma B.7. Under the assumptions of Proposition 4.1, there is a constant $C>0$ such that

$$
E\left[F_{n}(\theta)^{4}\right]-3 E\left[F_{n}(\theta)^{2}\right]^{2} \leq C n r_{n}^{2}
$$

for every $n \in \mathbb{N}$ and every $\theta \in \mathcal{G}_{n}$.

Proof. First we apply the so-called reduction procedures used in $[28,29]$ to every realization of $(I)_{I \in \Pi_{n}^{1}}$ and $\left(J_{-\theta}\right)_{J \in \Pi_{n}^{2}}$ (see also the proof of Lemma 2 from [19]). We define a new partition $\tilde{\Pi}_{n}^{1}$ as follows: $I \in \tilde{\Pi}_{n}^{1}$ if and only if either $I \in \Pi_{n}^{1}$ and it has non-empty intersection with two distinct intervals from $\left\{J_{-\theta}: J \in \Pi_{n}^{2}\right\}$ or there is $J \in \Pi_{n}^{2}$ such that $I$ is the union of all intervals from $\Pi_{n}^{1}$ included in $J_{-\theta}$. We also define a new partition $\tilde{\Pi}_{n}^{2}$ as follows: $J \in \tilde{\Pi}_{n}^{2}$ if and only if either $J \in \Pi_{n}^{2}$ and $J_{-\theta}$ has non-empty intersection with two distinct intervals from $\Pi_{n}^{1}$ or there is $I \in \Pi_{n}^{1}$ such that $J$ is the union of all intervals from $J^{\prime} \in \Pi_{n}^{2}$ such that $J_{-\theta}^{\prime}$ is included in $I$. Due to bilinearity $F_{n}(\theta)$ is invariant under this procedure. $r_{N}$ is also unchanged by this application because of its definition. Moreover, by construction we have

$$
\max _{J \in \tilde{\Pi}_{n}^{2}} \sum_{I \in \tilde{\Pi}_{n}^{1}} K\left(I, J_{-\theta}\right) \leq 3, \quad \max _{I \in \tilde{\Pi}_{n}^{1}} \sum_{J \in \tilde{\Pi}_{n}^{2}} K\left(I, J_{-\theta}\right) \leq 3
$$

Consequently, for the proof we may replace $\left(\Pi_{n}^{1}, \Pi_{n}^{2}\right)$ by $\left(\tilde{\Pi}_{n}^{1}, \tilde{\Pi}_{n}^{2}\right)$. This allows us to assume that

$$
\max _{J \in \Pi_{n}^{2}} \sum_{I \in \Pi_{n}^{1}} K\left(I, J_{-\theta}\right) \leq 3, \quad \max _{I \in \Pi_{n}^{1}} \sum_{J \in \Pi_{n}^{2}} K\left(I, J_{-\theta}\right) \leq 3
$$


throughout the proof without loss of generality.

We turn to the main body of the proof. Let $\Sigma_{n}$ be the covariance matrix of $\left.\left.\left(X^{1}(I)\right)_{I \in \Pi_{n}^{1}}, X^{2}(J)\right)_{J \in \Pi_{n}^{2}}\right)^{\top}$. Set $\Gamma_{n}(\theta)=\Sigma_{n}^{1 / 2} A_{n}(\theta) \Sigma_{n}^{1 / 2}$, where

$$
A_{n}(\theta)=\left(\begin{array}{cc}
0 & K_{n}(\theta) \\
K_{n}(\theta)^{\top} & 0
\end{array}\right), \quad K_{n}(\theta)=\left(\sqrt{n} K\left(I, J_{-\theta}\right) / 2\right)_{I \in \Pi_{n}^{1}, J \in \Pi_{n}^{2}} .
$$

From Eq.(11) of [19] we have

$$
E\left[F_{n}(\theta)^{4}\right]-3 E\left[F_{n}(\theta)^{2}\right]^{2}=2^{3} 3 ! \operatorname{tr}\left(\Gamma_{n}(\theta)^{4}\right) \leq 48\left\|\Gamma_{n}(\theta)\right\|_{\mathrm{sp}}^{2}\left\|\Gamma_{n}(\theta)\right\|_{F}^{2}=24\left\|\Gamma_{n}(\theta)\right\|_{\mathrm{sp}}^{2} E\left[F_{n}(\theta)^{2}\right] .
$$

Now, Lemma B.6 yields

$$
E\left[F_{n}(\theta)^{2}\right] \leq\left\{1+\left(\sum_{m}\left|\rho_{m}\right|\right)^{2}\right\} \bar{v} .
$$

On the other hand, by Example 5.6.5 and Theorem 5.6.9 of [31] as well as (B.2), we have $\left\|A_{n}(\theta)\right\|_{\text {sp }} \leq 3 \sqrt{n} / 2$. Therefore, Corollary 4.5.11 (the Ostorowski theorem), Example 5.6.5 and Theorem 5.6.9 of [31] imply that

$$
\begin{aligned}
&\left\|\Gamma_{n}(\theta)\right\|_{\mathrm{sp}} \leq \frac{3}{2} \sqrt{n}\left\|\Sigma_{n}\right\|_{\mathrm{sp}} \leq \frac{3}{2} \sqrt{n} \max \left\{\max _{I \in \Pi_{n}^{1}} E\left[X^{1}(I)^{2}\right], \max _{J \in \Pi_{n}^{2}} E\left[X^{2}(J)^{2}\right]\right\} \\
&+\frac{3}{2} \sqrt{n} \max _{I \in \Pi_{n}^{1}} \sum_{J \in \Pi_{n}^{2}}\left|E\left[X^{1}(I) X^{2}(J)\right]\right| \\
& \lesssim \sqrt{n} r_{n}+\sqrt{n} \max _{I \in \Pi_{n}^{1}} \sum_{J \in \Pi_{n}^{2}}\left|E\left[X^{1}(I) X^{2}(J)\right]\right| .
\end{aligned}
$$

Since we have

$$
\sum_{J \in \Pi_{n}^{2}}\left|E\left[X^{1}(I) X^{2}(J)\right]\right| \leq \sum_{J \in \Pi_{n}^{2}} \sum_{m}\left|\rho_{m}\right| \int_{I \cap J_{-\theta_{m}}} \sigma_{1}(t) \sigma_{2}\left(t+\theta_{m}\right) d t \lesssim r_{n}
$$

we obtain the desired result.

Proof of Proposition 4.1. The result is an immediate consequence of Theorem 3.1 and Lemmas B.6-B.7.

\section{B.11 Proof of Proposition 4.2}

Throughout this subsection, we set

$$
\mathbb{H}=\left\{\int_{-\infty}^{\infty} f(t) d B_{t}^{1}+\int_{-\infty}^{\infty} g(t) d B_{t}^{2}: f, g \in L^{2}(\mathbb{R})\right\} .
$$

$\mathbb{H}$ is obviously a Gaussian Hilbert space. Also, for each $\nu=1,2$ we define the process $v^{\nu}=\left(v_{t}^{\nu}\right)_{t \geq 0}$ by $v_{t}^{\nu}=$ $\int_{0}^{t} \sigma_{\nu}(s)^{2} d s, t \geq 0$.

Lemma B.8. Under the assumptions of Proposition 4.2(a), we have

$$
\sup _{x \in \mathbb{R}}\left|P\left(T_{n}^{*} \leq x \mid \mathcal{F}^{X}\right)-P\left(\max _{\theta \in \mathcal{G}_{n}}\left|Z_{n}(\theta)\right| \leq x\right)\right| \rightarrow^{p} 0
$$

as $n \rightarrow \infty$, where the process $\left(Z_{n}(\theta)\right)_{\theta \in \mathcal{G}_{n}}$ is the same one as in Proposition 4.1. 
Proof. Define the $n_{1} \times n_{2}$ matrix $\Xi_{n}(\theta)$ by $\Xi_{n}(\theta)=\left(\frac{1}{2} X^{1}(I) K\left(I, J_{-\theta}\right) X^{2}(J)\right)_{I, J}$, and set

$$
\widetilde{\Xi}_{n}(\theta)=\left(\begin{array}{cc}
O & \Xi_{n}(\theta) \\
\Xi_{n}(\theta)^{\top} & O
\end{array}\right)
$$

Then we can rewrite $U_{n}^{*}(\theta)$ as $U_{n}^{*}(\theta)=\boldsymbol{w}^{\top} \widetilde{\Xi}_{n}(\theta) \boldsymbol{w}$, where $\boldsymbol{w}=\left(\left(w_{I}^{1}\right)_{I \in \Pi_{n}^{1}},\left(w_{J}^{2}\right)_{J \in \Pi_{n}^{2}}\right)^{\top}$. Therefore, by Theorem 3.2 and Lemma 3.1, it suffices to prove $R_{n, 1}^{*} \log ^{10}\left(\# \mathcal{G}_{n}\right) \vee R_{n, 2}^{*} \log ^{10}\left(\# \mathcal{G}_{n}\right) \vee R_{n, 3}^{*} \log ^{2}\left(\# \mathcal{G}_{n}\right) \vee R_{n, 4}^{*} \log ^{3}\left(\# \mathcal{G}_{n}\right) \rightarrow^{p}$ 0 , where

$$
\begin{aligned}
& R_{n, 1}^{*}=\sum_{I} \max _{\theta \in \mathcal{G}_{n}}\left(n \sum_{J} X^{1}(I)^{2} X^{2}(J)^{2} K\left(I, J_{-\theta}\right)\right)^{3 / 2}, \\
& R_{n, 2}^{*}=\sum_{J} \max _{\theta \in \mathcal{G}_{n}}\left(n \sum_{I} X^{1}(I)^{2} X^{2}(J)^{2} K\left(I, J_{-\theta}\right)\right)^{3 / 2}, \\
& R_{n, 3}^{*}=\sqrt{n} \max _{\theta, \theta^{\prime} \in \mathcal{G}_{n}}\left|E\left[U_{n}(\theta) U_{n}\left(\theta^{\prime}\right)\right]-E\left[U_{n}^{*}(\theta) U_{n}^{*}\left(\theta^{\prime}\right) \mid \mathcal{F}^{X}\right]\right|, \\
& R_{n, 4}^{*}=\max _{1 \leq k \leq d} \sqrt{E\left[Q_{n}^{*}(\theta)^{4} \mid \mathcal{F}^{X}\right]-3 E\left[Q_{n}^{*}(\theta)^{2} \mid \mathcal{F}^{X}\right]^{2}} .
\end{aligned}
$$

Here, $Q_{n}^{*}(\theta)=\sqrt{n} \boldsymbol{G}^{\top} \widetilde{\Xi}_{n}(\theta) \boldsymbol{G}$ and $\boldsymbol{G}=\left(\left(G_{I}^{1}\right)_{I \in \Pi_{n}^{1}},\left(G_{J}^{2}\right)_{J \in \Pi_{n}^{2}}\right)^{\top}$ with $\left(G_{I}^{1}\right)_{I \in \Pi_{n}^{1}}$ and $\left(G_{J}^{2}\right)_{J \in \Pi_{n}^{2}}$ being mutually independent sequence of i.i.d. standard Gaussian variables independent of $\mathcal{F}^{X}$.

First we consider $R_{n, 1}^{*}$ and $R_{n, 2}^{*}$. Noting that the inequalities

$$
\sum_{I^{\prime}} v^{1}\left(I^{\prime}\right) K\left(I^{\prime}, J_{-\theta}\right) \leq 3\left(\sup _{0 \leq t \leq T} \sigma_{1}(t)^{2}\right) r_{n}, \quad \sum_{J^{\prime}} v^{2}\left(J^{\prime}\right) K\left(I, J_{-\theta}^{\prime}\right) \leq 3\left(\sup _{0 \leq t \leq T} \sigma_{2}(t)^{2}\right) r_{n},
$$

which hold for every $n$ and all $I \in \Pi_{n}^{1}, J \in \Pi_{n}^{2}$ and $\theta \in \mathcal{G}_{n}$, by the triangular inequality we have

$$
\left\|\sum_{J} X^{2}(J)^{2} K\left(I, J_{-\theta}\right)\right\|_{2} \leq \sum_{J}\left\|X^{2}(J)^{2}\right\|_{2} K\left(I, J_{-\theta}\right) \leq \sqrt{3} \sum_{J} v^{2}(J) K\left(I, J_{-\theta}\right) \lesssim r_{n} .
$$

Since $\sum_{J} X^{2}(J)^{2} K\left(I, J_{-\theta}\right) \in \overline{\mathcal{P}}_{2}(\mathbb{H})$, the Schwarz inequality and Proposition A.2 yield

$$
\begin{aligned}
E\left[R_{n, 1}^{*}\right] & \leq n^{3 / 2} \sum_{I} \sqrt{E\left[X^{1}(I)^{6}\right]} \sqrt{E\left[\max _{\theta \in \mathcal{G}_{n}}\left(\sum_{J} X^{2}(J)^{2} K\left(I, J_{-\theta}\right)\right)^{3}\right]} \\
& \lesssim n^{3 / 2} \sqrt{r_{n}}\left\{r_{n} \log \left(\# \mathcal{G}_{n}\right)\right\}^{3 / 2} .
\end{aligned}
$$

Therefore, we obtain $R_{n, 1}^{*} \log ^{10}\left(\# \mathcal{G}_{n}\right) \rightarrow 0$ by the Markov inequality due to $r_{n}=O\left(n^{-3 / 4+\eta}\right)$. We can prove $R_{n, 2}^{*} \log ^{10}\left(\# \mathcal{G}_{n}\right) \rightarrow 0$ in a similar manner.

Next we consider $R_{n, 3}^{*}$. Since we have

$$
\begin{aligned}
& E\left[U_{n}(\theta) U_{n}\left(\theta^{\prime}\right)\right]-E\left[U_{n}^{*}(\theta) U_{n}^{*}\left(\theta^{\prime}\right) \mid \mathcal{F}^{X}\right] \\
& =\sum_{I, J}\left\{E\left[X^{1}(I)^{2} X^{2}(J)^{2}\right]-X^{1}(I)^{2} X^{2}(J)^{2}\right\} K\left(I, J_{-\theta}\right) K\left(I, J_{-\theta^{\prime}}\right) \in \overline{\mathcal{P}}_{4}(\mathbb{H})
\end{aligned}
$$

and

$$
E\left[\left|E\left[U_{n}(\theta) U_{n}\left(\theta^{\prime}\right)\right]-E\left[U_{n}^{*}(\theta) U_{n}^{*}\left(\theta^{\prime}\right) \mid \mathcal{F}^{X}\right]\right|^{2}\right]
$$




$$
\begin{aligned}
& =\sum_{I, J, I^{\prime}, J^{\prime}}\left\{E\left[X^{1}(I)^{2} X^{2}(J)^{2} X^{1}\left(I^{\prime}\right)^{2} X^{2}\left(J^{\prime}\right)^{2}\right]-E\left[X^{1}(I)^{2} X^{2}(J)^{2}\right] E\left[X^{1}\left(I^{\prime}\right)^{2} X^{2}\left(J^{\prime}\right)^{2}\right]\right\} \\
& \times K\left(I, J_{-\theta}\right) K\left(I, J_{-\theta^{\prime}}\right) K\left(I^{\prime}, J_{-\theta}^{\prime}\right) K\left(I^{\prime}, J_{-\theta^{\prime}}^{\prime}\right) \\
& \lesssim \sum_{I, J, J^{\prime}} v^{1}(I)^{2} v^{2}(J) v^{2}\left(J^{\prime}\right) K\left(I, J_{-\theta}\right) K\left(I, J_{-\theta^{\prime}}\right) K\left(I, J_{-\theta}^{\prime}\right) K\left(I, J_{-\theta^{\prime}}^{\prime}\right) \\
& +\sum_{I, J, I^{\prime}} v^{1}(I) v^{1}\left(I^{\prime}\right) v^{2}(J)^{2} K\left(I, J_{-\theta}\right) K\left(I, J_{-\theta^{\prime}}\right) K\left(I^{\prime}, J_{-\theta}\right) K\left(I^{\prime}, J_{-\theta^{\prime}}\right) \\
& \lesssim n^{-1} r_{n}^{2}
\end{aligned}
$$

by the hypothesis $H_{0}$ and (B.3), Proposition A.2 yields $E\left[R_{n, 3}^{*}\right]=O\left(\sqrt{n} r_{n} \log ^{2}\left(\# \mathcal{G}_{n}\right)\right)$. Hence we obtain $R_{n, 3}^{*} \log ^{2}\left(\# \mathcal{G}_{n}\right) \rightarrow^{p} 0$ due to the Markov inequality.

Finally we prove $R_{n, 4}^{*} \log ^{4}\left(\mathcal{G}_{n}\right) \rightarrow^{p}$ 0. From Eq.(11) of [19] we have

$$
\begin{aligned}
& E\left[Q_{n}^{*}(\theta)^{4} \mid \mathcal{F}^{X}\right]-3 E\left[Q_{n}^{*}(\theta)^{2} \mid \mathcal{F}^{X}\right]^{2}=48 n^{2} \operatorname{tr}\left(\widetilde{\Xi}_{n}(\theta)^{4}\right)=96 n^{2}\left\|\Xi_{n}(\theta) \Xi_{n}(\theta)^{\top}\right\|_{F}^{2} \\
& \lesssim n^{2} \sum_{I, I^{\prime}}\left(\sum_{J} X^{1}(I) X^{1}\left(I^{\prime}\right) X^{2}(J)^{2} K\left(I, J_{-\theta}\right) K\left(I^{\prime}, J_{-\theta}\right)\right)^{2} \\
& =n^{2} \sum_{I, I^{\prime}, J, J^{\prime}} X^{1}(I)^{2} X^{1}\left(I^{\prime}\right)^{2} X^{2}(J)^{2} X^{2}\left(J^{\prime}\right)^{2} K\left(I, J_{-\theta}\right) K\left(I^{\prime}, J_{-\theta}\right) K\left(I, J_{-\theta}^{\prime}\right) K\left(I^{\prime}, J_{-\theta}^{\prime}\right) .
\end{aligned}
$$

In particular, we obtain $E\left[Q_{n}^{*}(\theta)^{4} \mid \mathcal{F}^{X}\right]-3 E\left[Q_{n}^{*}(\theta)^{2} \mid \mathcal{F}^{X}\right]^{2} \in \overline{\mathcal{P}}_{8}(\mathbb{H})$. Moreover, the triangular and the generalized Hölder inequalities as well as (B.3) yield

$$
\begin{aligned}
& \left\|n^{2} \sum_{I, I^{\prime}, J, J^{\prime}} X^{1}(I)^{2} X^{1}\left(I^{\prime}\right)^{2} X^{2}(J)^{2} X^{2}\left(J^{\prime}\right)^{2} K\left(I, J_{-\theta}\right) K\left(I^{\prime}, J_{-\theta}\right) K\left(I, J_{-\theta}^{\prime}\right) K\left(I^{\prime}, J_{-\theta}^{\prime}\right)\right\|_{2} \\
& \lesssim n^{2} \sum_{I, I^{\prime}, J, J^{\prime}} v^{1}(I) v^{1}\left(I^{\prime}\right) v^{2}(J) v^{2}\left(J^{\prime}\right) K\left(I, J_{-\theta}\right) K\left(I^{\prime}, J_{-\theta}\right) K\left(I, J_{-\theta}^{\prime}\right) K\left(I^{\prime}, J_{-\theta}^{\prime}\right) \\
& \lesssim n^{2} r_{n}^{2} \sum_{I, J} v^{1}(I) v^{2}(J) K\left(I, J_{-\theta}\right) \leq n r_{n}^{2} \bar{v} .
\end{aligned}
$$

Therefore, Proposition A.2 implies that $E\left[R_{n, 4}^{*}\right] \lesssim \sqrt{n} r_{n} \log ^{2}\left(\# \mathcal{G}_{n}\right)$, hence the desired result follows from the Markov inequality. Thus we complete the proof.

Lemma B.9. Under the assumptions of Proposition 4.2(b), we have $T_{n} / \sqrt{n} \rightarrow^{p} \max _{1 \leq m \leq M}\left|\rho_{m}\right| \Sigma\left(\theta_{m}\right)$ as $n \rightarrow$ $\infty$.

Proof. We first note that $v_{n}=o\left(r_{n}\right)$ as $n \rightarrow \infty$. In fact, we have $T \leq \sum_{I}|I|+2 r_{n} \leq\left(n_{1}+2\right) r_{n}$ and $T \leq \sum_{J}|J|+2 r_{n} \leq\left(n_{2}+2\right) r_{n}$, hence it holds that $T-2 r_{n} \leq v_{n} \min \left\{n_{1}, n_{2}\right\} \cdot v_{n}^{-1} r_{n}$. Since $v_{n} \min \left\{n_{1}, n_{2}\right\} \rightarrow 0$ by [A4], we necessarily have $v_{n}^{-1} r_{n} \rightarrow \infty$. Therefore, without loss of generality we may assume that $v_{n} \leq r_{n}$.

First we show that

$$
\limsup _{n \rightarrow \infty} \max _{\theta \in \mathcal{G}_{n}}\left|E\left[U_{n}(\theta)\right]\right| \leq \max _{1 \leq m \leq M}\left|\rho_{m}\right| \Sigma\left(\theta_{m}\right)
$$

Noting that

$$
I \cap J_{-\theta} \neq \emptyset, I \cap J_{-\theta^{\prime}} \neq \emptyset \Rightarrow\left|\theta^{\prime}-\theta\right| \leq 2 r_{n}
$$


for any $I \in \Pi_{n}^{1}, J \in \Pi_{n}^{2}$ and $\theta, \theta^{\prime} \in \mathcal{G}_{n}$, we have

$$
\begin{aligned}
\left|E\left[U_{n}(\theta)\right]\right| & =\left|\sum_{I, J} K\left(I, J_{-\theta}\right) \sum_{m=1}^{M} \rho_{m} \int_{I \cap J_{-\theta_{m}}} \sigma_{1}(t) \sigma_{2}\left(t+\theta_{m}\right) d t\right| \\
& \leq \max _{1 \leq m \leq M}\left|\rho_{m}\right| \sum_{I, J} \int_{I \cap J_{-\theta_{m}}} \sigma_{1}(t) \sigma_{2}\left(t+\theta_{m}\right) d t
\end{aligned}
$$

for any $\theta \in \mathcal{G}_{n}$ and sufficiently large $n$. This yields (B.4).

Next we prove

$$
\liminf _{n \rightarrow \infty} \max _{\theta \in \mathcal{G}_{n}}\left|E\left[U_{n}(\theta)\right]\right| \geq \max _{1 \leq m \leq M}\left|\rho_{m}\right| \Sigma\left(\theta_{m}\right) .
$$

Let $m^{*}$ be an integer such that $\left|\rho_{m^{*}}\right| \Sigma\left(\theta_{m^{*}}\right)=\max _{1 \leq m \leq M}\left|\rho_{m}\right| \Sigma\left(\theta_{m}\right)$. By assumption [A4], for each $n \in \mathbb{N}$ there is a number $\vartheta_{n} \in \mathcal{G}_{n}$ such that $\left|\vartheta_{n}-\theta_{m^{*}}\right| \leq v_{n}$. Now noting (B.5), we have

$$
E\left[U_{n}\left(\vartheta_{n}\right)\right]=\sum_{I, J} K\left(I, J_{-\vartheta_{n}}\right) \rho_{m^{*}} \int_{I \cap J_{-\theta_{m^{*}}}} \sigma_{1}(t) \sigma_{2}\left(t+\theta_{m^{*}}\right) d t
$$

for sufficiently large $n$. Let us denote by $\ominus$ the symmetric difference between two sets. Then we have

$$
\begin{aligned}
& \left|\sum_{I, J} K\left(I, J_{-\vartheta_{n}}\right) \rho_{m^{*}}\left\{\int_{I \cap J_{-\theta_{m^{*}}}} \sigma_{1}(t) \sigma_{2}\left(t+\theta_{m^{*}}\right) d t-\int_{I \cap J_{-\vartheta_{n}}} \sigma_{1}(t) \sigma_{2}\left(t+\theta_{m^{*}}\right) d t\right\}\right| \\
& \leq \sum_{I, J} K\left(I, J_{-\vartheta_{n}}\right) \int_{J_{-\theta_{m^{*}}} \ominus J_{-\vartheta_{n}}} 1_{I}(t) \sigma_{1}(t) \sigma_{2}\left(t+\theta_{m^{*}}\right) d t \\
& \leq \sum_{J} \int_{J_{-\theta_{m^{*}}} \ominus J_{-\vartheta_{n}}} \sigma_{1}(t) \sigma_{2}\left(t+\theta_{m^{*}}\right) d t \lesssim n_{2} v_{n} .
\end{aligned}
$$

Noting that $K\left(I, J_{-\theta}\right)=K\left(I_{\theta}, J\right)$, an analogous argument yields

$$
\left|\sum_{I, J} K\left(I, J_{-\vartheta_{n}}\right) \rho_{m^{*}}\left\{\int_{I \cap J_{-\theta_{m^{*}}}} \sigma_{1}(t) \sigma_{2}\left(t+\theta_{m^{*}}\right) d t-\int_{I \cap J_{-\vartheta_{n}}} \sigma_{1}(t) \sigma_{2}\left(t+\theta_{m^{*}}\right) d t\right\}\right| \lesssim n_{1} v_{n} .
$$

Therefore, by assumption [A4] we obtain

$$
E\left[U_{n}\left(\vartheta_{n}\right)\right]=\sum_{I, J} K\left(I, J_{-\vartheta_{n}}\right) \rho_{m^{*}} \int_{I \cap J_{-\vartheta_{n}}} \sigma_{1}(t) \sigma_{2}\left(t+\theta_{m^{*}}\right) d t+o(1)=\rho_{m^{*}} \Sigma\left(\theta_{m^{*}}\right)+o(1) .
$$

Hence we conclude that

$$
\liminf _{n \rightarrow \infty} \max _{\theta \in \mathcal{G}_{n}}\left|E\left[U_{n}(\theta)\right]\right| \geq \liminf _{n \rightarrow \infty}\left|E\left[U_{n}\left(\vartheta_{n}\right)\right]\right|=\rho_{m^{*}} \Sigma\left(\theta_{m^{*}}\right)=\max _{1 \leq m \leq M}\left|\rho_{m}\right| \Sigma\left(\theta_{m}\right),
$$

which yields (B.6).

From (B.4)-(B.6) we deduce that $\max _{\theta \in \mathcal{G}_{n}}\left|E\left[U_{n}(\theta)\right]\right| \rightarrow \max _{1 \leq m \leq M}\left|\rho_{m}\right| \Sigma\left(\theta_{m}\right)$. Since we have

$$
\left|\frac{T_{n}}{\sqrt{n}}-\max _{\theta \in \mathcal{G}_{n}}\right| E\left[U_{n}(\theta)\right]|| \leq \frac{1}{\sqrt{n}} \max _{\theta \in \mathcal{G}_{n}}\left|F_{n}(\theta)\right|,
$$

the proof of the lemma is completed once we show that $\max _{\theta \in \mathcal{G}_{n}}\left|F_{n}(\theta)\right|=o_{p}(\sqrt{n})$. By Proposition A.2 and the Markov inequality, we have $\max _{\theta \in \mathcal{G}_{n}}\left|Z_{n}(\theta)\right|=O_{p}\left(\sqrt{\log \left(\# \mathcal{G}_{n}\right)}\right)$. Hence the desired result follows from Proposition 4.1. 
Lemma B.10. Under the assumptions of Proposition 4.2(b), we have $T_{n}^{*}=O_{p}\left(\log ^{2}\left(\# \mathcal{G}_{n}\right)\right)$ as $n \rightarrow \infty$.

Proof. Take $p \geq 2$ and $\theta \in \mathcal{G}_{n}$ arbitrarily. By Lemma 1 of [8] we have $E\left[\left|w_{I}^{1}\right|^{p}\right] \leq(2 b / e) \sqrt{p}$ and $E\left[\left|w_{J}^{1}\right|^{p}\right] \leq$ $(2 b / e) \sqrt{p}$ for all $I \in \Pi_{n}^{1}$ and $J \in \Pi_{n}^{2}$. Therefore, by Proposition 3.16 from [43] $w_{I}^{1}$ and $w_{J}^{2}$ are $\left(2, p,((4 b / e) p)^{-1}\right)$ hypercontractive for all $I \in \Pi_{n}^{1}$ and $J \in \Pi_{n}^{2}$ (see also the beginning of Section B.8). Therefore, by Theorem 6.5.2 from [40] it holds that

$$
E\left[\left|U_{n}^{*}(\theta)\right|^{p} \mid \mathcal{F}^{X}\right] \lesssim p^{p}\left(E\left[\left|U_{n}^{*}(\theta)\right|^{2} \mid \mathcal{F}^{X}\right]\right)^{p / 2}
$$

Since we have

$$
E\left[\left|U_{n}^{*}(\theta)\right|^{2} \mid \mathcal{F}^{X}\right]=\sum_{I \in \Pi_{n}^{1}, J \in \Pi_{n}^{2}} X^{1}(I)^{2} X^{2}(J)^{2} K\left(I, J_{-\theta}\right) \in \overline{\mathcal{P}}_{4}(\mathbb{H})
$$

and

$$
\left\|\sum_{I \in \Pi_{n}^{1}, J \in \Pi_{n}^{2}} X^{1}(I)^{2} X^{2}(J)^{2} K\left(I, J_{-\theta}\right)\right\|_{2} \lesssim \sum_{I \in \Pi_{n}^{1}, J \in \Pi_{n}^{2}} v^{1}(I) v^{2}(J) K\left(I, J_{-\theta}\right) \leq \bar{v} / n
$$

by the triangular and the Schwarz inequalities, we obtain

$$
E\left[\left(E\left[\left|U_{n}^{*}(\theta)\right|^{2} \mid \mathcal{F}^{X}\right]\right)^{p / 2}\right] \lesssim\left(p^{2} / n\right)^{p / 2}
$$

by Theorem 5.11 and Remark 5.11 from [32]. As a result, we conclude that

$$
E\left[\left|U_{n}^{*}(\theta)\right|^{p} \mid \mathcal{F}^{X}\right] \lesssim p^{2 p} / n^{p / 2}
$$

Therefore, Lemma A.7 implies that $U_{n}^{*}(\theta)$ is a sub-4th chaos random variable relative to the scale $c / \sqrt{n}$ for some constant $c>0$. Hence Proposition A.2 yields $E\left[T_{n}^{*}\right]=O\left(\log ^{2}\left(\# \mathcal{G}_{n}\right)\right)$ as $n \rightarrow \infty$. Consequently, we obtain the desired result by the Markov inequality.

Proof of Proposition 4.2. (a) We follow Step 3 in the proof of Theorem 2 from [36]. First, by Proposition 4.1, Lemma B.8 and Theorem 9.2.2 of [23] there is a sequence $\varepsilon_{n}$ of positive numbers tending to 0 such that

$$
P\left(\mathcal{E}_{n}^{c}\right) \leq \varepsilon_{n}, \quad \sup _{x \in \mathbb{R}}\left|P\left(T_{n} \leq x\right)-P\left(\sup _{\theta \in \mathcal{G}_{n}}\left|Z_{n}(\theta)\right| \leq x\right)\right| \leq \varepsilon_{n}
$$

for all $n \in \mathbb{N}$, where

$$
\mathcal{E}_{n}=\left\{\sup _{x \in \mathbb{R}}\left|P\left(\max _{\theta \in \mathcal{G}_{n}}\left|Z_{n}(\theta)\right| \leq x\right)-P\left(T_{n}^{*} \leq x \mid \mathcal{F}^{X}\right)\right| \leq \varepsilon_{n}\right\} .
$$

Next, let us denote by $q_{n}^{Z}$ the quantile function of $\max _{\theta \in \mathcal{G}_{n}}\left|Z_{n}(\theta)\right|$. Note that $\max _{\theta \in \mathcal{G}_{n}}\left|Z_{n}(\theta)\right|$ has the density because of Lemmas B.6 and A.5. Therefore, on $\mathcal{E}_{n}$ we have

$$
P\left(T_{n}^{*} \leq q_{n}^{Z}\left(1-\alpha+\varepsilon_{n}\right) \mid \mathcal{F}^{X}\right) \geq P\left(\max _{\theta \in \mathcal{G}_{n}}\left|Z_{n}(\theta)\right| \leq q_{n}^{Z}\left(1-\alpha+\varepsilon_{n}\right)\right)-\varepsilon_{n}=1-\alpha,
$$

hence on $\mathcal{E}_{n}$ it holds that $q_{n}^{*}(1-\alpha) \leq q_{n}^{Z}\left(1-\alpha+\varepsilon_{n}\right)$. Therefore, we obtain

$$
P\left(T_{n}<q_{n}^{*}(1-\alpha)\right) \leq P\left(T_{n}<q_{n}^{Z}\left(1-\alpha+\varepsilon_{n}\right)\right)+P\left(\mathcal{E}_{n}^{c}\right)
$$




$$
\leq P\left(\max _{\theta \in \mathcal{G}_{n}}\left|Z_{n}(\theta)\right|<q_{n}^{Z}\left(1-\alpha+\varepsilon_{n}\right)\right)+2 \varepsilon_{n}=1-\alpha+3 \varepsilon_{n} .
$$

On the other hand, for any $\omega \in \mathcal{E}_{n}$ and any $z \in \mathbb{R}$ such that $P\left(T_{n}^{*} \leq z \mid \mathcal{F}^{X}\right)(\omega) \geq 1-\alpha$, we have

$$
P\left(\max _{\theta \in \mathcal{G}_{n}}\left|Z_{n}(\theta)\right| \leq q_{n}^{Z}\left(1-\alpha-\varepsilon_{n}\right)\right)=1-\alpha-\varepsilon_{n} \leq P\left(T_{n}^{*} \leq z \mid \mathcal{F}^{X}\right)(\omega)-\varepsilon_{n} \leq P\left(\max _{\theta \in \mathcal{G}_{n}}\left|Z_{n}(\theta)\right| \leq z\right),
$$

hence it holds that $q_{n}^{Z}\left(1-\alpha-\varepsilon_{n}\right) \leq z$. This implies that $q_{n}^{*}(1-\alpha) \geq q_{n}^{Z}\left(1-\alpha-\varepsilon_{n}\right)$ on $\mathcal{E}_{n}$. Therefore, we obtain

$$
\begin{aligned}
P\left(T_{n}<q_{n}^{*}(1-\alpha)\right) & \geq P\left(T_{n}<q_{n}^{Z}\left(1-\alpha-\varepsilon_{n}\right)\right)-P\left(\mathcal{E}_{n}^{c}\right) \\
& \geq P\left(\max _{\theta \in \mathcal{G}_{n}}\left|Z_{n}(\theta)\right|<q_{n}^{Z}\left(1-\alpha-\varepsilon_{n}\right)\right)-2 \varepsilon_{n}=1-\alpha-3 \varepsilon_{n} .
\end{aligned}
$$

Consequently, we obtain $P\left(T_{n}<q_{n}^{*}(1-\alpha)\right) \rightarrow 1-\alpha$ as $n \rightarrow \infty$.

(b) By the definition of $q_{n}^{*}(1-\alpha)$ we have

$$
P\left(T_{n}<q_{n}^{*}(1-\alpha)\right) \leq P\left(P\left(T_{n}^{*} \leq T_{n} \mid \mathcal{F}^{X}\right)<1-\alpha\right) \leq \alpha^{-1} P\left(T_{n}^{*}>T_{n}\right) .
$$

Since Lemmas B.9-B.10 yield $\left(T_{n}^{*}-T_{n}\right) / \sqrt{n} \rightarrow^{p}-\max _{1 \leq m \leq M}\left|\rho_{m}\right| \Sigma\left(\theta_{m}\right)$, we have

$$
\limsup _{n \rightarrow \infty} P\left(T_{n}^{*}>T_{n}\right) \leq P\left(-\max _{1 \leq m \leq M}\left|\rho_{m}\right| \Sigma\left(\theta_{m}\right) \geq 0\right)=0
$$

under $H_{1}$, hence we obtain $P\left(T_{n}<q_{n}^{*}(1-\alpha)\right) \rightarrow 0$. This implies the desired result.

\section{B.12 Proof of Proposition 4.3}

We begin by proving some auxiliary results.

Lemma B.11. Suppose that the function $\Psi: \mathbb{R} \rightarrow \mathbb{R}$ is Lipschitz continuous and compactly supported. Under the assumptions of Proposition 4.3, we have

$$
\sup _{t \in\left[a_{n}, T-a_{n}\right]}\left|\frac{1}{n} \sum_{i=1}^{n} \Psi_{h}\left(t_{i-1}-t\right)-\int_{-\infty}^{\infty} \Psi(s) d s\right|=O\left((n h)^{-1}\right)
$$

as $n \rightarrow \infty$.

Proof. Noting that $\int_{-\infty}^{\infty} \Psi(s) d s=\int_{-\infty}^{\infty} \Psi_{h}(s-t) d s$, we can decompose the target quantity as

$$
\begin{aligned}
& \sup _{t \in\left[a_{n}, T-a_{n}\right]}\left|\frac{1}{n} \sum_{i=1}^{n} \Psi_{h}\left(t_{i-1}-t\right)-\int_{-\infty}^{\infty} \Psi_{h}(s) d s\right| \\
& \leq \sup _{t \in\left[a_{n}, T-a_{n}\right]}\left\{\sum_{i=1}^{n} \int_{t_{i-1}}^{t_{i}}\left|\Psi_{h}\left(t_{i-1}-t\right)-\Psi_{h}(s-t)\right| d s+\int_{-\infty}^{0}\left|\Psi_{h}(s-t)\right| d s+\int_{T}^{\infty}\left|\Psi_{h}(s-t)\right| d s\right\} \\
& =: \mathbb{I}_{n}+\mathbb{I} \mathbb{I}_{n}+\mathbb{I} \mathbb{I}_{n} .
\end{aligned}
$$

Since $\Psi$ is Lipschitz continuous and compactly supported, we have $\mathbb{I}_{n}=O\left((n h)^{-1}\right)$ as $n \rightarrow \infty$. Moreover, since $a_{n} / h \rightarrow \infty$ as $n \rightarrow \infty, \mathbb{I I}_{n}=\mathbb{I I I} \mathbb{I}_{n}=0$ for sufficiently large $n$. This completes the proof.

Lemma B.12. Under the assumptions of Proposition 4.3, there are constants $C_{1}, C_{2}>0$ such that

$$
\frac{C_{1}}{\sqrt{n h}} \leq \mathfrak{s}_{n}(t) \leq \frac{C_{2}}{\sqrt{n h}}
$$

for any $n \in \mathbb{N}$ and $t \in\left[a_{n}, T-a_{n}\right]$. 
Proof. Lemma B.11 yields

$$
n h \cdot \mathfrak{s}_{n}^{2}(t)=\frac{2}{n h} \sum_{i=1}^{n} K\left(\frac{t_{i-1}-t}{h}\right)^{2}=2 \int_{-\infty}^{\infty} K(s)^{2} d s+O\left((n h)^{-1}\right)
$$

uniformly in $t \in\left[a_{n}, T-a_{n}\right]$ as $n \rightarrow \infty$. Since $0<\int_{-\infty}^{\infty} K(s)^{2} d s<\infty$, we obtain the desired result.

Now we turn to the main body of the proof. Let us set

$$
b_{n}(t)=\sum_{i=1}^{n} K_{h}\left(t_{i-1}-t\right) \int_{t_{i-1}}^{t_{i}} \sigma^{2}(s) d s-\sigma^{2}(t)
$$

and

$$
M_{n}(t)=2 \sum_{i=1}^{n} K_{h}\left(t_{i-1}-t\right) \int_{t_{i-1}}^{t_{i}} \int_{t_{i-1}}^{s} \sigma(r) d B_{r} \sigma(s) d B_{s}
$$

for $t \in[0, T]$. By Itô's formula we have

$$
\widehat{\sigma}_{n}^{2}(t)-\sigma^{2}(t)=M_{n}(t)+b_{n}(t) .
$$

The term $b_{n}(t)$ behaves as a bias and it is negligible because we consider an undersmoothing situation such that $n h^{1+2 \gamma} \log n \rightarrow 0$. More precisely, we have the following:

Lemma B.13. Suppose that [SB1] and [B2] are satisfied. Then there is a constant $C>0$ such that

$$
\sup _{t \in\left[a_{n}, T-a_{n}\right]}\left|b_{n}(t)\right| \leq C\left\{(n h)^{-1}+h^{\gamma}\right\}
$$

for sufficiently large $n$.

Proof. We decompose $b_{n}(t)$ as

$$
\begin{aligned}
b_{n}(t) & =\sum_{i=1}^{n} K_{h}\left(t_{i-1}-t\right) \int_{t_{i-1}}^{t_{i}}\left\{\sigma^{2}(s)-\sigma^{2}(t)\right\} d s+\sigma^{2}(t)\left\{\frac{1}{n} \sum_{i=1}^{n} K_{h}\left(t_{i-1}-t\right)-\int_{-\infty}^{\infty} K_{h}(s-t) d s\right\} \\
& =: \mathbb{I}_{n}(t)+\mathbb{I I}_{n}(t) .
\end{aligned}
$$

By assumptions we have $\sup _{t \in\left[a_{n}, T-a_{n}\right]}\left|\mathbb{I}_{n}(t)\right| \lesssim h^{\gamma}$ for sufficiently large $n$. In the meantime, Lemma B.11 and [SB1] yield $\sup _{t \in\left[a_{n}, T-a_{n}\right]}\left|\mathbb{I} \mathbb{I}_{n}(t)\right| \lesssim(n h)^{-1}$. This completes the proof.

Meanwhile, the term $M_{n}(t)$ is a martingale part and the source of stochastic estimation errors. To apply our theory to the investigation of this term, we approximate $M_{n}(t) / \sigma^{2}(t) \mathfrak{s}_{n}(t)$ by a double Wiener-Itô integral. More precisely, we define

$$
M_{n}^{0}(t)=2 \sum_{i=1}^{n} K_{h}\left(t_{i-1}-t\right) \int_{t_{i-1}}^{t_{i}} \int_{t_{i-1}}^{s} d B_{r} d B_{s}, \quad t \in[0, T] .
$$

Then we show that $M_{n}(t) /\left\{\sigma^{2}(t) \mathfrak{s}_{n}(t)\right\}$ is well-approximated by

$$
F_{n}(t)=M_{n}^{0}(t) / \mathfrak{s}_{n}(t)
$$

uniformly in $t \in[0, T]$. The proof of this approximation is motivated by the argument from Appendix A of Sabel [57] and relies on the following Burkholder-Davis-Gundy inequality with a sharp constant due to Barlow and Yor [4]: 
Proposition B.1 (Barlow and Yor [4], Proposition 4.2). There is a universal constant $c>0$ such that

$$
\left\|\sup _{0 \leq t \leq T}\left|M_{t}\right|\right\|_{p} \leq c \sqrt{p}\left\|\langle M\rangle_{T}^{1 / 2}\right\|_{p}
$$

for any $p \geq 2$ and any continuous martingale $M=\left(M_{t}\right)_{t \in[0, T]}$ with $M_{0}=0$.

To simplify notation, for a random variable $Y$, we denote its Orlictz norm based on the function $\psi_{1}(x)=e^{x}-1$ by $\|Y\|_{\psi_{1}}$ (cf. page 95 of [59]):

$$
\|Y\|_{\psi_{1}}=\inf \left\{C>0: \psi_{1}(|Y| / C) \leq 1\right\} .
$$

Note that $\|Y\|_{\psi_{1}} \leq M$ if $Y$ is a sub-2nd chaos random variable relative to the scale $M$. Conversely, $Y$ is a sub-2nd chaos random variable relative to the scale $M$ if $\|Y\|_{\psi_{1}}<M$.

Lemma B.14. Suppose that [SB1] and [B2] are satisfied. Then

$$
\left\|\sup _{t \in[0, T]}\left|\frac{M_{n}(t)}{\sigma^{2}(t) \mathfrak{s}_{n}(t)}-F_{n}(t)\right|\right\|_{\psi_{1}}=O\left(h^{\gamma}(\log n)^{2}\right)
$$

as $n \rightarrow \infty$.

Proof. Since $K$ is compactly supported, there is a constant $\ell>0$ such that the support of $K$ is contained in $[-\ell, \ell]$. Then we decompose the target quantity as

$$
\begin{aligned}
& \frac{M_{n}(t)}{\sigma^{2}(t) \mathfrak{s}_{n}(t)}-F_{n}(t) \\
& =\frac{M_{n}(t)-\sigma^{2}\left((t-\ell h)_{+}\right) M_{n}^{0}(t)}{\sigma^{2}(t) \mathfrak{s}_{n}(t)}+\frac{\left\{\sigma^{2}\left((t-\ell h)_{+}\right)-\sigma^{2}(t)\right\} M_{n}^{0}(t)}{\sigma^{2}(t) \mathfrak{s}_{n}(t)} \\
& =: \mathbf{I}_{n}(t)+\mathbf{I I}_{n}(t),
\end{aligned}
$$

where $(t-\ell h)_{+}=(t-\ell h) \vee 0$.

First we consider $\mathbf{I}_{n}(t)$. By [SB1] and Lemma B.12, it is enough to prove

$$
\left\|\sup _{t \in[0, T]} \mid \sqrt{n h}\left\{M_{n}(t)-\sigma^{2}\left((t-\ell h)_{+}\right) M_{n}^{0}(t)\right\}\right\| \| h^{\gamma}(\log n)^{2}
$$

for all $n \in \mathbb{N}$. We decompose $\sqrt{n h}\left\{M_{n}(t)-\sigma^{2}\left((t-\ell h)_{+}\right) M_{n}^{0}(t)\right\}$ as

$$
\begin{aligned}
& \sqrt{n h}\left\{M_{n}(t)-\sigma^{2}\left((t-\ell h)_{+}\right) M_{n}^{0}(t)\right\} \\
& =2 \sqrt{n h} \sum_{i=1}^{n} K_{h}\left(t_{i-1}-t\right) \int_{t_{i-1}}^{t_{i}} \int_{t_{i-1}}^{s}\left\{\sigma(r)-\sigma\left((t-\ell h)_{+}\right)\right\} d B_{r} \sigma(s) d B_{s} \\
& \quad+2 \sqrt{n h} \sum_{i=1}^{n} K_{h}\left(t_{i-1}-t\right) \int_{t_{i-1}}^{t_{i}} \int_{t_{i-1}}^{s} \sigma\left((t-\ell h)_{+}\right) d B_{r}\left\{\sigma(s)-\sigma\left((t-\ell h)_{+}\right) d B_{s}\right. \\
& =: \mathbf{A}_{n}(t)+\mathbf{B}_{n}(t) .
\end{aligned}
$$

Let us consider $\mathbf{A}_{n}(t)$. Set $u_{j}=(j h) \wedge T$ for $j=0,1, \ldots$ We obviously have

$$
\sup _{t \in[0, T]}\left|\mathbf{A}_{n}(t)\right| \leq \max _{j=0,1, \ldots,\lfloor T / h\rfloor}\left(\left|\mathbf{A}_{n}\left(u_{j}\right)\right|+\sup _{t \in\left[u_{j}, u_{j+1}\right]}\left|\mathbf{A}_{n}(t)-\mathbf{A}_{n}\left(u_{j}\right)\right|\right) .
$$


To estimate the second term on the right side, we first show that there is a constant $a_{0}>0$ such that, for every $j=0,1, \ldots,\lfloor T / h\rfloor$, the process $\mathbf{A}_{n}^{j}(t):=\mathbf{A}_{n}(t)-\mathbf{A}_{n}\left(u_{j}\right)$ indexed by $t \in\left[u_{j}, u_{j+1}\right]$ is a sub-2nd-Gaussian chaos process with respect to the semi-metric $\mathfrak{d}^{j}$ defined by

$$
\mathfrak{d}^{j}\left(t, t^{\prime}\right)=a_{0}\left|t-t^{\prime}\right|^{\gamma}, \quad t, t^{\prime} \in\left[u_{j}, u_{j+1}\right]
$$

To prove this, fix a $j \in\{0,1, \ldots,\lfloor T / h\rfloor\}$ and take $t, t^{\prime} \in\left[u_{j}, u_{j+1}\right]$ arbitrarily. We may assume $t \leq t^{\prime}$ without loss of generality. Then, we can decompose $\mathbf{A}_{n}^{j}(t)-\mathbf{A}_{n}^{j}\left(t^{\prime}\right)$ as

$$
\begin{aligned}
& \mathbf{A}_{n}^{j}(t)-\mathbf{A}_{n}^{j}\left(t^{\prime}\right) \\
& =\int_{0}^{T} \int_{0}^{s} \sum_{i=1}^{n} \Psi_{n, i}\left(t, t^{\prime}\right) 1_{\left(t_{i-1}, t_{i}\right]}(s) 1_{\left(t_{i-1}, s\right]}(r)\left\{\sigma(r)-\sigma\left((t-\ell h)_{+}\right)\right\} d B_{r} \sigma(s) d B_{s} \\
& \quad+\int_{0}^{T} \int_{0}^{s} \sum_{i=1}^{n} 2 \sqrt{n h} K_{h}\left(t_{i-1}-t^{\prime}\right) 1_{\left(t_{i-1}, t_{i}\right]}(s) 1_{\left(t_{i-1}, s\right]}(r)\left\{\sigma\left(\left(t^{\prime}-\ell h\right)_{+}\right)-\sigma\left((t-\ell h)_{+}\right)\right\} d B_{r} \sigma(s) d B_{s} \\
& =: \Delta_{n, 1}^{j}\left(t, t^{\prime}\right)+\Delta_{n, 2}^{j}\left(t, t^{\prime}\right),
\end{aligned}
$$

where $\Psi_{n, i}\left(t, t^{\prime}\right)=2 \sqrt{n h}\left\{K_{h}\left(t_{i-1}-t\right)-K_{h}\left(t_{i-1}-t^{\prime}\right)\right\}$. Regarding $\Delta_{n, 1}^{j}\left(t, t^{\prime}\right)$, for every $p>1$, Proposition B.1, the Lyapunov inequality, [SB1] and [B2] yield

$$
\begin{aligned}
& \left\|\Delta_{n, 1}^{j}\left(t, t^{\prime}\right)\right\|_{p} \\
& \lesssim \sqrt{p}\left\|\left\{\int_{0}^{T}\left(\int_{0}^{s} \sum_{i=1}^{n} \Psi_{n, i}\left(t, t^{\prime}\right) 1_{\left(t_{i-1}, t_{i}\right]}(s) 1_{\left(t_{i-1}, s\right]}(r)\left\{\sigma(r)-\sigma\left(\left(u_{j}-\ell h\right)_{+}\right)\right\} d B_{r}\right)^{2} \sigma^{2}(s) d s\right\}^{1 / 2}\right\|_{p} \\
& \lesssim \sqrt{p}\left\|\int_{0}^{T}\left(\int_{0}^{s} \sum_{i=1}^{n} \Psi_{n, i}\left(t, t^{\prime}\right) 1_{\left(t_{i-1}, t_{i}\right]}(s) 1_{\left(t_{i-1}, s\right]}(r)\left\{\sigma(r)-\sigma\left(\left(u_{j}-\ell h\right)_{+}\right)\right\} d B_{r}\right)^{2} d s\right\|_{p}^{1 / 2} \\
& \leq \sqrt{p} T^{\frac{1-p^{-1}}{2}}\left(\int_{0}^{T}\left\|\int_{0}^{s} \sum_{i=1}^{n} \Psi_{n, i}\left(t, t^{\prime}\right) 1_{\left(t_{i-1}, t_{i}\right]}(s) 1_{\left(t_{i-1}, s\right]}(r)\left\{\sigma(r)-\sigma\left(\left(u_{j}-\ell h\right)_{+}\right)\right\} d B_{r}\right\|_{2 p}^{2} d s\right)^{1 / 2} \\
& \lesssim p\left(\int_{0}^{T}\left\|\left\{\int_{0}^{s} \sum_{i=1}^{n} \Psi_{n, i}\left(t, t^{\prime}\right)^{2} 1_{\left(t_{i-1}, t_{i}\right]}(s) 1_{\left(t_{i-1}, s\right]}(r)\left\{\sigma(r)-\sigma\left(\left(u_{j}-\ell h\right)_{+}\right)\right\}^{2} d r\right\}^{1 / 2}\right\|_{2 p}^{2} d s\right)^{1 / 2} \\
& \lesssim p h^{\gamma}\left(\frac{1}{n^{2}} \sum_{i=1}^{n} \Psi_{n, i}\left(t, t^{\prime}\right)^{2}\right)^{1 / 2} \lesssim p \frac{\left|t-t^{\prime}\right|}{h^{1-\gamma}} \leq p\left|t-t^{\prime}\right|^{\gamma},
\end{aligned}
$$

where we use the inequality $\left|t-t^{\prime}\right| \leq h$ which holds because $t, t^{\prime} \in\left[u_{j}, u_{j+1}\right]$. Analogously we have

$$
\left\|\Delta_{n, 2}^{j}\left(t, t^{\prime}\right)\right\|_{p} \lesssim p\left|t-t^{\prime}\right|^{\gamma}
$$

Therefore, by Lemma A.7 we obtain the desired result. Now, since we have

$$
\int_{0}^{a_{0} h^{\gamma}} \log N\left(\left[u_{j}, u_{j+1}\right], \mathfrak{d}_{0}, r\right) d r \lesssim \int_{0}^{a_{0} h^{\gamma}} \log \frac{1}{r^{1 / \gamma}} d r \lesssim h^{\gamma} \log n
$$


by Proposition A.3 it holds that $\left\|\sup _{t \in\left[u_{j}, u_{j+1}\right]}\left|\mathbf{A}_{n}^{j}(t)\right|\right\|_{\psi_{1}} \lesssim h^{\gamma} \log n$ for every $j=0,1, \ldots,\lfloor T / h\rfloor$. Thus, Lemma 2.2.2 of [59] implies that

$$
\left\|\max _{j=0,1, \ldots,\lfloor T / h\rfloor} \sup _{t \in\left[u_{j}, u_{j+1}\right]}\left|\mathbf{A}_{n}^{j}(t)\right|\right\|_{\psi_{1}} \lesssim h^{\gamma}(\log n)^{2}
$$

for all $n \in \mathbb{N}$. In the meantime, an analogous (simpler) argument to the above implies that $\left\|\mathbf{A}_{n}\left(u_{j}\right)\right\|_{\psi_{1}} \lesssim h^{\gamma}$ for every $j=0,1, \ldots,\lfloor T / h\rfloor$. Therefore, Lemma 2.2.2 of [59] again implies that

$$
\left\|\max _{j=0,1, \ldots,\lfloor T / h\rfloor}\left|\mathbf{A}_{n}\left(u_{j}\right)\right|\right\|_{\psi_{1}} \lesssim h^{\gamma} \log n
$$

for all $n \in \mathbb{N}$. Overall, we conclude that

$$
\left\|\sup _{t \in[0, T]}\left|\mathbf{A}_{n}(t)\right|\right\|_{\psi_{1}} \lesssim h^{\gamma}(\log n)^{2}
$$

for all $n \in \mathbb{N}$.

Similarly, we can prove $\left\|\sup _{t \in[0, T]}\left|\mathbf{B}_{n}(t)\right|\right\|_{\psi_{1}} \lesssim h^{\gamma} \log n$. This completes the proof of (B.7).

Next we consider $\mathbf{I I}_{n}(t)$. [SB1] and Lemma B.12 yield

$$
\left\|\sup _{t \in[0, T]}\left|\mathbf{I I}_{n}(t)\right|\right\|_{\psi_{1}} \lesssim h^{\gamma}\left\|\sup _{t \in[0, T]}\left|\sqrt{n h} M_{n}^{0}(t)\right|\right\|_{\psi_{1}} .
$$

Now, by Proposition A.1 $\left(\sqrt{n h} M_{n}^{0}(t)\right)_{t \in[0, T]}$ is a sub-2nd-Gaussian chaos process with respect to the semi-metric $\mathfrak{d}_{0}$ defined by

$$
\mathfrak{d}_{0}\left(t, t^{\prime}\right)=a_{3} \sqrt{\frac{1}{n^{2}} \sum_{i=1}^{n}\left\{K_{h}\left(t_{i-1}-t\right)-K_{h}\left(t_{i-1}-t^{\prime}\right)\right\}^{2}}, \quad t, t^{\prime} \in[0, T]
$$

for some constant $a_{3}>0$, hence Proposition A.3 yields

$$
\begin{aligned}
\left\|\sup _{t \in[0, T]}\left|\sqrt{n h} M_{n}^{0}(t)\right|\right\|_{\psi_{1}} & \lesssim\left\|\sqrt{n h} M_{n}^{0}(0)\right\|_{\psi_{1}}+\int_{0}^{2} \log N\left([0, T], \mathfrak{d}_{0}, r\right) d r \\
& \lesssim 1+\int_{0}^{2} \log \left((r h)^{-1}\right) d r \lesssim \log n .
\end{aligned}
$$

This completes the proof.

Thanks to the above lemma, we can work with the process $F_{n}(t)$ to prove the Gaussian approximation result stated in Proposition 4.3. Note that we have

$$
\operatorname{Var}\left[M_{n}^{0}(t)\right]=\mathfrak{s}_{n}^{2}(t)
$$

for every $t$, hence $E\left[F_{n}(t)^{2}\right]=1$ for every $t$.

Lemma B.15. Under the assumptions of Proposition 4.3, there is a constant $C>0$ such that

$$
E\left[\left|F_{n}(s)-F_{n}(t)\right|^{2}\right] \leq C \frac{(s-t)^{2}}{h^{2}}
$$

for any $n \in \mathbb{N}$ and $s, t \in[0, T]$. 
Proof. For any $s, t \in[0, T]$ we have

$$
\begin{aligned}
& E\left[\left|F_{n}(s)-F_{n}(t)\right|^{2}\right]=\operatorname{Var}\left[\frac{M_{n}^{0}(s)}{\mathfrak{s}_{n}(s)}-\frac{M_{n}^{0}(t)}{\mathfrak{s}_{n}(t)}\right]=\frac{\operatorname{Var}\left[M_{n}^{0}(s) \mathfrak{s}_{n}(t)-M_{n}^{0}(t) \mathfrak{s}_{n}(s)\right]}{\mathfrak{s}_{n}^{2}(s) \mathfrak{s}_{n}^{2}(t)} \\
& \leq 2 \frac{\operatorname{Var}\left[M_{n}^{0}(s)\right]\left(\mathfrak{s}_{n}(t)-\mathfrak{s}_{n}(s)\right)^{2}+\mathfrak{s}_{n}(s)^{2} \operatorname{Var}\left[M_{n}^{0}(s)-M_{n}^{0}(t)\right]}{\mathfrak{s}_{n}(s)^{2} \mathfrak{s}_{n}(t)^{2}} \\
& =2 \frac{\left(\mathfrak{s}_{n}(t)-\mathfrak{s}_{n}(s)\right)^{2}+\operatorname{Var}\left[M_{n}^{0}(s)-M_{n}^{0}(t)\right]}{\mathfrak{s}_{n}(t)^{2}} \leq 4 \frac{\operatorname{Var}\left[M_{n}^{0}(s)-M_{n}^{0}(t)\right]}{\mathfrak{s}_{n}(t)^{2}},
\end{aligned}
$$

where we use the inequality $\left|\sqrt{\operatorname{Var}\left[Y_{1}\right]}-\sqrt{\operatorname{Var}\left[Y_{2}\right]}\right|^{2} \leq \operatorname{Var}\left[Y_{1}-Y_{2}\right]$ holding for any random variables $Y_{1}, Y_{2}$. Now, since $K$ is Lipschitz continuous and compactly supported, it holds that

$$
\begin{aligned}
\operatorname{Var}\left[M_{n}^{0}(s)-M_{n}^{0}(t)\right] & =2 \sum_{i=1}^{n}\left|K_{h}\left(t_{i-1}-s\right)-K_{h}\left(t_{i-1}-t\right)\right|^{2} \frac{1}{n^{2}} \\
& \lesssim n h \cdot \frac{(s-t)^{2}}{h^{4}} \cdot \frac{1}{n^{2}}=\frac{(s-t)^{2}}{n h^{3}} .
\end{aligned}
$$

Now the desired result follows from Lemma B.12.

Lemma B.16. Under the assumptions of Proposition 4.3, it holds that

$$
E\left[\sup _{t \in[0, T]}\left|Z_{n}(t)\right|\right]=O(\sqrt{\log n}), \quad\left\|w\left(Z_{n} ; n^{-1}\right)\right\|_{\psi_{1}}=O\left(\frac{\sqrt{\log n}}{n h}\right)
$$

as $n \rightarrow \infty$. Moreover, there is a constant $C_{0}>0$ such that

$$
\sup _{x \in \mathbb{R}} P\left(\left|\sup _{t \in\left[a_{n}, T-a_{n}\right]}\right| Z_{n}(t)|-x| \leq \varepsilon\right) \leq C_{0} \varepsilon \sqrt{\log n}
$$

for any $\varepsilon>0$ and any $n \in \mathbb{N}$.

Proof. Define the semi-metric $\mathfrak{d}_{n}$ on $[0, T]$ by $\mathfrak{d}_{n}(s, t)=\sqrt{E\left[\left|Z_{n}(s)-Z_{n}(t)\right|^{2}\right]}, s, t \in[0, T]$. Note that we have $\mathfrak{d}_{n}(s, t) \leq 2$ and $\mathfrak{d}_{n}(s, t)=\sqrt{E\left[\left|F_{n}(s)-F_{n}(t)\right|^{2}\right]}$ for all $s, t \in[0, T]$ by the definition of $Z_{n}$. Therefore, the Dudley maximal inequality (cf. Corollary 2.2 .8 of [59]) and Lemma B.15 imply that

$$
E\left[\sup _{t \in[0, T]}\left|Z_{n}(t)\right|\right] \lesssim 1+\int_{0}^{2} \sqrt{\log \left(N\left([0, T], \mathfrak{d}_{n}, \eta\right)\right)} d \eta \lesssim 1+\int_{0}^{2} \sqrt{\log (\eta h)^{-1}} d \eta \lesssim \sqrt{\log n}
$$

Moreover, since the process $Z_{n}(t) / \sqrt{3}$ is a sub-1st-Gaussian chaos process with respect $\mathfrak{d}_{n}$, Proposition A.3 yields

$$
\left\|w\left(Z_{n} ; n^{-1}\right)\right\|_{\psi_{1}} \lesssim \int_{0}^{\sqrt{C} / n h} \sqrt{\log \left(N\left([0, T], \mathfrak{d}_{n}, \eta\right)\right)} d \eta \lesssim \int_{0}^{\sqrt{C} / n h} \sqrt{\log (\eta h)^{-1}} d \eta \lesssim \frac{\sqrt{\log n}}{n h}
$$

where $C$ denotes the constant appearing in Lemma B.15. Hence we obtain (B.8).

Next, Lemma A.6 yields

$$
\sup _{x \in \mathbb{R}} P\left(\left|\sup _{t \in\left[a_{n}, T-a_{n}\right]}\right| Z_{n}(t)|-x| \leq \varepsilon\right) \leq 4 \varepsilon\left(E\left[\sup _{t \in\left[a_{n}, T-a_{n}\right]}\left|Z_{n}(t)\right|\right]+1\right)
$$

for any $\varepsilon>0$. Hence the latter claim follows from (B.8). 
Let us set

$$
\begin{aligned}
z_{n}(t) & =\frac{\widehat{\sigma}_{n}^{2}(t)-\sigma^{2}(t)}{\sigma^{2}(t) \mathfrak{s}_{n}(t)}, \quad t \in[0, T], \\
e_{n} & =\sup _{t \in\left[a_{n}, T-a_{n}\right]}\left|\frac{M_{n}(t)}{\sigma^{2}(t) \mathfrak{s}_{n}(t)}-F_{n}(t)\right|, \\
v_{n} & =\sqrt{n h^{1+2 \gamma}}+(n h)^{-1 / 2} .
\end{aligned}
$$

Lemma B.17. Suppose that [SB1] and [B2] are satisfied. Then there are constants $c_{1}, c_{2}>0$ such that

$$
\begin{aligned}
P\left(\sup _{t \in\left[a_{n}, T-a_{n}\right]}\left|z_{n}(t)\right| \in A\right) \leq P\left(\sup _{t \in\left[a_{n}, T-a_{n}\right]}\left|Z_{n}(t)\right| \in A^{c_{1} v_{n}+8 \varepsilon}\right) & +\varepsilon^{-2} \frac{c_{2}}{\sqrt{n h}} \log ^{2} n \\
& +P\left(e_{n}>\varepsilon\right)+P\left(w\left(F_{n} ; n^{-1}\right)>\varepsilon\right)+P\left(w_{n}\left(Z_{n} ; n^{-1}\right)>\varepsilon\right)
\end{aligned}
$$

for any $\varepsilon>0$, any Borel set $A$ of $\mathbb{R}$ and any $n \in \mathbb{N}$.

Proof. First, by Lemmas B.12-B.13 there is a constant $c_{1}>0$ such that $\sup _{t \in\left[a_{n}, T-a_{n}\right]}\left|b_{n}(t) / \mathfrak{s}_{n}(t)\right| \leq c_{1} v_{n}$. Then we have

$$
P\left(\sup _{t \in\left[a_{n}, T-a_{n}\right]}\left|z_{n}(t)\right| \in A\right) \leq P\left(\sup _{t \in\left[a_{n}, T-a_{n}\right]}\left|\frac{M_{n}(t)}{\sigma^{2}(t) \mathfrak{s}_{n}(t)}\right| \in A^{c_{1} v_{n}}\right) .
$$

Moreover, by definition we also have

$$
P\left(\sup _{t \in\left[a_{n}, T-a_{n}\right]}\left|\frac{M_{n}(t)}{\sigma^{2}(t) \mathfrak{s}_{n}(t)}\right| \in A^{c_{1} v_{n}}\right) \leq P\left(\sup _{t \in\left[a_{n}, T-a_{n}\right]}\left|F_{n}(t)\right| \in A^{c_{1} v_{n}+\varepsilon}\right)+P\left(e_{n}>\varepsilon\right) .
$$

Next, let us set $s_{j}^{n}=a_{n}+j / n$ for $j=1, \ldots, N:=\left\lfloor\left(T-2 a_{n}\right) n\right\rfloor$. By definition it holds that

$$
\begin{aligned}
& \max _{1 \leq j \leq N}\left|F_{n}\left(s_{j}^{n}\right)\right| \leq \sup _{t \in\left[a_{n}, T-a_{n}\right]}\left|F_{n}(t)\right| \leq \max _{1 \leq j \leq N}\left|F_{n}\left(s_{j}^{n}\right)\right|+w\left(F_{n} ; n^{-1}\right), \\
& \max _{1 \leq j \leq N}\left|Z_{n}\left(s_{j}^{n}\right)\right| \leq \sup _{t \in\left[a_{n}, T-a_{n}\right]}\left|Z_{n}(t)\right| \leq \max _{1 \leq j \leq N}\left|Z_{n}\left(s_{j}^{n}\right)\right|+w\left(Z_{n} ; n^{-1}\right) .
\end{aligned}
$$

In particular, we have

$$
P\left(\sup _{t \in\left[a_{n}, T-a_{n}\right]}\left|F_{n}(t)\right| \in A^{c_{1} v_{n}+\varepsilon}\right) \leq P\left(\max _{1 \leq j \leq N}\left|F_{n}\left(s_{j}^{n}\right)\right| \in A^{c_{1} v_{n}+2 \varepsilon}\right)+P\left(w\left(F_{n} ; n^{-1}\right)>\varepsilon\right) .
$$

Let us denote by $\kappa_{4}(Y)$ the fourth cumulant of a random variable $Y$ if it exists. Then we have

$$
\kappa_{4}\left(F_{n}(t)\right)=\frac{1}{\mathfrak{s}_{n}^{4}(t)} \sum_{i=1}^{n} K_{h}\left(t_{i-1}-t\right)^{4} \kappa_{4}\left(\left(B_{t_{i}}-B_{t_{i-1}}\right)^{2}\right) \lesssim(n h)^{2} \cdot \frac{n h}{h^{4}} \cdot \frac{1}{n^{4}}=\frac{1}{n h},
$$

hence Lemmas 2.1-2.2 imply that there is a constant $c_{0}>0$ such that

$$
P\left(\max _{1 \leq j \leq N}\left|F_{n}\left(s_{j}^{n}\right)\right| \in A^{c_{1} v_{n}+2 \varepsilon}\right) \leq P\left(\max _{1 \leq j \leq N}\left|Z_{n}\left(s_{j}^{n}\right)\right| \in A^{c_{1} v_{n}+7 \varepsilon}\right)+\varepsilon^{-2} \frac{c_{0}}{\sqrt{n h}} \log ^{2} N .
$$

Since we have $\log N \lesssim \log n$ and

$$
P\left(\max _{1 \leq j \leq N}\left|Z_{n}\left(s_{j}^{n}\right)\right| \in A^{c_{1} v_{n}+7 \varepsilon}\right) \leq P\left(\sup _{t \in\left[a_{n}, T-a_{n}\right]}\left|Z_{n}(t)\right| \in A^{c_{1} v_{n}+8 \varepsilon}\right)+P\left(w\left(Z_{n} ; n^{-1}\right)>\varepsilon\right),
$$

we obtain the desired result. 
Proof of Proposition 4.3. We first prove the second assertion (hence we assume [SB1]). Set $\varepsilon=(n h)^{-\frac{1}{6}} \sqrt{\log n}$. Lemmas B.17 and A.3 imply that there are constants $c_{1}, c_{2}>0$ such that

$$
\begin{aligned}
& \sup _{x \in \mathbb{R}}\left|P\left(\sup _{t \in\left[a_{n}, T-a_{n}\right]}\left|\frac{\widehat{\sigma}_{n}^{2}(t)-\sigma^{2}(t)}{\sigma^{2}(t) \mathfrak{s}_{n}(t)}\right| \leq x\right)-P\left(\sup _{t \in\left[a_{n}, T-a_{n}\right]}\left|Z_{n}(t)\right| \leq x\right)\right| \\
& \leq \sup _{x \in \mathbb{R}} P\left(\left|\sup _{t \in\left[a_{n}, T-a_{n}\right]}\right| Z_{n}(t)|-x| \leq c_{1} v_{n}+8 \varepsilon\right)+\varepsilon^{-2} \frac{c_{2}}{\sqrt{n h}} \log ^{2} n \\
& \quad+P\left(e_{n}>\varepsilon\right)+P\left(w\left(F_{n} ; n^{-1}\right)>\varepsilon\right)+P\left(w\left(Z_{n} ; n^{-1}\right)>\varepsilon\right)
\end{aligned}
$$

for any Borel set $A$ of $\mathbb{R}$ and any $n \in \mathbb{N}$. By definition we have

$$
\varepsilon^{-2} \frac{c_{2}}{\sqrt{n h}} \log ^{2} n=O\left(\frac{\log n}{(n h)^{1 / 6}}\right) .
$$

Also, noting $\log ^{6} n / n h \rightarrow 0$ by assumption, we have $\varepsilon^{-1} h^{\gamma} \log ^{2} n=\sqrt{n h \log n} h^{\gamma} \cdot \log n /(n h)^{1 / 3} \lesssim 1 / \log n$ and $\varepsilon^{-1} \sqrt{\log n} / n h=(n h)^{-5 / 6} \lesssim 1 / \log n$ Therefore, Lemma B.14, (B.8) and the Markov inequality implies that $P\left(e_{n}>\varepsilon\right)=O\left(n^{-1}\right)$ and $P\left(w\left(Z_{n} ; n^{-1}\right)>\varepsilon\right)=O\left(n^{-1}\right)$. Moreover, Lemma B.16 yields

$$
\lim _{n \rightarrow \infty} \sup _{x \in \mathbb{R}} P\left(\left|\sup _{t \in\left[a_{n}, T-a_{n}\right]}\right| Z_{n}(t)|-x| \leq c_{1} v_{n}+8 \varepsilon\right)=O\left(v_{n} \sqrt{\log n}\right)+O\left(\frac{\log n}{(n h)^{1 / 6}}\right) .
$$

We also have $\lim _{n \rightarrow \infty} P\left(w\left(F_{n} ; n^{-1}\right)>\varepsilon\right)=O\left(n^{-1}\right)$. In fact, by Proposition A.1 there is a universal constant $M>0$ such that the process $\left(F_{n}(t)\right)_{t \in[0, T]}$ is a sub-2nd-Gaussian chaos process with respect to the semi-metric $\mathfrak{d}_{n}$ defined by $\mathfrak{d}_{n}(s, t)=M \sqrt{E\left[\left|F_{n}(s)-F_{n}(t)\right|^{2}\right]}, s, t \in[0, T]$. Hence Proposition A.3 and Lemma B.15 imply that

$$
\begin{aligned}
\left\|w\left(F_{n} ; n^{-1}\right)\right\|_{\psi_{1}} & \leq\left\|\sup _{s, t \in[0, T]: \mathfrak{o}_{n}(s, t) \leq M \sqrt{C} / n h}\left|F_{n}(s)-F_{n}(t)\right|\right\|_{\psi_{1}} \\
& \lesssim \int_{0}^{M \sqrt{C} / n h} \log \left(N\left([0, T], \mathfrak{d}_{n}, \eta\right)\right) d \eta \lesssim \int_{0}^{M \sqrt{C} / n h} \log (\eta h)^{-1} d \eta \lesssim \frac{\log n}{n h},
\end{aligned}
$$

where $C$ denotes the constant appearing in Lemma B.15. Hence the desired result follows from the Markov inequality and the fact that $\varepsilon^{-1} \log n / n h=(n h)^{-5 / 6} \sqrt{\log n} \lesssim 1 / \log n$. Overall, we obtain the desired result because $n^{-1}=o\left(\log n /(n h)^{1 / 6}\right)$.

Next we prove the first assertion. For each $k \in \mathbb{N}$, let us define the stopping time $\tau_{k}$ by

$$
\tau_{k}=\inf \left\{t \in[0, T]:|\sigma(t)|>k \text { or } \frac{1}{|\sigma(t)|}>k \text { or } \sup _{\eta \in(0,1)} \eta^{-\gamma} w(\sigma ; \eta, t)>k\right\},
$$

where $w(\sigma ; \eta, t)=\sup \{|\sigma(s)-\sigma(r)|: 0 \leq s, r \in t,|s-r| \leq \eta\}$. Then we define the process $\sigma_{k}=\left(\sigma_{k}(t)\right)_{t \in[0, T]}$ by $\sigma_{k}(t)=\sigma\left(t \wedge \tau_{k}\right), t \in[0, T]$. By construction $\sigma_{k}$ satisfies [SB1], hence we have

$$
\sup _{x \in \mathbb{R}}\left|P\left(\sup _{t \in\left[a_{n}, T-a_{n}\right]}\left|\frac{\widehat{\sigma}_{k, n}^{2}(t)-\sigma_{k}^{2}(t)}{\sigma_{k}^{2}(t) \mathfrak{s}_{n}(t)}\right| \leq x\right)-P\left(\sup _{t \in\left[a_{n}, T-a_{n}\right]}\left|Z_{n}(t)\right| \leq x\right)\right| \rightarrow 0
$$

as $n \rightarrow \infty$ by the second assertion proved above, where

$$
\widehat{\sigma}_{k, n}^{2}(t)=\sum_{i=1}^{n} K_{h}\left(t_{i-1}-t\right)\left(\int_{t_{i-1}}^{t_{i}} \sigma_{k}(s) d B_{s}\right)^{2}
$$


Now, since we have

$$
\sup _{x \in \mathbb{R}}\left|P\left(\sup _{t \in\left[a_{n}, T-a_{n}\right]}\left|\frac{\widehat{\sigma}_{k, n}^{2}(t)-\sigma_{k}^{2}(t)}{\sigma_{k}^{2}(t) \mathfrak{s}_{n}(t)}\right| \leq x\right)-P\left(\sup _{t \in\left[a_{n}, T-a_{n}\right]}\left|\frac{\widehat{\sigma}_{n}^{2}(t)-\sigma^{2}(t)}{\sigma^{2}(t) \mathfrak{s}_{n}(t)}\right| \leq x\right)\right| \leq 2 P\left(\tau_{k}>T\right),
$$

we conclude that

$$
\limsup _{n \rightarrow \infty} \sup _{x \in \mathbb{R}}\left|P\left(\sup _{t \in\left[a_{n}, T-a_{n}\right]}\left|\frac{\widehat{\sigma}_{k, n}^{2}(t)-\sigma_{k}^{2}(t)}{\sigma_{k}^{2}(t) \mathfrak{s}_{n}(t)}\right| \leq x\right)-P\left(\sup _{t \in\left[a_{n}, T-a_{n}\right]}\left|Z_{n}(t)\right| \leq x\right)\right| \leq 2 P\left(\tau_{k}>T\right)
$$

for all $k$. Letting $k \rightarrow \infty$, we obtain the desired result because $P\left(\tau_{k}>T\right) \rightarrow 0$ by [B1].

\section{Acknowledgements}

The author thanks Kengo Kato and Daisuke Kurisu for their helpful comments. The author is also grateful to the Editor and two anonymous referees for their constructive comments which have significantly improved the original version of the paper.

\section{References}

[1] Aït-Sahalia, Y. and Jacod, J. (2014). High-frequency financial econometrics. Princeton University Press.

[2] Alvarez, A., Panloup, F., Pontier, M. and Savy, N. (2012). Estimation of the instantaneous volatility. Stat. Inference Stoch. Process. 15, 27-59.

[3] Andersen, T. G., Bollerslev, T. and Dobrev, D. (2007). No-arbitrage semi-martingale restrictions for continuous-time volatility models subject to leverage effects, jumps and i.i.d. noise: Theory and testable distributional implications. $J$. Econometrics 138, 125-180.

[4] Barlow, M. T. and Yor, M. (1982). Semi-martingale inequalities via the Garsia-Rodemich-Rumsey lemma and application to local times. J. Funct. Anal. 49, 198-229.

[5] Bennedsen, M., Hounyo, U., Lunde, A. and Pakkanen, M. S. (2016). The local fractional bootstrap. Working paper. Available at arXiv: https://arxiv.org/abs/1605.00868.

[6] Bibinger, M., Jirak, M. and Vetter, M. (2017). Nonparametric change-point analysis of volatility. Ann. Statist. 45, $1542-1578$.

[7] Bühlmann, P. and van de Geer, S. (2011). Statistics for high-dimensional data. Springer.

[8] Buldygin, V. V. and Kozachenko, Y. V. (1981). Sub-gaussian random variables. Ukrainian Math. J. 32, 483-489.

[9] Chang, J., Qiu, Y., Yao, Q. and Zou, T. (2017). On the statistical inference for large precision matrices with dependent data. Working paper. Available at arXiv: https://arxiv.org/abs/1603.06663.

[10] Chen, X. (2018). Gaussian and bootstrap approximations for high-dimensional U-statistics and their applications. Ann. Statist. 46, 642-678.

[11] Chernozhukov, V., Chetverikov, D. and Kato, K. (2013). Gaussian approximations and multiplier bootstrap for maxima of sums of high-dimensional random vectors. Ann. Statist. 41, 2786-2819.

[12] Chernozhukov, V., Chetverikov, D. and Kato, K. (2014). Anti-concentration and honest, adaptive confidence bands. Ann. Statist. 42, 1787-1818.

[13] Chernozhukov, V., Chetverikov, D. and Kato, K. (2014). Gaussian approximation of suprema of empirical processes. Ann. Statist. 42, 1564-1597.

[14] Chernozhukov, V., Chetverikov, D. and Kato, K. (2014). Testing many moment inequalities. Working paper. Available at arXiv: https://arxiv.org/abs/1312.7614v4. 
[15] Chernozhukov, V., Chetverikov, D. and Kato, K. (2015). Comparison and anti-concentration bounds for maxima of Gaussian random vectors. Probab. Theory Related Fields 162, 47-70.

[16] Chernozhukov, V., Chetverikov, D. and Kato, K. (2016). Empirical and multiplier bootstraps for suprema of empirical processes of increasing complexity, and related Gaussian couplings. Stochastic Process. Appl. 126, 3632-3651.

[17] Chernozhukov, V., Chetverikov, D. and Kato, K. (2017). Central limit theorems and bootstrap in high dimensions. Ann. Probab. 45, 2309-2353.

[18] Christensen, K., Oomen, R. and Renò, R. (2016). The drift burst hypothesis. Working paper. Available at SSRN: https://ssrn.com/abstract=2842535.

[19] Dalalyan, A. and Yoshida, N. (2011). Second-order asymptotic expansion for a non-synchronous covariation estimator. Ann. Inst. Henri Poincaré Probab. Stat. 47, 748-789.

[20] Davidson, R. and Flachaire, E. (2008). The wild bootstrap, tamed at last. J. Econometrics 146, 162-169.

[21] Davidson, R. and MacKinnon, J. G. (1999). The size distortion of bootstrap tests. Econometric Theory 15, 361-376.

[22] Dirksen, S. (2015). Tail bounds via generic chaining. Electron. J. Probab. 20, 1-29.

[23] Dudley, R. M. (2002). Real analysis and probability. Cambridge University Press.

[24] Fan, J. and Wang, Y. (2008). Spot volatility estimation for high-frequency data. Stat. Interface 1, 279-288.

[25] Götze, F. and Tikhomirov, A. N. (1999). Asymptotic distribution of quadratic forms. Ann. Probab. 27, 1072-1098.

[26] Hayashi, T. and Koike, Y. (2018). Wavelet-based methods for high-frequency lead-lag analysis. SIAM J. Financial Math. 9, 1208-1248.

[27] Hayashi, T. and Yoshida, N. (2005). On covariance estimation of non-synchronously observed diffusion processes. Bernoulli 11, 359-379.

[28] Hayashi, T. and Yoshida, N. (2008). Asymptotic normality of a covariance estimator for nonsynchronously observed diffusion processes. Ann. Inst. Statist. Math. 60,357-396.

[29] Hayashi, T. and Yoshida, N. (2011). Nonsynchronous covariation process and limit theorems. Stochastic Process. Appl. 121, 2416-2454.

[30] Hoffmann, M., Rosenbaum, M. and Yoshida, N. (2013). Estimation of the lead-lag parameter from non-synchronous data. Bernoulli 19, 426-461.

[31] Horn, R. A. and Johnson, C. R. (2013). Matrix analysis. Cambridge University Press, 2nd edn.

[32] Janson, S. (1997). Gaussian Hilbert space. Cambridge University Press.

[33] Kanaya, S. (2017). Uniform convergence rates of kernel-based nonparametric estimators for continuous time diffusion processes: A damping function approach. Econometric Theory 33, 874-914.

[34] Kanaya, S. and Kristensen, D. (2016). Estimation of stochastic volatility models by nonparametric filtering. Econometric Theory 32, 861-916.

[35] Kato, K. and Kurisu, D. (2017). Bootstrap confidence bands for spectral estimation of Lévy densities under highfrequency observations. Working paper. Available at arXiv: https://arxiv.org/abs/1705.00586.

[36] Kato, K. and Sasaki, Y. (2016). Uniform confidence bands in deconvolution with unknown error distribution. Working paper. Available at arXiv: https://arxiv.org/abs/1608.02251.

[37] Krakowiak, W. and Szulga, J. (1988). Hypercontraction principle and random multilinear forms. Probab. Theory Related Fields 77, 325-342.

[38] Kristensen, D. (2010). Nonparametric filtering of the realized spot volatility: A kernel-based approach. Econometric Theory 26, 60-93. 
[39] Kusuoka, S. and Yoshida, N. (2000). Malliavin calculus, geometric mixing, and expansion of diffusion functionals. Probab. Theory Related Fields 116, 457-484.

[40] Kwapień, S. and Woyczyński, W. A. (1992). Random series and stochastic integrals: Single and multiple. Birkhäuser.

[41] Lee, S. S. and Mykland, P. A. (2008). Jumps in financial markets: A new nonparametric test and jump dynamics. The Review of Financial Studies 21, 2535-2563.

[42] Mancino, M. E. and Recchioni, M. C. (2015). Fourier spot volatility estimator: Asymptotic normality and efficiency with liquid and illiquid high-frequency data. PLOS ONE 10,1-33.

[43] Mossel, E., O'Donnell, R. and Oleszkiewicz, K. (2010). Noise stability of functions with low influences: Invariance and optimality. Ann. Math. 171, 295-341.

[44] Mykland, P. A. and Zhang, L. (2008). Inference for volatility-type objects and implications for hedging. Stat. Interface 1, 255-278.

[45] Nourdin, I. (2013). Lectures on Gaussian approximations with Malliavin calculus. In C. Donati-Martin, A. Lejay and A. Rouault, eds., Séminaire de probabilitiés XLV, vol. 2078 of Lecture Notes in Math. Springer, pp. 3-89.

[46] Nourdin, I. and Peccati, G. (2009). Stein's method on Wiener chaos. Probab. Theory Related Fields 145, 75-118.

[47] Nourdin, I. and Peccati, G. (2010). Stein's method meets Malliavin calculus: a short survey with new estimates. In J. Duan, S. Luo and C. Wang, eds., Recent development in stochastic dynamics and stochastic analysis, chap. 14. World Scientific, pp. 207-236.

[48] Nourdin, I. and Peccati, G. (2012). Normal approximations with Malliavin calculus: From Stein's method to universality. Cambridge University Press.

[49] Nourdin, I., Peccati, G. and Reinert, G. (2009). Second order Poincaré inequalities and CLTs on Wiener space. J. Funct. Anal. 257, 593-609.

[50] Nourdin, I., Peccati, G. and Reinert, G. (2010). Invariance principles for homogeneous sums: Universality of Gaussian Wiener chaos. Ann. Probab. 38, 1947-1985.

[51] Nourdin, I., Peccati, G. and Réveillac, A. (2010). Multivariate normal approximation using Stein's method and Malliavin calculus. Ann. Inst. Henri Poincaré Probab. Stat. 46, 45-58.

[52] Nualart, D. (2006). The Malliavin calculus and related topics. Springer, 2nd edn.

[53] Nualart, D. and Peccati, G. (2005). Central limit theorems for sequences of multiple stochastic integrals. Ann. Probab. 33, 177-193.

[54] Palmes, C. and Woerner, J. H. C. (2016). A mathematical analysis of the Gumbel test for jumps in stochastic volatility models. Stoch. Anal. Appl. 34, 852-881.

[55] Rivasplata, O. (2012). Subgaussian random variables: An expository note. Unpublished paper.

[56] Robert, C. Y. and Rosenbaum, M. (2010). On the limiting spectral distribution of the covariance matrices of time-lagged processes. J. Multivariate Anal. 101, 2434-2451.

[57] Sabel, T. (2014). Simultaneous confidence statements about the diffusion coefficient of an Itô-process with application to spot volatility estimation. Ph.D. thesis, der Georg-August University School of Science.

[58] Söhl, J. and Trabs, M. (2016). Adaptive confidence bands for markov chains and diffusions: Estimating the invariant measure and the drift. ESAIM Probab. Stat. 20, 432-462.

[59] van der Vaart, A. W. and Wellner, J. A. (1996). Weak convergence and empirical processes. Springer.

[60] Viens, F. G. and Vizcarra, A. B. (2007). Supremum concentration inequality and modulus of continuity for sub-nth chaos processes. J. Funct. Anal. 248, 1-26. 
[61] Vizcarra, A. B. and Viens, F. G. (2007). Some applications of the Malliavin calculus to sub-Gaussian and non-subGaussian random fields. In R. C. Dalang, F. Russo and M. Dozzi, eds., Seminar on stochastic analysis, random fields and applications V. Progress in probability, vol. 59. Birkhäuser Basel, pp. 363-395.

[62] Zhang, D. and Wu, W. B. (2017). Gaussian approximation for high dimensional time series. Ann. Statist. 45, 1895-1919.

[63] Zhang, X. and Cheng, G. (2018). Gaussian approximation for high dimensional vector under physical dependence. Bernoulli 24, 2640-2675. 